\title{
Electrophysiological signature of conscientiousness during task performance
}

\author{
Inaugural-Dissertation \\ in der Fakultät Humanwissenschaften \\ der Otto-Friedrich-Universität Bamberg
}

vorgelegt von

Mike Frederic Imhof

aus

Siegen

Bamberg, den 03.10.2019 
Tag der mündlichen Prüfung: 10.12.2019

Dekan: $\quad$ Universitätsprofessor Dr. Jörg Wolstein

Erstgutachter: Universitätsprofessor Dr. Jascha Rüsseler

Zweitgutachterin: Universitätsprofessorin Dr. Ute Schmid

URN: urn:nbn:de:bvb:473-irb-478631

DOI: https://doi.org/10.20378/irb-47863

Dieses Werk ist als freie Onlineversion über das Forschungsinformationssystem (FIS; https://fis.uni-bamberg.de) der Universität Bamberg erreichbar. Das Werk steht unter der CCLizenz CC-BY.

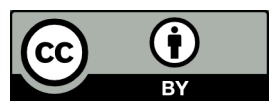


To Nadine, Selma and Linus 


\section{Zusammenfassung}

Die Forschung zur biologischen Basis der Persönlichkeit hat eine lange Tradition. Obwohl das populäre und weit verbreitete Fünf-Faktoren-Modell (FFM) der Persönlichkeit nicht biologisch basiert ist gibt es Hinweise darauf, dass Unterschiede in Gehirnstrukturen mit individuellen Unterschieden in Persönlichkeitsfaktoren des FFM zusammenhängen. Es gibt jedoch bisher nur wenige Untersuchungen zu Unterschieden in der aufgabenbezogenen Gehirnaktivität, die mit Unterschieden in Persönlichkeitsfaktoren des FFM wie beispielsweise der Gewissenhaftigkeit verbunden sind. Das ist überraschend, denn die Untersuchung von Unterschieden in ereigniskorrelierten Potenzialen (EKP) im Zusammenhang mit Gewissenhaftigkeit scheint ein vielversprechender Ansatz zu sein. Das zeigen z. B. Hinweise darauf, dass Gewissenhaftigkeit mit der Error-related negativity (ERN) zusammenhängt.

Kognitiven Theorien zufolge sollte die Größe der ERN mit behavioralen Unterschieden zusammenhängen. Aktuelle Forschungen deuten jedoch darauf hin, dass die ERN und behaviorale Maße zumindest teilweise unabhängig voneinander sind. Persönlichkeitseigenschaften, psychopathologische und motivationale Faktoren scheinen Mechanismen der Generierung der ERN zu beeinflussen und so die Amplitude der ERN zu moderieren. Als Gegenstück zur ERN tritt die Correct-response negativity (CRN) nach korrekten Antworten auf. Ausgehend von der Annahme, dass die CRN zumindest teilweise den gleichen kognitiven Prozess widerspiegelt, sollte auch die Amplitude der CRN durch Persönlichkeitsmerkmale und motivationale Faktoren moderiert werden.

Ich habe zwei Experimente durchgeführt, um die Auswirkungen der Gewissenhaftigkeit auf das aufgabenbezogene EKP nach Fehlern und richtigen Reaktionen zu untersuchen. In beiden Experimenten habe ich mithilfe von einfachen Wahlreaktionsaufgaben in unterschiedlichen Varianten die Amplituden der ERN und CRN im Zusammenhang mit Gewissenhaftigkeit und absichtlichen Regelverletzungen untersucht. Ich beobachtete Un- 
terschiede in der Amplitude der $\triangle E R N$ (der Differenzamplitude von ERN und CRN) im Zusammenhang mit Gewissenhaftigkeit, die auf eine stärkere Fokussierung auf die Aufgabe und eine höhere Motivation richtig zu reagieren bei hoch gewissenhaften Personen hinweisen könnten. Zudem beobachtete ich Unterschiede in der CRN-Amplitude, die auf einen durch Gewissenhaftigkeit moderierten Responsekonflikt zurückzuführen sind. Die Ergebnisse zeigen einen Zusammenhang von Gewissenhaftigkeit und dem aufgabenbezogenen, reaktionsbezogenen EKP.

Schließlich habe ich untersucht, ob Unterschiede im EKP als Biomarker für die Klassifikation von gewissenhaften Personen durch die Anwendung der linearen Diskriminanzanalyse (LDA) und der linearen Regression (LR) verwendet werden können. LDA und LR sind populäre Methoden im Bereich des maschinellen Lernens, die für die Klassifikation von single-trial und aggregierten EKP-Daten geeignet sind. Ich schlage ein Klassifikationsverfahren vor, das auch für die Klassifikation anderer Merkmale, die mit EKP-Daten zusammenhängen, verwendet werden kann. Sowohl LDA als auch LR, die in das Verfahren eingebettet sind, zeigen ihre Eignung und Nützlichkeit, indem sie die EKP-Datensätze beider Experimente neu analysieren. Die Klassifikationsperformanz wurde mittels Kreuzvalidierung evaluiert und zeigte, dass beide Methoden erfolgreich zwischen zwei Gruppen von Individuen mit geringer und hoher Gewissenhaftigkeit unterscheiden.

Die zukünftige Forschung sollte sich auf moderierende Effekte auf die Assoziation von Gewissenhaftigkeit und CRN und $\triangle \mathrm{ERN}$ konzentrieren. 


\section{Abstract}

Research on the biological basis of personality has a long tradition. Although the popular and widespread Five Factor Model (FFM) of personality is not biologically based, there is evidence that differences in brain structures are associated with individual differences in personality traits that are part of the FFM. To date, there is only sparse research on differences in task-related brain activity linked with differences in FFM factors such as conscientiousness. This is surprising because the study of differences in event-related potentials (ERPs) in the context of conscientiousness appears to be a promising approach. This is shown, for example, by evidence that conscientiousness is related to the error-related negativity (ERN).

According to cognitive theories the size of the ERN should be related to variations in behavior. However, recent research indicates that the ERN and behavioral measures are at least partly unrelated. It seems evident that individual difference measures, psychopathology, and motivational factors impact mechanisms generating the ERN and therefore moderate the size of the ERN. The CRN is the counterpart of the ERN occurring after correct responses. Based on the assumption that the $\mathrm{CRN}$ is at least partly reflecting an equal cognitive process as the ERN, the size of the $\mathrm{CRN}$ should be moderated by individual difference measures as well.

I conducted two experiments addressing the impact of conscientiousness on the taskrelated ERP after both errors and correct responses. In both experiments, I used simplechoice reaction tasks in different variations to investigate ERN and CRN amplitudes in relation to conscientiousness and deliberate rule violations. I observed variations in the $\triangle \mathrm{ERN}$ amplitude (which is the difference amplitude of ERN and CRN) related to conscientiousness that might indicate a greater focus on the task and a motivational salience of correct responses. I also observed differences in the CRN amplitude that are due to response 


\section{Abstract}

conflict moderated by conscientiousness. The results showed an apparent association of conscientiousness and the task-related response-locked ERP.

Finally, I examined whether differences in the ERP can be used as biomarkers for the classification of conscientious individuals through the application of linear discriminant analysis (LDA) and linear regression (LR), machine learning methods popular for classification of single-trial and aggregated ERP data. I propose a classification procedure that can also be used for classification of other features related to ERP data. Both LDA and LR embedded in the procedure demonstrate their suitability and usefulness by reanalyzing the ERP datasets of both experiments. The classification performance was evaluated using cross validation and showed that both methods successfully discriminated above chance between two groups of individuals scoring low and high on a conscientiousness scale.

Future research should concentrate on moderating effects on the association of conscientiousness and $\mathrm{CRN}$ and $\triangle \mathrm{ERN}$. 


\section{Contents}

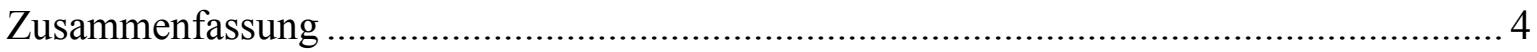

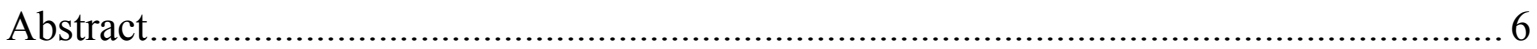

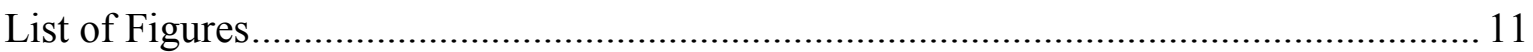

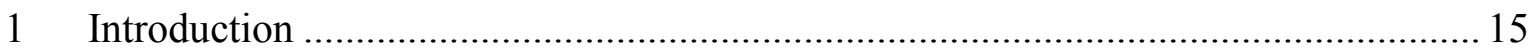

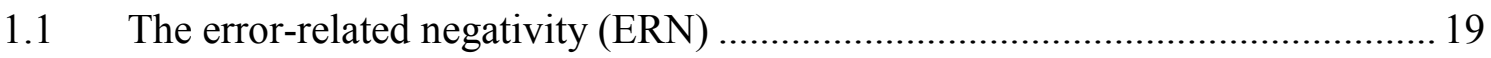

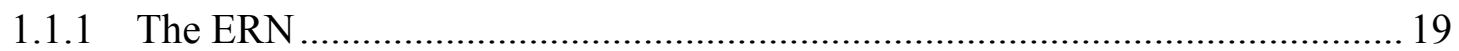

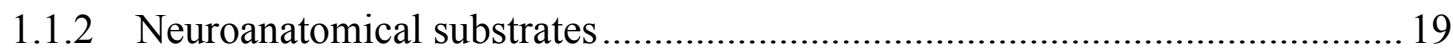

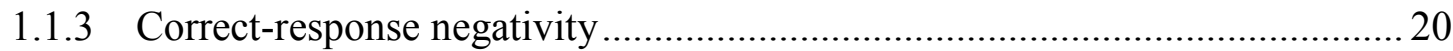

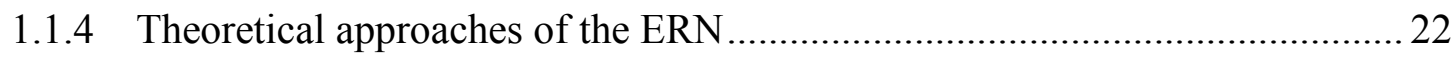

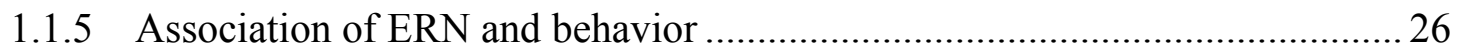

1.1.6 ERN / CRN and individual differences ...................................................... 27

2 Performance monitoring and correct response significance in conscientious

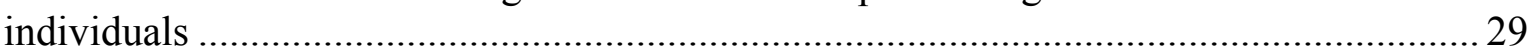

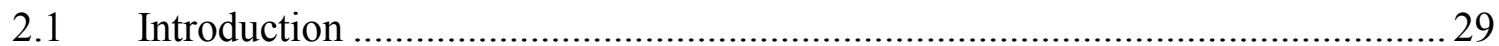

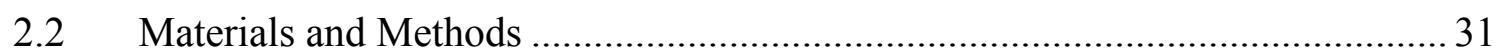

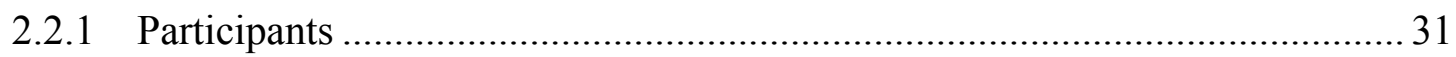

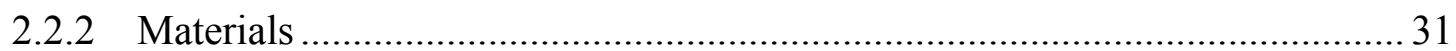

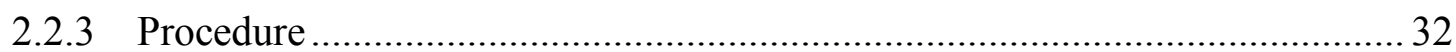

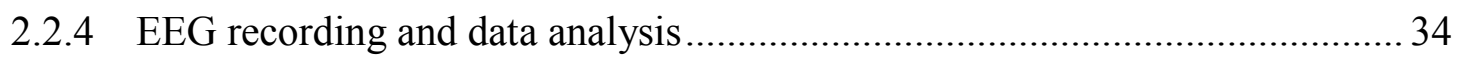

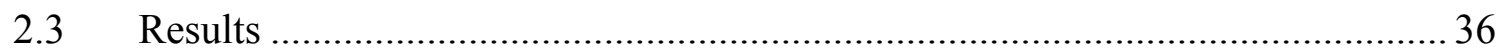

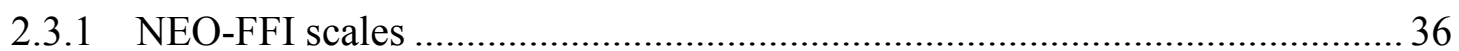

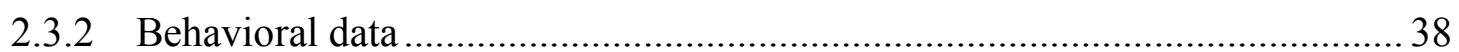

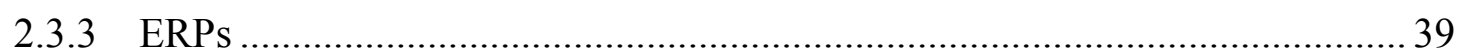

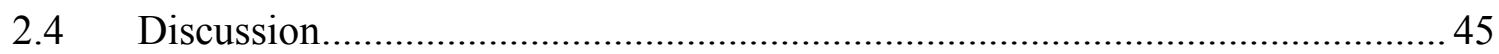

2.4.1 Higher task engagement in conscientious individuals.................................. 46

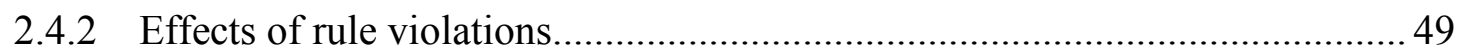

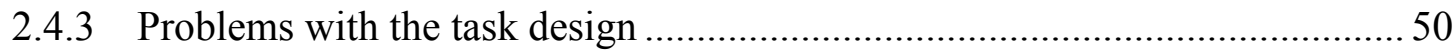




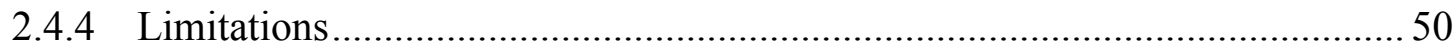

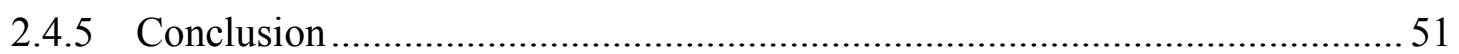

3 The impact of conflict on performance monitoring in conscientious individuals ....... 52

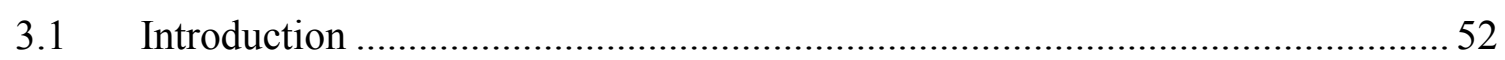

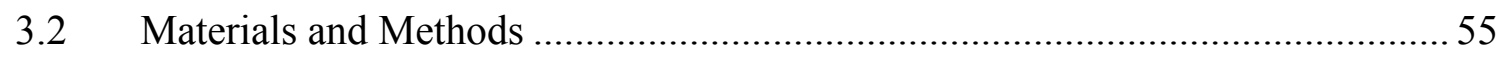

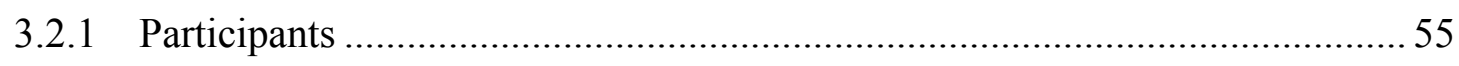

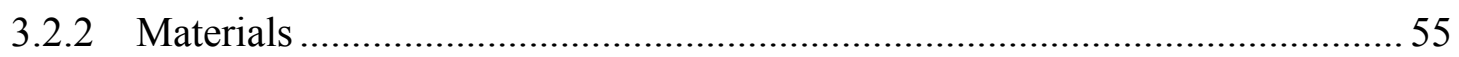

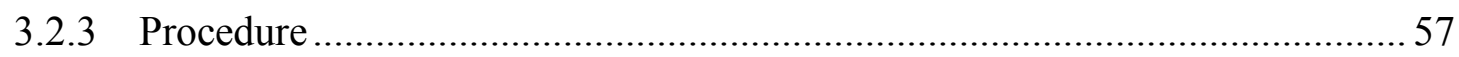

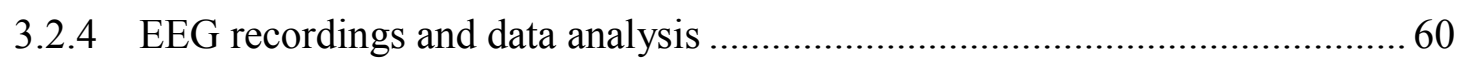

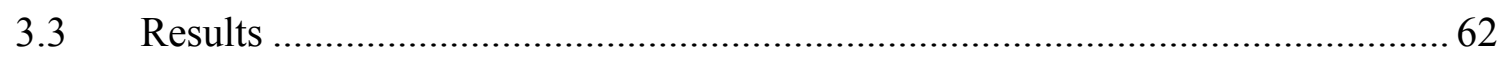

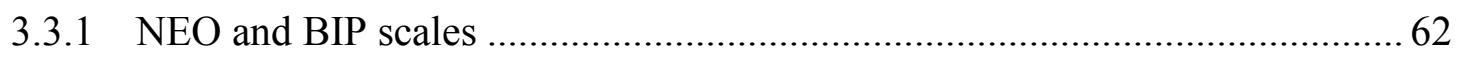

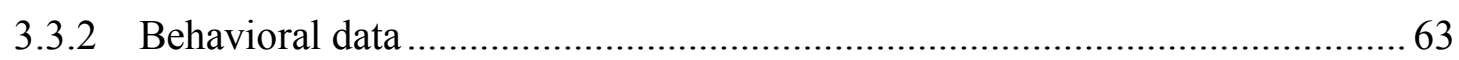

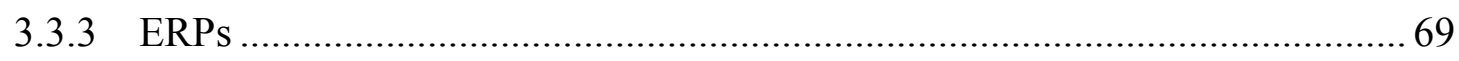

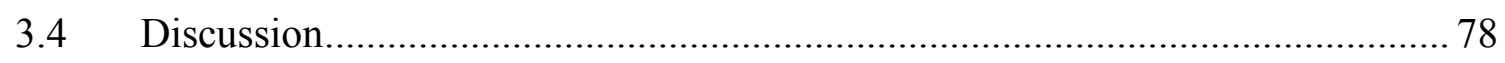

3.4.1 Task engagement in conscientious individuals ............................................ 79

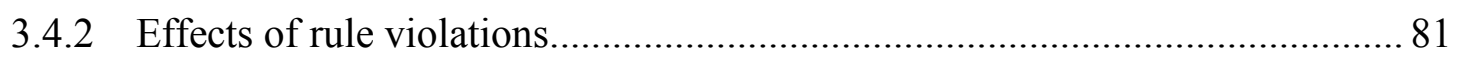

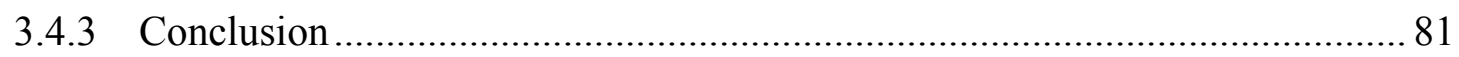

4 Task-related ERPs as biomarkers for classification of conscientiousness ................. 82

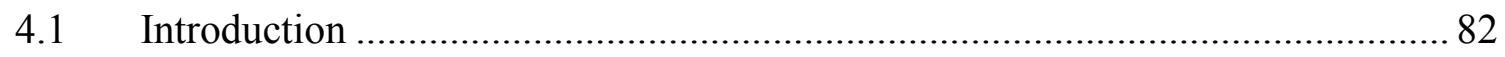

4.2 Approach for classification of conscientiousness using task-related ERPs ........ 85

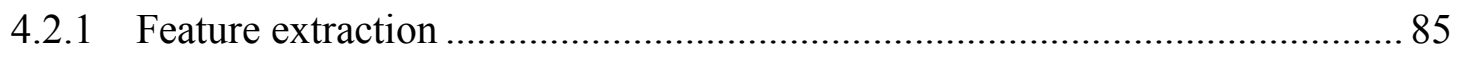

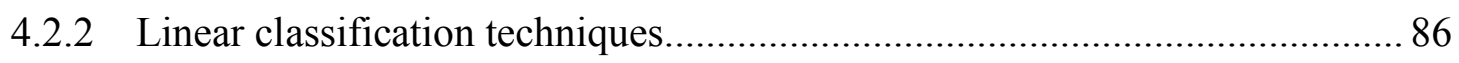

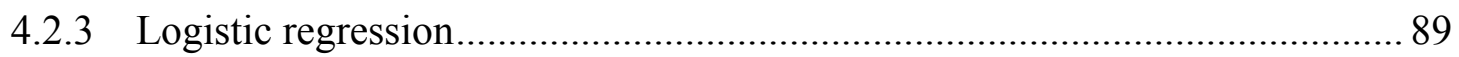

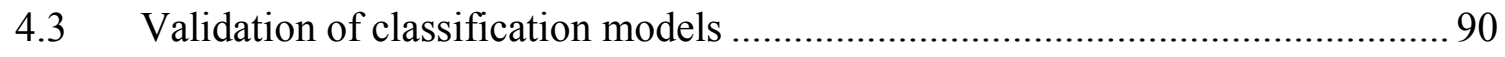

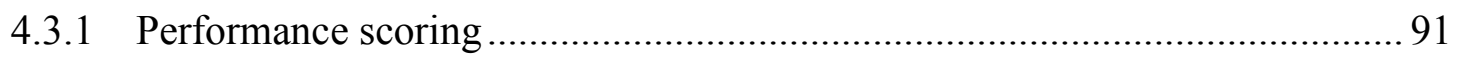

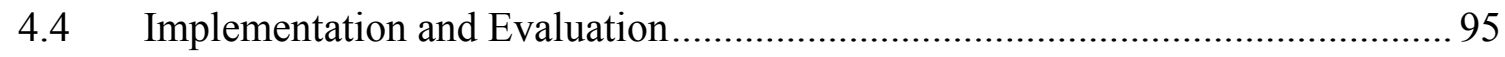

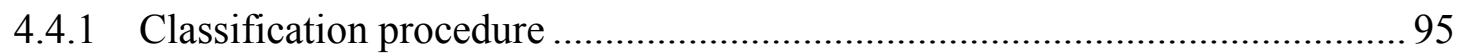

4.4.2 Evaluation using data from Experiment 1 …............................................... 97

4.4.3 Evaluation using data from Experiment 2 ................................................ 102

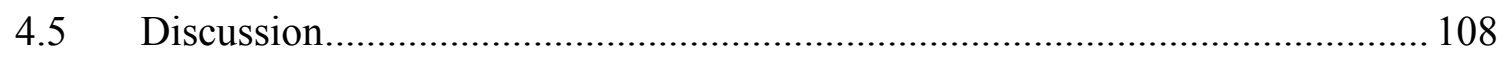

4.5.1 Comparing results with former analyses .................................................. 108

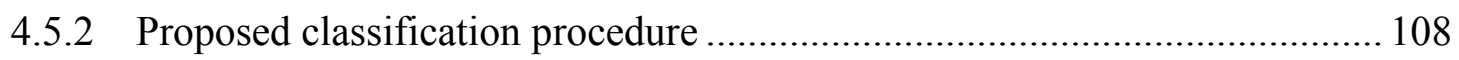




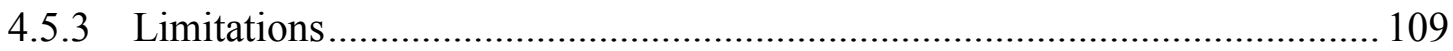

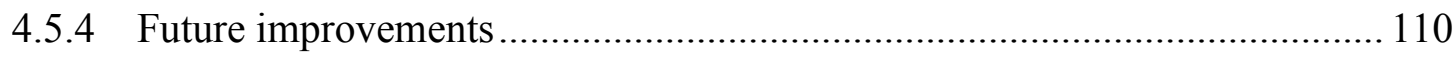

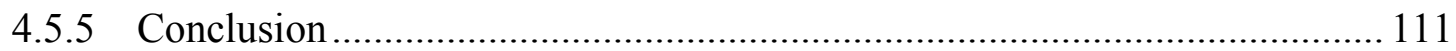

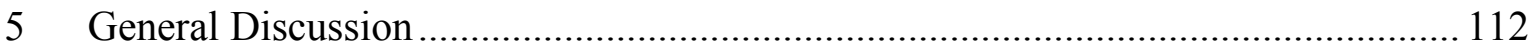

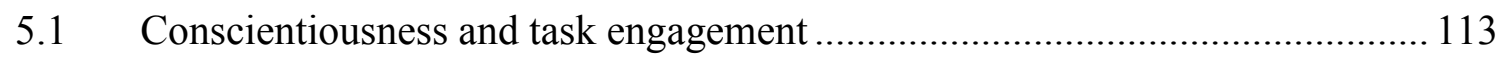

5.2 Classification of conscientiousness using task-related ERPs ........................... 116

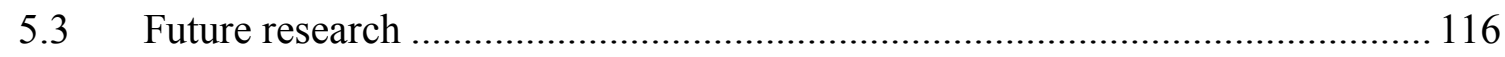

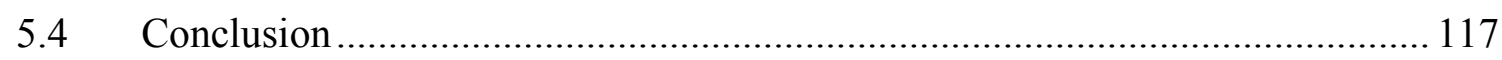

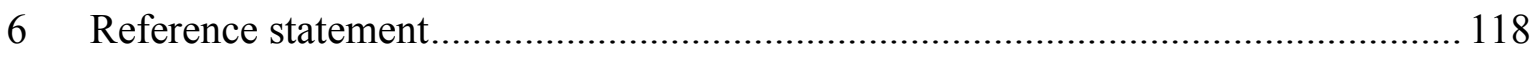

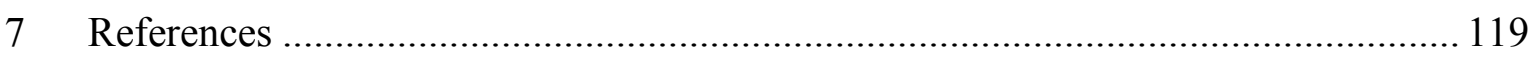




\section{List of Figures}

Figure 1. Illustration of the error detection theory of the ERN proposed by Coles et al. (2001) assuming a comparison process that evaluates the consistency of the representation of the actual response and the representation of the correct response. Inconsistency of both representations elicits a mismatch or error signal which is reflected by the ERN. Adapted from de Bruijn, Miedl, and Bekkering (2011).

Figure 2. Illustration of a conflict-monitoring model for the Eriksen Flanker task. Responses are activated by corresponding stimulus processing units that correspond to the target and flanker triangles $<$ and $>$. Coactivation of the mutually incompatible response units $(<$ and $>$ ) generates the conflict. The presence of conflict is detected by the conflictmonitoring process which then signals adjustments in attention to reduce conflict in the subsequent trials. Adapted from Yeung and Nieuwenhuis (2009). 24

Figure 3. Illustration of the reinforcement-learning theory proposed by Holroyd and Coles (2002). A mechanism in the basal ganglia (BG) monitors the performance of a mechanism located in the anterior cingulate cortex (ACC) that maps external input into response output. An error signal is conveyed to the ACC by the midbrain dopamine system. The theory assumes that the ERN is produced by the ACC. Adapted from Holroyd and Yeung (2003).

Figure 4. Procedure of a trial. After the presentation of a blank screen $(500 \mathrm{~ms})$, the participant is instructed by a short text $(1500 \mathrm{~ms})$. Then the presentation of a fixation cross $(500 \mathrm{~ms})$ follows whereafter the target stimulus is presented until the participant responds.

Figure 5. Distribution of conscientiousness scores obtained from the sample.

Figure 6. Mean error rates (A) and response times (B) for each instruction condition, standard (STD), rule violation (RV), and rule reversal (RR). Scatter plots display the performance as a function of conscientiousness (CS). See text for details. 39

Figure 7. Mean amplitudes during 20-70ms post-response interval at electrode $\mathrm{FCz}$ as a function of conscientiousness for correct (CRN) and incorrect responses (ERN). The lines resulted from regressing $\mathrm{CRN}$ and $\mathrm{ERN}$ amplitudes onto conscientiousness.

Figure 8. Mean amplitudes during 200-350ms post-response interval at electrode $\mathrm{Cz}$ as a function of conscientiousness for correct $(\mathrm{Pc})$ and incorrect responses $(\mathrm{Pe})$. The lines resulted from regressing Pc and Pe amplitudes onto conscientiousness.

Figure 9. Response-locked ERPs for correct responses at electrode $\mathrm{FCz}$ for both conscientiousness (CS) groups separated by instruction conditions. The three panels show the ERPs of standard (STD), rule violation (RV), and rule reversal (RR) conditions, 
respectively. Color-shaded areas indicate the 95\% confidence intervals for the mean ERPs. Gray-shaded areas mark the time windows of CRN and Pc.

Figure 10. Z-transformed voltage distributions during response-locked ERP after correct responses in a time window of 30-50ms. The upper and lower heads show distributions of participants scoring high and low on conscientiousness (CS), respectively. The distributions of each group are displayed separately by column for the instruction conditions standard (STD), rule violation (RV) and rule reversal (RR).

Figure 11. Z-transformed voltage distributions during response-locked ERP after correct responses in a time window of 200-300ms. The upper and lower heads show distributions of participants scoring high and low on conscientiousness (CS), respectively. The distributions of each group are displayed separately by column for the instruction conditions standard (STD), rule violation (RV) and rule reversal (RR).

Figure 12. Procedure of a trial. The following screens were presented in the order they are mentioned including their presentation durations in the following: blank screen (1000ms), instruction $(500 \mathrm{~ms})$, fixation cross $(500 \mathrm{~ms})$, flanker stimuli $(50 \mathrm{~ms})$ and flanker stimuli combined with target stimulus (until response). 58

Figure 13. Distribution of conscientiousness t-scores obtained from sample of Experiment 2.

Figure 14. Mean error rates are displayed separately for each instruction condition, standard (STD), rule violation (RV), and rule reversal (RR) splitted by congruency condition (congruent, incongruent). Scatter plots overlaying each boxplot display the error rate as a function of conscientiousness (CS), the extent of which is represented by color intensity (high CS values are represented by low color intensity).

Figure 15. Mean response times are displayed separately for each instruction condition, standard (STD), rule violation (RV), and rule reversal (RR) splitted by congruency condition (congruent, incongruent). Scatter plots overlaying each boxplot display the response time as a function of conscientiousness (CS), the extent of which is represented by color intensity (high CS values are represented by low color intensity). 66

Figure 16. Mean PES in ms from standard instruction condition is displayed separately for each congruency condition (congruent, incongruent) separated by rule group, rule violation (RV), and rule reversal (RR). Scatter plots overlaying each boxplot display the PES as a function of conscientiousness (CS), the extent of which is represented by color intensity (high CS values are represented by low color intensity). 68

Figure 17. Response-locked ERP at electrode $\mathrm{FCz}$ after errors in incongruent standard trials separated by levels of conscientiousness (CS; low, high). Shaded areas indicate the 95\% confidence interval of the mean ERPs.

Figure 18. Response-locked ERP at electrode FCz following correct responses in standard (STD) instruction condition as well as alternative instruction conditions rule violation (RV) and rule reversal (RR) separated by levels of conscientiousness (CS; low, high) and congruency (congruent, incongruent). For the purpose of illustration low and high conscientiousness groups were formed according to the sample selection. 71 
Figure 19. Z-transformed voltage distributions during response-locked ERP after correct responses in a time window of 10-40ms. The upper and lower heads show distributions of participants scoring high and low on conscientiousness (CS), respectively. The distributions of each group are displayed separately by column for the combinations of instruction conditions, i.e. standard (STD) and alternative (ALT), and congruency type, i.e. congruent and incongruent. 72

Figure 20. Mean amplitude during 0-70ms post-response time interval of the responselocked ERP after correct responses at electrode FCz. The mean amplitudes of individuals scoring low (A) and high (B) on conscientiousness (CS) scale are separately depicted for instruction conditions (STD, ALT) and congruency (congruent, incongruent). Error bars indicate standard error.

Figure 21. Z-transformed voltage distributions during response-locked ERP after correct responses in a time window of $90-110 \mathrm{~ms}$. The upper and lower heads show distributions of participants scoring high and low on conscientiousness (CS), respectively. The distributions of each group are displayed separately by column for the combinations of instruction conditions, i.e. standard (STD) and alternative (ALT), and congruency type, i.e. congruent and incongruent. 75

Figure 22. Mean amplitude during $80-150 \mathrm{~ms}$ post-response time interval of the responselocked ERP after correct responses at electrode $\mathrm{Cz}$. The mean amplitudes of individuals scoring low (A) and high (B) on conscientiousness (CS) scale are separately depicted for instruction conditions (STD, ALT) and congruency (congruent, incongruent). Error bars indicate standard error.

Figure 23. Exemplary visualization of spatio-temporal features that consist of a matrix with signed- $r^{2}$ values of low- minus high-CS averaged ERPs. The matrix is displayed as color coded matrix.

Figure 24. A schematical illustration of the classification procedure that was used for ERP data from Experiment 1. The experiment consisted of a choice reaction task where participants during standard (STD) condition had to respond to a circle by pressing the left key and to a square by pressing the right key. In two other conditions participants had to either violate $(R V)$ or reverse this rule (RR). EEG was recorded throughout the experiment. In this illustration only the electrode $\mathrm{FCz}$ is shown (A). Other electrodes are schematically indicated by different superimposed levels. The continuous signal was split into response-locked epochs (B). The raw EEG from electrode $\mathrm{FCz}$ is shown over a period of $8 \mathrm{~s}$, the rectangles show a $800 \mathrm{~ms}$ epoch, beginning $100 \mathrm{~ms}$ before the response. Separate averages were then computed for the three different segment types (C). After that Pearson r-values of conscientiousness and averaged response-locked ERPs were calculated for the spatio-temporal features and were displayed as color coded matrix (D). The matrix was used to determine time windows for averaging the voltage values for each electrode (E). The averaged spatio-temporal features were finally used for classification learning (F). .. 94

Figure 25. Visualization of the r-matrices. Pearson r-values of conscientiousness and averaged response-locked ERPs were calculated for the spatio-temporal features and displayed as a color coded matrix for each instruction condition (A: Standard, B: Rule violation, C: Rule reversal) of Experiment 1 . Time intervals that are indicated by a dashed line were selected by application of a heuristic that aims to ensure having accumulated 
high r-values and an approximately constant spatial pattern of r-values in each time interval. The areas that are bounded by a continuous line indicate significant $\mathrm{r}$-values $(p<$ .05 ) and help to identify suitable time intervals for classification learning.

Figure 26. Performance as mean accuracy of linear discriminant analysis (LDA) with shrinkage of the covariance matrix and logistic regression (LR) for each instruction condition standard (STD), rule violation (RV) and rule reversal (RR) of Experiment 1. Mean accuracy values are aggregated for 10 iterations of 6 -fold cross validations. Error bars indicate the standard deviation.

Figure 27. Performance as receiver operating characteristics (ROCs) of linear discriminant analysis (LDA) with shrinkage of the covariance matrix and logistic regression (LR) for each instruction condition standard (STD), rule violation (RV) and rule reversal (RR) of Experiment 1. ROC curves are displayed and area under the curve (AUC) values are calculated for each iteration step. 101

Figure 28. Visualization of the r-matrices. Pearson r-values of conscientiousness and averaged response-locked ERPs were calculated for the spatio-temporal features and were displayed as a color coded matrix for each combination of instruction and congruency condition (A: Standard congruent, B: Standard incongruent, C: Alternative instruction congruent, D: Alternative instruction incongruent) of Experiment 2 (Note: Rule violation and rule reversal instruction condition were taken together because there were no significant differences between both conditions). Time intervals that are indicated by a dashed line were selected by application of a heuristic that aims at ensuring to have accumulated high r-values and an approximately constant spatial pattern of r-values in each time interval. The areas that are bounded by a continuous line indicate significant $r$-values $(p<.05)$ and help to identify suitable time intervals for classification learning. 103

Figure 29. Performance of linear discriminant analysis (LDA) with shrinkage of the covariance matrix and logistic regression (LR) for each combination of instruction condition (standard, STD; alternative instruction, ALT) and congruency condition (congruent, incongruent) of Experiment 2. Mean accuracy values are aggregated for 10 iterations of 6-fold cross validations. Error bars indicate the standard deviation. 105

Figure 30. Performance as receiver operating characteristics (ROCs) of linear discriminant analysis (LDA) with shrinkage of the covariance matrix and logistic regression (LR) for each combination of instruction condition (standard, STD; alternative instruction, ALT) and congruency condition (congruent, incongruent) of Experiment 2. ROC curves are displayed and area under the curve (AUC) values are calculated for each iteration step. 106 


\section{Introduction}

The notion that individual differences in personality have a physiological basis has a long tradition. Philosophers of ancient Greece supposed that personality was influenced by various fluids, so-called humors, in the body. According to the Greek physicians Hippocrates and Galen, there were four main humors, each contributing to the constitution of personality and, depending on their distribution, emphasizing a particular pattern of personality. This idea remained popular during medieval times, and even influential in modern research. The famous Russian psychologist and physiologist Ivan Pavlov suggested that there were four basic kinds of temperament which he believed to have observed in dogs. Although he never tried to study the temperament types in humans, he believed that there were parallels between humans and dogs and that the temperament types observed in dogs also applied to humans. In recent times, researchers have tried to study Pavlovs temperament types in humans developing self-report questionnaire scales for this purpose (Strelau, Angleitner, Bantelmann, \& Ruch, 1990). Their results indicated similarities between characteristics of Pavlov's temperament types and characteristics associated with the four humors (Ruch, 1992). However, the results are rather descriptive and do not allow indications for biological causes of individual differences.

Modern approaches try to fill this lack of understanding and focus on the biological causes of individual differences. Many researchers have focused on neurotransmitters (e.g. Cloninger, 1987; Cloninger, Przybeck, Svrakic, \& Wetzel, 1994). For instance, according to Cloninger's theory, high levels of dopamine are associated with high levels of novelty seeking, high levels of serotonin are associated with low levels of harm avoidance, and high levels of norepinephrine are associated with low levels of reward dependence. Other influential modern approaches on biological causes of individual differences examine various structures in the brain. Some researchers have suggested that individual differences in personality are linked to complex interactions among different regions of the brain. 
A famous approach is the arousal hypothesis of Eysenck (1967, 1990). Central to Eysenck's theory is the ascending reticular activating system (ARAS). According to this approach, this system is located in the brain stem, where the spinal cord meets the brain itself. It works like a filter to regulate the level of arousal in the brain and hence ensures an effective functioning of the individual. There are certain conditions that increase the arousal, e.g. when an individual is excited by listening to an activating piece of music or concentrating intensely on solving a difficult task. And there are other conditions under which the level of arousal is reduced, e.g. at bedtime when an individual is trying to fall asleep. Eysenck (1967) proposed that introverts have higher levels of activity or a lower threshold of arousal in the ARAS than extraverts. Following this arousal hypothesis, it is due to physiological processes regulated by the ARAS that introverts react with greater responsiveness than extraverts. Because introverts experience more arousal due to higher level of ARAS activity, they should react earlier and to a greater extent than extraverts to various forms of external stimulation. In line with this perspective, studies show that introverts seek for situations with low stimulation to keep their already heightened level of ARAS activity in balance. In contrast, extraverts need to increase their already low level of arousal (Beauducel, Brocke, \& Leue, 2006; Claridge, Donald, \& Birchall, 1981). There is evidence that supports the arousal hypothesis proposing a relationship between introversion/extraversion and the ARAS (Geen, 1984; for a review see Eysenck, 1990; Matthews \& Gilliland, 1999; Stelmack, 1990), including studies where the cortical arousal is measured by electroencephalogram (EEG; Knyazev et al., 2002) and positron emission tomography (Johnson et al., 1999).

Another influential theory describing brain structures that are associated with individual differences in personality was proposed by Gray $(1972,1990)$ called reinforcement sensitivity theory. On an abstract level, the theory assumes two biological systems in the brain: The first system is the Behavioral Approach System (BAS) which is responsive to rewarding stimuli. As soon as the BAS recognizes a stimulus as potentially rewarding, approaching behavior is activated. For example, when you hear your favorite song on the radio, the BAS causes increased attention to the song and it may create the urge to turn up the radio (approach motivation). The other system is the Behavioral Inhibition System 
(BIS). When activated, it increases the sensitivity to punishing stimuli and regulates behavior for avoidance of the stimuli. For example, when confronted with an unpleasant situation that you fear, the BIS causes the internal creation of a plan to avoid confronting with this situation. According to the reinforcement sensitivity theory, individuals differ in the sensitivity of their BIS or BAS system. Individuals with a reactive BIS system have a higher sensitivity to cues of punishment, frustration or novelty, and they are especially vulnerable to unpleasant emotions such as anxiety, fear or sadness. On the other hand, individuals with a reactive BAS system have a higher sensitivity to cues of reward, and they are especially responsive to positive emotions. The ability to inhibit behavior decreases in individuals with a reactive BAS when approaching a stimulus. Following the reinforcement sensitivity theory, the BIS system determines the personality dimension anxiety whereas the BAS system is responsible for the personality dimension of impulsivity.

The models outlined above describe how individual differences in certain personality traits can be explained by biological bases. However, the models do not provide explanations what physiological bases may underlie individual differences in other personality traits that are part of common models of personality, e. g. the Five Factor Model (FFM; Costa \& McCrae, 1992). What also remains unachieved, is a detailed understanding of the physiological systems and mechanisms that influence individual differences in specific personality dimensions. In recent research on personality, biological approaches addressing such mechanisms were most commonly investigated by taking measures of electrodermal activity, cardiovascular reactivity and brain activity obtained from methods like electroencephalogram (EEG) or functional magnetic resonance imaging (fMRI). EEG plays an important role, but investigations of associations with personality traits and individual differences in the event-related potential (ERP), which describes voltage fluctuations in the ongoing EEG that are time-locked to an event (e.g. the execution of the response or onset of the stimuli), are relatively sparse. There is some evidence that personality traits may be associated with individual changes in the ERP. For instance, the amplitude of P3 (which is a component of the ERP and is related to task-relevant infrequent changes in the stimuli) is weakly associated with individual differences in extraversion (see e.g. Beauducel et al., 2006; Ditraglia \& Polich, 1991; Polich \& Martin, 1992). Extraversion is part of the FFM of 
personality which received growing acceptance from a psychometric point of view in past decades. The FFM incorporates extraversion and neuroticism, which are similar to two of Eysenck's dimensions, together with conscientiousness, openness to experience and agreeableness (Costa \& McCrae, 1992). Taking a closer look at theoretical and empirical approaches, there have been very few studies examining the physiological basis of conscientiousness.

The present work addresses the question whether there is an electrophysiological signature that is related to conscientiousness. For this purpose, I wanted to study components of the ERP that are related to task performance. These components include the error-related negativity (ERN; Falkenstein, Hohnsbein, Hoormann, \& Blanke, 1990, 1991; Gehring, Coles, Meyer, \& Donchin, 1990; Gehring, Goss, Coles, Meyer, \& Donchin, 1993) accompanying errors and the correct-response negativity (CRN; Falkenstein, Hoormann, Christ, \& Hohnsbein, 2000; Ford, 1999; Vidal, Hasbroucq, Grapperon, \& Bonnet, 2000) accompanying correct responses in the performance of choice-reaction tasks. It is of particular interest to observe conscientious individuals in situations where they are forced to do something they would not do under common circumstances, e.g. when they are forced to violate rules they have internalized previously. Rule violations have been shown to evoke a higher response conflict in individuals (Jusyte et al., 2017; Pfister, Wirth, Schwarz, Steinhauser, \& Kunde, 2016; Wirth, Pfister, Foerster, Huestegge, \& Kunde, 2016).

The present thesis first introduces the ERN and CRN and significant ERP components that are related to them. It then continues with two studies trying to observe individual differences in the ERP that might be associated with conscientiousness. The first study tries to examine whether conscientiousness is a correlate of ERN and CRN or both. The second study was aimed to reproduce results from the first study and take a closer look at the individual differences in the ERP associated with conscientiousness. Finally, I try to detect a signature of conscientiousness in the data recorded from both studies applying established methods of machine learning, linear discriminant analysis and logistic regression. 


\subsection{The error-related negativity (ERN)}

This section describes the the ERN/CRN and related ERP-components. Recent theoretical approaches that have been proposed to explain the ERN are reviewed. The section closes with a description of what is already known about variations in the ERN/CRN and factors that cause them.

\subsubsection{The ERN}

The ERN (Gehring et al., 1990; Gehring et al., 1993) which is also referred to as error negativity (Ne; Falkenstein et al., 1990, 1991) is a response-locked negative deflection of the ERP that can be observed after the commission of an error. It occurs within $100 \mathrm{~ms}$ following erroneous responses and its scalp distribution is maximal at midline frontocentral scalp locations. It is assumed to represent the activity of a generic and modality independent response monitoring system as it is observed across stimulus (e.g. Falkenstein et al., 1991; Gehring et al., 1993) and response modalities (e.g. Holroyd, Dien, \& Coles, 1998) and also across different levels of task difficulty (e.g. Falkenstein et al., 2000). The ERN is followed by the error positivity (Pe), a positive deflection that is usually peaking around 150-350ms after response onset at parietal scalp locations (Falkenstein et al., 1991; Overbeek, Nieuwenhuis, \& Ridderinkhof, 2005). It is believed to reflect error awareness (Steinhauser \& Yeung, 2010) or confidence about response correctness (Boldt \& Yeung, 2015).

\subsubsection{Neuroanatomical substrates}

The ERN is suggested to originate in the anterior cingulate cortex (ACC). This assumption is derived from application of different techniques like source localization (Dehaene, Posner, \& Tucker, 1994; Holroyd et al., 1998; Pizzagalli, Peccoralo, Davidson, \& Cohen, 2006; Rüsseler, Kuhlicke, \& Münte, 2003), magnetoencephalography (Miltner et al., 2003), time-frequency analyses (Luu, Tucker, \& Makeig, 2004; Trujillo \& Allen, 2007), and intracerebral recording (Brázdil, Roman, Daniel, \& Rektor, 2005). With its interconnections to limbic and prefrontal areas, the ACC responds to cognitive conflict and affective aversive information (Bush, Luu, \& Posner, 2000). 
Introduction

Activation of the ACC is observed in situations involving response conflict, negative feedback, pain, and error. Therefore, the ERN is thought to reflect a functional interaction of cognitive and affective processes during detection of errors (Hajcak \& Foti, 2008; Hajcak, Moser, Yeung, \& Simons, 2005; Hajcak, Nieuwenhuis, Ridderinkhof, \& Simons, 2005; Luu, Collins, \& Tucker, 2000; Luu, Flaisch, \& Tucker, 2000; Luu, Tucker, Derryberry, Reed, \& Poulsen, 2003). There are two functional subdivisions in the ACC, both of which might generate the ERN. The dorsal and rostral subdivisions have been linked to cognitive and affective processes, respectively (Bush et al., 2000). Studies using fMRI and source localization have indicated that the ERN is generated by the dorsal ACC supporting the notion that the ERN reflects more cognitive processes, while other studies have found the rostral ACC to be the principal generator of the ERN supporting the notion that the ERN is linked to affective processes (Ridderinkhof, Ullsperger, Crone, \& Nieuwenhuis, 2004; Taylor, Stern, \& Gehring, 2007).

There is evidence that lateral prefrontal cortex also critically contributes to error monitoring processes (Carter et al., 1998; Gehring \& Knight, 2000; Turken \& Swick, 2008; Ullsperger \& Cramon, 2006). The ability to process errors is associated with balancing of internal representations of task instructions and goals which is depending on structures such as the PFC (Weinberg, Riesel, \& Hajcak, 2012).

There are dopaminergic neuron innervations in the ACC (Allman, Hakeem, Erwin, Nimchinsky, \& Hof, 2001). The views that dopamine is related to reward are consistent with theoretical accounts suggesting that the ERN displays dopaminergic disinhibition of ACC neurons when the outcome of actions is evaluated as worse than predicted (Holroyd $\&$ Coles, 2002).

\subsubsection{Correct-response negativity}

Correct responses often trigger a negativity that has an equal latency in the responselocked waveform as the ERN but with a smaller amplitude (Falkenstein et al., 2000; Ford, 1999; Vidal et al., 2000). Both components have a similar topography (Luu, Flaisch et al., 2000; Vidal et al., 2000) and presumably the same source in the ACC (Roger, Bénar, Vi- 
dal, Hasbroucq, \& Burle, 2010). Their waveforms are strikingly similar, particularly in individual subject data (Swick \& Turken, 2002). The component is called correct-response negativity (CRN; Ford, 1999). Of note, ERN is much larger on error trials than CRN on correct trials.

It has also been shown that partial errors (i.e. when subjects initiated an incorrect response but self-corrected before errors were committed or correct responses that are perceived as errors by the participant) can generate a component that is equal to the ERN in terms of its amplitude (Burle, Roger, Allain, Vidal, \& Hasbroucq, 2008; Carbonnell \& Falkenstein, 2006; Gehring et al., 1993; Scheffers, Coles, Bernstein, Gehring, \& Donchin, 1996). On a flanker task where e.g. stimuli consist of arrows, incongruent stimuli (i.e. target arrow points to a different direction than the surrounding arrows, e.g. " $<<><<$ "; task: respond to the central stimulus: > right key, $<$ left key) elicit a larger CRN than congruent stimuli (i.e. central and surrounding arrows pointing to the same direction, e.g. " $<<<<<$ "; Bartholow et al., 2005). CRN is largest when the presented trial type was different than expected (i.e. when an incongruent trial appeared while a congruent trial was expected). This observation showed that changes in CRN amplitude from trial to trial not only reflect response conflict but also strategic conflict. According to control models (Kerns et al., 2004; Ridderinkhof et al., 2004) either type of conflict is sufficient to elicit a signal from the ACC to the prefrontal cortex (PFC) indicating that an increase in attention is required.

Trials directly preceding errors had a characteristic positivity just following the CRN. This error-preceding positivity (EPP) can be interpreted as disengagement of the ACC from the response monitoring process (Ridderinkhof, Nieuwenhuis, \& Bashore, 2003).

It has been observed that an inverse relationship between the probability of committing an error and the magnitude of the CRN exists (Allain, Carbonnell, Falkenstein, Burle, \& Vidal, 2004). Larger CRN amplitudes may have a preventive function against errors and the magnitude of CRN may indicate the extent of engagement of monitoring on correct trials (Simons, 2010).

Due to their similarities some authors assume that ERN and CRN are not distinct components but reflect an identical cognitive control process during response monitoring that is 
specifically enhanced on error trials (Burle et al., 2008; Meckler et al., 2011; Suchan, Jokisch, Skotara, \& Daum, 2007). However, there is also evidence that ERN and CRN are distinct, i.e. reflecting different processes (Vocat, Pourtois, \& Vuilleumier, 2008; Yordanova, Falkenstein, Hohnsbein, \& Kolev, 2004). Another perspective is that both components reflect the combined activity of two underlying processes each reflecting different aspects of performance monitoring (Endrass, Klawohn, Gruetzmann, Ischebeck, \& Kathmann, 2012).

\subsubsection{Theoretical approaches of the ERN}

Several theories explaining the functional significance of the ERN have been proposed. Three of them are described in this chapter: Error detection theory, conflict monitoring theory and reinforcement learning theory.

\subsubsection{Error detection theory}

As one of the earliest approaches, error detection theory assumes a comparator system that constantly evaluates the mental representations of the correct and the actual response. According to this theory the ERN is elicited in case of a mismatch between both representations (e.g. Bernstein, Scheffers, \& Coles, 1995; Coles, Scheffers, \& Holroyd, 2001; Falkenstein et al., 1991) and corrective or compensating actions are initiated. The comparison process is illustrated in Figure 1. Errors are caused by impulsive responses due to incomplete stimulus processing. The approach was challenged by observations of the CRN in absence of an error or an obvious response conflict (Vidal, Burle, Bonnet, Grapperon, \& Hasbroucq, 2003) leading to an adaptation of the error detection theory in which ERN and CRN reflect the comparison process itself rather than its outcome. In this process, the ERN indicates the comparison process in combination with an additional error signal which may be unique to error trials (Falkenstein et al., 2000). 


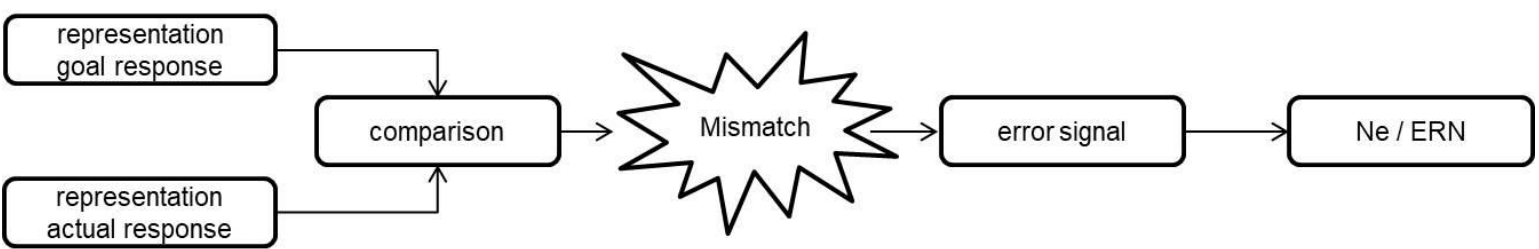

Figure 1. Illustration of the error detection theory of the ERN proposed by Coles et al. (2001) assuming a comparison process that evaluates the consistency of the representation of the actual response and the representation of the correct response. Inconsistency of both representations elicits a mismatch or error signal which is reflected by the ERN. Adapted from de Bruijn, Miedl, and Bekkering (2011).

\subsubsection{Conflict monitoring theory}

An alternative explanation of the occurrence of the ERN is provided by conflict monitoring theory. It accounts for the computational implausible fact that if there was a comparator successfully checking whether the response was correct or not, it had to have the information about the correct response representation. Thus, the comparator would have access to information outside of the response monitoring system. The question then arises why the correct response was not executed, if this system had information about the intended (correct) response. The conflict monitoring approach assumes a response conflict (a concurrent activation of multiple competing responses) that is triggered by typical choice tasks and signals the need for increased control (Botvinick, Braver, Barch, Carter, \& Cohen, 2001; Carter et al., 1998; Yeung, Botvinick, \& Cohen, 2004) and gives rise to the ERN. Response conflict can also be observed on a behavioral level when participants often correct their errors, although they were not instructed to do so (Fiehler, Ullsperger, \& Cramon, 2005).

The time course of the ERN amplitude and its sensitivity to several experimental manipulations were simulated by models based on the conflict monitoring theory (Yeung et al., 2004). Conflict models were also used to simulate performance in a wide variety of tasks (Botvinick et al., 2001). These computational models of the conflict monitoring approach (see Figure 2) use parallel distributed processing networks to model response selection. According to these models, response conflict is defined as the Hopfield energy of the response units. In a simple two-choice task, this energy is calculated as twice the product 
of the activation of two response units, weighted by the strength of the inhibitory connections among responses (e.g. Botvinick et al., 2001; Yeung et al., 2004).

The conflict-monitoring approach hypothesizes that the conflict is monitored by the ACC (Carter \& van Veen, 2007; Yeung et al., 2004). When the ACC detects increased conflict, projections to prefrontal cortex signals the need for adjustments in cognitive control (Botvinick et al., 2001; Carter et al., 1998; Yeung et al., 2004). There is evidence that dorsolateral prefrontal cortex activity on subsequent trials and post-error slowing is associated with ACC activity following errors (Kerns et al., 2004; van Veen \& Carter, 2006). In addition, data from neuroimaging studies indicated ACC activity on both error trials and correct trials with incongruent stimuli eliciting high levels of response conflict (Botvinick, Nystrom, Fissell, Carter, \& Cohen, 1999; Carter et al., 1998; MacDonald, Cohen, Stenger, \& Carter, 2000).

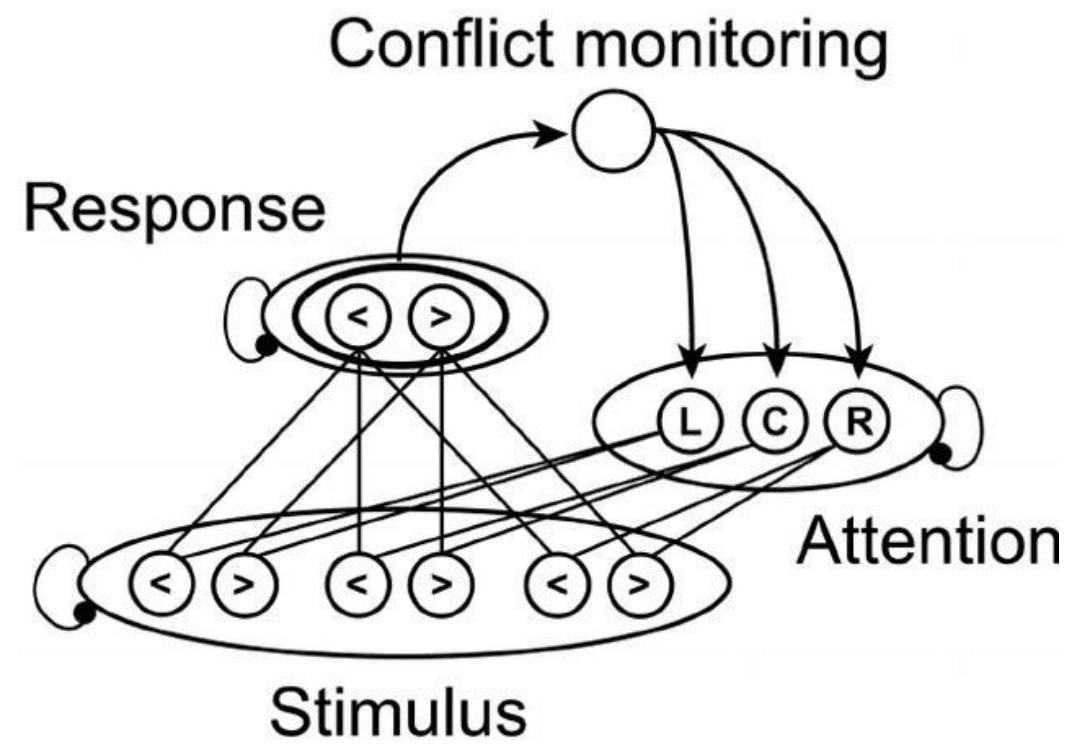

Figure 2. Illustration of a conflict-monitoring model for the Eriksen Flanker task. Responses are activated by corresponding stimulus processing units that correspond to the target and flanker triangles $<$ and $>$. Coactivation of the mutually incompatible response units $(<$ and $>$ ) generates the conflict. The presence of conflict is detected by the conflict-monitoring process which then signals adjustments in attention to reduce conflict in the subsequent trials. Adapted from Yeung and Nieuwenhuis (2009). 
However, there are some studies that question the conflict monitoring approach. Carbonnell and Falkenstein (2006), for instance, measured no difference in the size of the ERN magnitude when comparing a correct response with a previous more forceful error in one case and a previous less forceful error in the other. Similarly, Masaki and colleagues (2007) found no difference in the ERN amplitude between two trial types, although they measured a greater conflict in the more difficult one using electromyography. A study of Burle and coworkers (2008) was based on simulations establishing the amount of response conflict during two conditions where the ERN amplitudes are compared. According to their simulations there is less response conflict when the interval between an error and the error correction is long than when both responses occur close together. However, the authors observed a contrasting pattern consisting of a smaller ERN amplitude when the interval between error and correction was short than when it was long.

\subsubsection{Reinforcement learning theory}

According to reinforcement learning theory (RL-ERN) an error signal is produced by monitoring mechanisms and is triggered when events occur that violate the expectations. These expectations associated with a response are formed by prior reinforcements. In an early learning phase errors may occur because the system has not yet learnt the stimulusresponse-consequence (S-R-C) contingencies. In this stage, the system needs external feedback to evaluate its performance (i.e. determine whether it is good or bad). After S-R$\mathrm{C}$ associations are strengthened, errors can be detected as soon as a response occurs and without help by external feedback. The location of this monitoring mechanism assumingly is in the basal ganglia (see Figure 3). An error signal is conveyed to the ACC by the midbrain dopamine system. In the ACC the signal is used to increase task performance by influencing how control over the motor system is allocated to different competing systems in the brain (Holroyd \& Coles, 2002; Holroyd, Yeung, Coles, \& Cohen, 2005). 


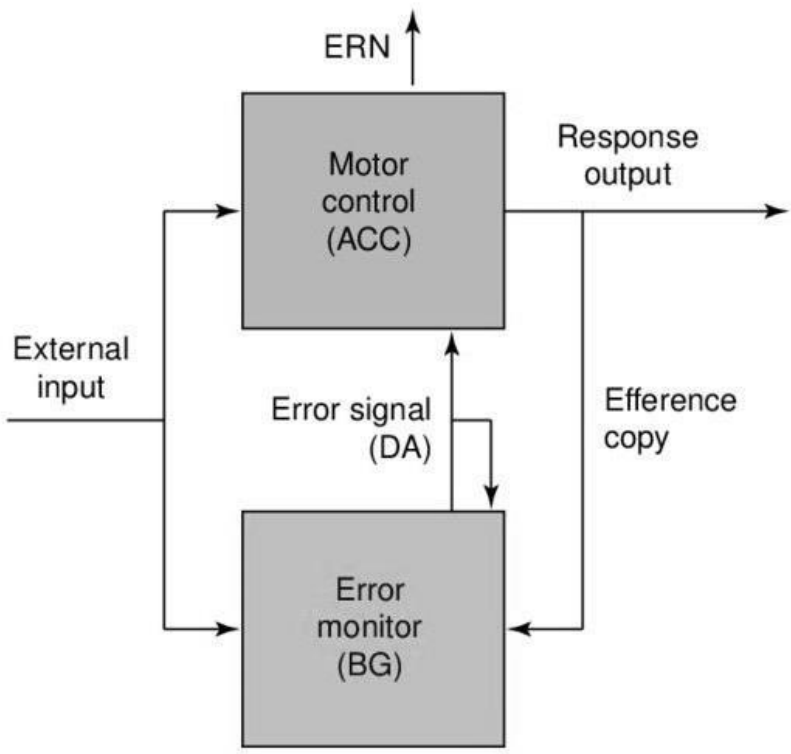

Figure 3. Illustration of the reinforcement-learning theory proposed by Holroyd and Coles (2002). A mechanism in the basal ganglia (BG) monitors the performance of a mechanism located in the anterior cingulate cortex (ACC) that maps external input into response output. An error signal is conveyed to the ACC by the midbrain dopamine system. The theory assumes that the ERN is produced by the ACC. Adapted from Holroyd and Yeung (2003).

\subsubsection{Association of ERN and behavior}

According to RL-ERN as well as conflict monitoring accounts, variation in the amplitude of the ERN is associated with current behavior and the ERN itself serves to form subsequent behavior. Adaptive responses to errors, like post-error slowing, are behavioral adjustments to improve task performance (Holroyd et al., 2005; Holroyd \& Coles, 2002). Post-error slowing is assumed to reflect an increase in response caution in order to increase the probability of correct response in a subsequent trial at the expense of response speed. However, the relationship between variation in the ERN and behavioral measures still remains unclear because multiple instances exist in which variation in the ERN occurs although behavioral differences are absent (for a review see Weinberg et al., 2012). While the mechanisms responsible for the generation of the ERN appear to be similar across individuals, there is evidence that the ERN amplitude is moderated by situational, motivational and affective processes, as well as more stable traits that differ inter-individually. There is substantial evidence that the functional significance of variation in the ERN is related to dispositional characteristics (Weinberg et al., 2012). Neither RL-ERN nor conflict moni- 
toring theories adequately explain the individual differences that have been observed in the ERN.

\subsubsection{ERN / CRN and individual differences}

The ERN amplitude seems to be affected by motivational salience. It is enhanced when error significance is emphasized, either through task instructions that stress accuracy over speed (Falkenstein et al., 2000; Gehring et al., 1993), external performance evaluation (Hajcak, Moser et al., 2005; Kim, Iwaki, Uno, \& Fujita, 2005), or incentives (Chiu \& Deldin, 2007; Endrass et al., 2010; Ganushchak \& Schiller, 2008; Hajcak, Moser et al., 2005; Pailing \& Segalowitz, 2004; Riesel, Weinberg, Endrass, Kathmann, \& Hajcak, 2012).

Amplitude of the ERN also appears to be increased in individuals who experience errors as more aversive than other individuals. A recent meta-analysis suggests that a larger ERN is related to anxiety, specifically apprehension/worry, while a larger CRN is not reliably associated with anxiety (Moser, Moran, Schroder, Donnellan, \& Yeung, 2013). Enhanced ERN amplitude has been also observed in individuals with obsessive-compulsive disorder (OCD; e. g. Endrass et al., 2010; Endrass, Klawohn, Schuster, \& Kathmann, 2008; Gehring, Himle, \& Nisenson, 2000; Johannes et al., 2001; Riesel, Endrass, Kaufmann, \& Kathmann, 2011; Ruchsow et al., 2005). ERN is also positively related to symptom severity in OCD patients (Gehring et al., 2000). Healthy individuals scoring high in emotionality and negative affect (e.g. anxiety and fear; Luu, Collins et al., 2000) or with increased scores on the Behavioral Inhibition System scale are also characterized by enhanced ERN amplitudes (Boksem, Tops, Wester, Meijman, \& Lorist, 2006). During tasks that penalize error responses, reduced ERN amplitudes are associated with low scores on trait socialization (Dikman \& Allen, 2000) indicating a smaller sensitivity to punishment.

ERN amplitude has also been shown to vary with personality dispositions. Motivational manipulations that impact ERN amplitude may be moderated by personality traits (Pailing \& Segalowitz, 2004). As an example, Olvet and Hajcak (2011) observed that the impact of sad mood on ERN amplitude is moderated by neuroticism. 
In a recent review, Weinberg et al. (2012) assumed that a reduced ERN, related to externalizing traits and psychopathology, may reflect motivational disengagement, disinhibition, and decreased conscientiousness. Furthermore, enhanced ERN may reflect characteristics that are common to anxiety disorders such as perfectionism, concern over errors, negative affect and increased intolerance of uncertainty. These characteristics may have a strengthening effect on error significance. Poorer performance and smaller ERN may therefore both be caused by task disengagement and motivational deficits, whereas larger ERNs maybe linked to increased motivational significance of errors.

To date, the relationship of individual differences and CRN amplitude has not been explored in detail. There is evidence that ERN and CRN amplitudes are larger in participants with obsessive-compulsive characteristics (Hajcak \& Simons, 2002) and bilinguals (Kałamała, Drożdżowicz, Szewczyk, Marzecová, \& Wodniecka, 2018) indicating differences in task strategy and higher task engagement.

Additionally, while there is a huge body of research concerned with the relationship of psychopathology and other individual differences with ERN, there are only a few studies examining the role of personality traits, especially conscientiousness, in the generation of the ERN amplitude. Hill, Samuel, and Foti (2016) found that at low levels of conscientiousness, negative urgency (i.e. impulsivity in connection with negative affect) had a positive impact on the magnitude of the ERN. Pailing and Segalowitz (2004) observed that individuals higher on conscientiousness were characterized by smaller motivation-related changes in the ERN across monetary incentives. 


\section{Performance monitoring and correct response significance in conscientious individuals}

\subsection{Introduction}

In Experiment 1, I wanted to examine whether conscientiousness is related to an increased motivational salience of an error and whether individuals scoring high on a conscientiousness scale display a stronger task engagement than individuals with low levels of conscientiousness. Increased motivational salience is associated with enhanced ERN and accompanied with increased error significance and stronger task engagement (Weinberg et al., 2012). Individuals scoring high on conscientiousness may thus have a larger ERN or even a larger CRN amplitude.

Furthermore, I wanted to investigate the relationship of rule violations and medialfrontal response-locked ERPs. The ERN appears to elicit after slips during unwilled actions but not after mistakes during willed actions (Stemmer, Witzke, \& Schönle, 2001). However, violating a rule may evoke a higher response conflict in individuals (Jusyte et al., 2017; Pfister, Wirth, Schwarz, Steinhauser et al., 2016; Wirth et al., 2016) and stronger response conflict is associated with a larger magnitude of ERN and CRN (Bartholow et al., 2005). According to conflict monitoring accounts, violating a rule could be reflected by variations in the magnitude of ERN and CRN. I wanted to determine whether there is an enhanced CRN when violating a rule compared to rule compliant behavior. Especially participants with high levels of conscientiousness may have a higher response conflict when they are forced to violate a rule and may thus have an even more enhanced CRN during rule violations. 
$\underline{\text { Performance monitoring and correct response significance in conscientious individuals }}$

In the present study, a choice reaction task establishing a rule with clear S-R contingency was designed. Participants had to respond to a circle by pressing the left key, to a square by pressing the right key. In two other conditions participants had to either violate or reverse this rule. It is particularly significant that the S-R mapping was the same in both alternative conditions. The only way to follow the instructions and violate the rule was doing the opposite as in the standard condition, i.e. pressing the left key when a square occurred, pressing the right key when a circle appeared. The alternative conditions allowed me to compare rule-consistent with rule-violation behavior. Importantly, both alternative conditions required the same response. If there was a behavioral or electrophysiological effect of rule violations it should have an impact on the difference in response times and error rates and the response-locked medial-frontal ERP. To examine the relationship of conscientiousness with response monitoring, the conscientiousness scale of the NEO Five Factor Inventory was administered (NEO-FFI; Borkenau \& Ostendorf, 2008) to the sample.

I expected generally prolonged RTs and higher error rates for rule violations compared to rule-compliant behavior. The medial-frontal response-locked ERP during rule violations in both erroneous and correct responses presumably show an accentuated negativity in a time window of $0-100 \mathrm{~ms}$ (corresponding to ERN and CRN) compared to the rule-based conditions. Additionally, I expected participants scoring high on the conscientiousness scale being faster in responses and committing fewer errors reflecting higher task engagement and greater concern over errors. At the same time, participants with high conscientiousness values were assumed to show prolonged post-error slowing. I also assumed that individual differences in the medial-frontal response-locked ERP after erroneous and correct responses were associated with conscientiousness. More specifically, ERN and CRN measured at frontocentral electrodes were hypothesized to be more pronounced in individuals with high levels of conscientiousness. 


\subsection{Materials and Methods}

\subsubsection{Participants}

46 participants (42 females, 2 left-handed) between the ages of 18 and 47 years $(M=22.6, S D=8.5)$, mainly undergraduate university students were recruited and received 2.5-hr course credit. All participants reported that they were free of neurological disorders and had normal or corrected-to-normal visual ability. One participant had to be excluded from EEG analysis due to current use of psychoactive medication. I had to exclude another 18 participants (39\%) from all analyses due to commission of too few errors $(<5)$ in either of both alternative instruction conditions RV and RR (see below). This massive shrinkage of the sample may have been due to a rather low level of task difficulty while at the same time the absolute number of trials in some conditions was low (120). All participants were naive concerning the hypotheses underlying the experiment and had signed a consent form prior to participation in the study. The participants were informed that they could withdraw from the study at any time without penalty or loss of privilege.

\subsubsection{Materials}

\subsubsection{Questionnaires}

The NEO-FFI (Borkenau \& Ostendorf, 2008, original version by Costa \& McCrae, 1992) was used. It is a shorter version of the Revised NEO Personality Inventory (NEO-PI$\mathrm{R}$; Costa \& McCrae, 1992) with 60 items derived from the original 240 items. The five domains assessed with 12 items each by the NEO-FFI are conscientiousness, extraversion, agreeableness, neuroticism, and openness. Each item is rated on a 5-point scale.

\subsubsection{Stimuli and Apparatus}

Stimuli were displayed on a 24" screen with a resolution of 1600 x 900 pixels and participants had an average viewing distance of $65 \mathrm{~cm}$ to screen. A button box (Cedrus RB830, San Pedro, CA) served as response device. Stimuli consisted of a green circle with a diameter of $28 \mathrm{~mm}$ or a green square that measured $28 \times 28 \mathrm{~mm}$. This corresponds to a display size of $2.5^{\circ}$ of visual angle for the stimuli and $9.0^{\circ} \times 3.0^{\circ}$ of visual angle for in- 
$\underline{\text { Performance monitoring and correct response significance in conscientious individuals }}$

structions. There was one button each for left and right responses. Participants were asked to respond bimanually and to use the index finger of each hand to press the buttons. The randomized presentation of stimuli and instructions was controlled by Presentation Version 16 (Neurobehavioral Systems, Inc., Albany, CA). The correct response was determined by the target stimulus and one of three instructions. Participants were instructed to respond in accordance with the overall rule that required to press the left button when the stimulus was a circle and to press the right button when the stimulus was a square.

\subsubsection{Procedure}

The trial procedure is illustrated in Figure 4. Trials started with a blank screen (500ms), followed by the presentation of an instruction $(1500 \mathrm{~ms})$ according to one of the three conditions (see below). After that, a fixation cross was presented $(500 \mathrm{~ms})$ and the target stimulus (circle or square) followed. The target remained on screen until a response was given. Possible response types were pressing the left or the right button to the target that was either a circle or a square. 


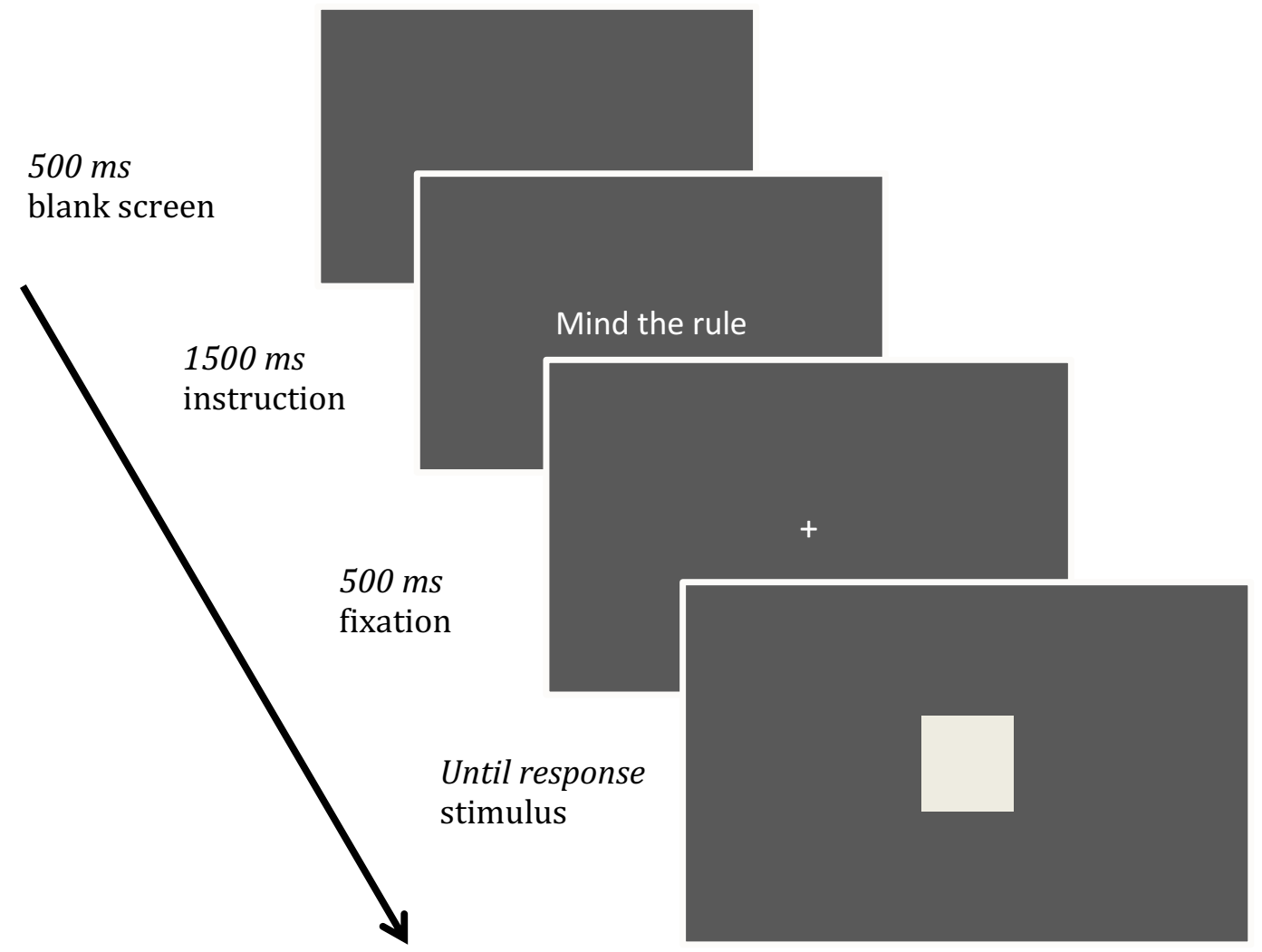

Figure 4. Procedure of a trial. After the presentation of a blank screen $(500 \mathrm{~ms})$, the participant is instructed by a short text $(1500 \mathrm{~ms})$. Then the presentation of a fixation cross $(500 \mathrm{~ms})$ follows whereafter the target stimulus is presented until the participant responds.

The experimental conditions were administered as follows. Depending on the condition, instruction and correct answers were:

- Standard (STD): "Mind the rules" (German "Beachte die Regeln"), circle - left, square - right

- Rule violation (RV): “Commit an error" (German "Mache einen Fehler”), circle right, square - left

- Rule reversal (RR): "Reverse the rules" (German "Vertausche die Regeln"), circle - right, square - left

The experiment consisted of 30 blocks of 20 trials each (600 trials in total). The standard condition included $60 \%$ of the trials whereas the other two tasks comprised $20 \%$ each. All trials were presented in a randomized order across the experiment. The visual stimuli (circles and squares, 300 each) were equally distributed over all conditions. The experi- 
$\underline{\text { Performance monitoring and correct response significance in conscientious individuals }}$

mental manipulation was implemented in terms of different instructions across trials. Before the experiment started, participants had to practice the mapping rules in a training phase of 20 trials. During the experiment, participants determined the length of breaks between blocks themselves.

\subsubsection{EEG recording and data analysis}

EEG was recorded by means of an EasyCap (EASYCAP GmbH, Germany) equipped with sintered Ag-AgCl-electrodes. For the recording I used electrodes F1, F2 C3, C4, CP1, CP2, CP5, CP6, Cz, F3, F4, F7, F8, FC5, FC6, FCz, Fz, O1, O2, P3, P4, P7, P8, Pz, T7, T8, TP10, TP9 placed according to the 10-20-system of electrode placement (Jasper, 1958). In addition, two electrodes were placed above the left and right mastoid. The electrode $\mathrm{AFz}$ was used as ground and an electrode on the nose tip as reference. However, only recorded data from electrodes $\mathrm{FCz}$ and $\mathrm{Pz}$ were used for statistical analysis as the components of interest showed a clear amplitude maximum at these positions. The EEG signal was amplified by a BrainAmp amplifier (Brain Products $\mathrm{GmbH}$, Germany) with a sampling rate of $250 \mathrm{~Hz}$ and $16 \mathrm{bit} / \mathrm{channel}$. Using a band-pass filter of $0.01 \mathrm{~Hz}$ to $30 \mathrm{~Hz}$ the signal was filtered to eliminate skin conductance and muscle activity interference. Additionally, a notch filter with $50 \mathrm{~Hz}$ was used. All impedances were maintained below $10 \mathrm{k} \Omega$ during the entire recording procedure. After the experiment, EEG data were further prepared in BrainVision Analyzer 2.0.1 (Brain Products GmbH, Germany). Only trials with responses between $200 \mathrm{~ms}$ and $1500 \mathrm{~ms}$ after stimulus presentation were considered for analysis. Before ERP data analysis, all trials containing eye-movement artifacts ("blinks") were corrected using a blind component separation (Joyce, Gorodnitsky, \& Kutas, 2004), which has been shown to be superior to other artifact correction procedures (Kierkels, van Boxtel, \& Vogten, 2006).

For purpose of data analysis we averaged waveforms with a $100 \mathrm{~ms}$ pre-response baseline to $600 \mathrm{~ms}$ post-response. A baseline correction was applied to the $100 \mathrm{~ms}$ pre-response interval. Epochs with voltage steps of $20 \mu \mathrm{V} / 4 \mathrm{~ms}$ or differences of $300 \mu \mathrm{V}$ after independent component analysis (ICA) correction in an interval of $150 \mathrm{~ms}$ on each channel were rejected from further data analysis. 
$\underline{\text { Performance monitoring and correct response significance in conscientious individuals }}$

I analyzed the error-related components ERN and Pe and the component CRN which is the counterpart of ERN elicited by correct responses. The ERN/CRN is observed as the maximum amplitude of the negative deflection within the first $100 \mathrm{~ms}$ after (incorrect) response onset over fronto-central electrodes along the midline (electrodes $\mathrm{Fz}$ and $\mathrm{FCz}$ ). The ERN/CRN was measured in response-locked ERP averages. The Pe follows the ERN and is a positive deflection, usually peaking around $150-350 \mathrm{~ms}$ after response onset. Its maximum amplitude is observed over centro-parietal electrodes along the midline (electrodes $\mathrm{Cz}$ and $\mathrm{Pz}$ ). Depending on the electrophysiological properties of the present ERP dataset, I defined the ERN/CRN as the mean amplitude during 20-70ms post-response interval at electrode FCz. The Pe/Pc was calculated as the mean amplitude during 200-350ms interval following response onset at electrode $\mathrm{Cz}$. Table 1 show mean trial numbers that were included in the analysis.

Table 1. Mean number of trials for each combination of instruction condition and response type that were included in statistical analyses.

\begin{tabular}{lcccccccc}
\hline & \multicolumn{3}{c}{ Correct } & & \multicolumn{3}{c}{ Error } \\
\cline { 2 - 4 } \cline { 7 - 9 } Condition & Mean & Min & Max & & Mean & Min & Max \\
\hline Standard & 337.18 & 298 & 356 & & 10.62 & 5 & 39 \\
Rule violation & 107.89 & 95 & 118 & & 7.19 & 5 & 29 \\
Rule reversal & 106.86 & 88 & 116 & & 8.00 & 5 & 32 \\
\hline
\end{tabular}

For all response time (RT) analyses, data was corrected for outliers by removing trials with RTs that deviated more than 2.5 SDs from the mean RT of each participant and condition.

Typically, RTs after erroneous responses are slower compared to RTs after correct responses (post-error slowing). I defined the measure of post-error slowing (PES) as follows:

$$
\text { PES }=\mathrm{RT}_{\text {Correct }(\mathrm{STD}) \rightarrow \operatorname{Correct}(\mathrm{STD})}-\mathrm{RT}_{\text {Error(STD) } \rightarrow \operatorname{Correct}(\mathrm{STD})}
$$

I assessed effects of rule condition (STD vs. RV vs. RR), conscientiousness (continuous) and accuracy (error vs. correct) as well as their interactions on response-locked ERPs via linear mixed-effects regression using the lme4 package in $\mathrm{R}$ (Version 3.5.1; Bates, 
$\underline{\text { Performance monitoring and correct response significance in conscientious individuals }}$

Mächler, Bolker, \& Walker, 2014). In addition, I analyzed effects of rule condition (STD vs. RV vs. RR) and conscientiousness (continuous) including their interactions on the behavioral measures error rate and RT also by means of linear mixed-effects regression. Linear mixed-effects regression provides several important advantages over traditional methods such as repeated-measures ANOVA; it allows including conscientiousness as a continuous variable and rule condition as repeated-measures variable. I obtained $p$ values for all linear mixed-effects models using the anova function of the lmerTest package (Kuznetsova, Brockhoff, \& Christensen, 2017) with a Kenward-Roger approximation of degrees of freedom. As random effects, I defined intercepts for subjects, as well as by-subject random slopes for the effect of conscientiousness and rule condition. Furthermore, mean values of PES were analyzed via linear regression with regressing PES onto conscientiousness.

\subsection{Results}

\subsubsection{NEO-FFI scales}

Sufficient ranges in conscientiousness (Min $=22, \operatorname{Max}=48, M D=36, M=35.47, S D$ $=6.90$ ) obtained from the sample with possible ranges of 0 to 48 were achieved. Figure 5 shows the distribution of conscientiousness scores. Descriptive statistics and internal consistency values are provided in Table 2 , intercorrelations among the personality scales in Table 3. A negative relationship between openness and conscientiousness, $r=-.32$ ( $p<$ $.05)$ is the only significant relationship among the measured personality scales. 
$\underline{\text { Performance monitoring and correct response significance in conscientious individuals }}$

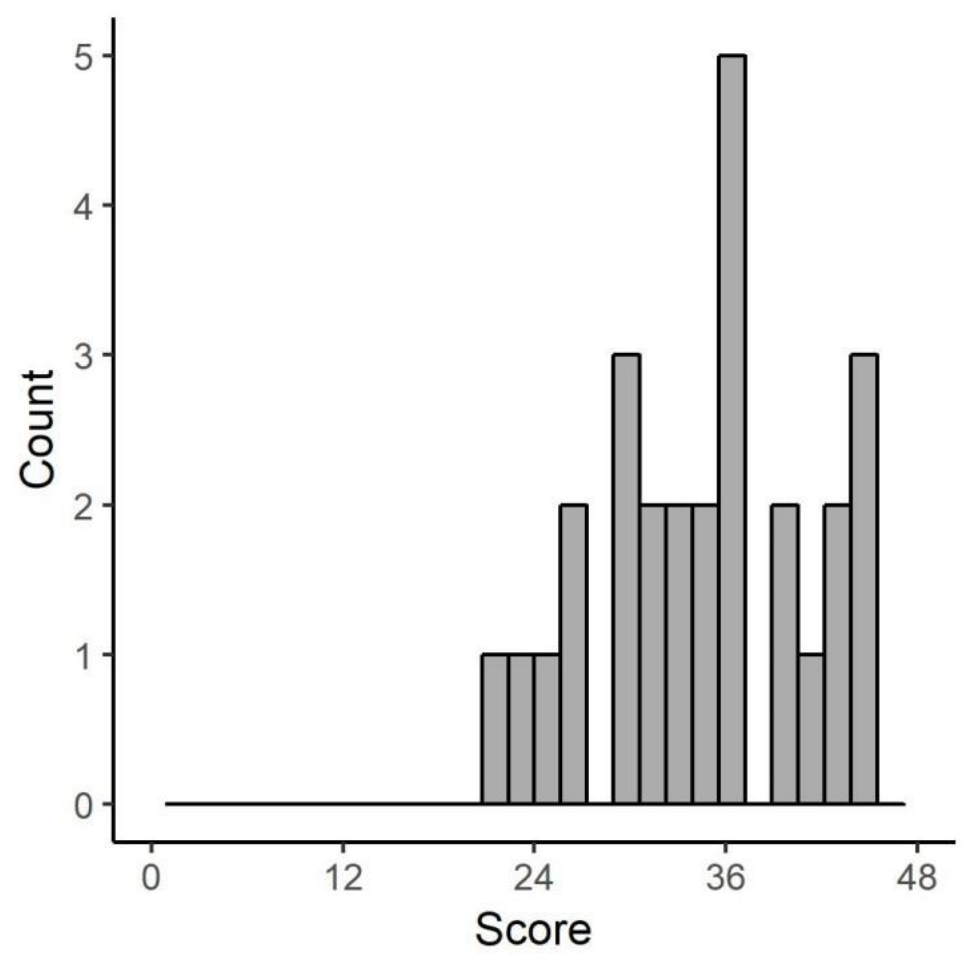

Figure 5. Distribution of conscientiousness scores obtained from the sample.

Table 2. Descriptive statistics (Mean, SD) and internal consistency (cronbach's alpha) for personality traits measured by NEO-FFI.

\begin{tabular}{lcc}
\hline Personality scale & M (SD) & $\alpha$ \\
\hline Neuroticism & $22.49(7.57)$ & .88 \\
Extraversion & $28.82(5.45)$ & .73 \\
Openess & $32.98(6.42)$ & .81 \\
Agreeableness & $34.16(6.43)$ & .84 \\
Conscientiousness & $35.47(6.90)$ & .86 \\
\hline
\end{tabular}


$\underline{\text { Performance monitoring and correct response significance in conscientious individuals }}$

Table 3. Intercorrelations among personality traits measured by NEO-FFI.

\begin{tabular}{lcccc}
\hline Personality scale & EV & ON & AA & CS \\
\hline Neuroticism (NT) & -.20 & .09 & -.24 & .01 \\
Extraversion (EV) & - & .05 & 0.23 & 0.07 \\
Openness (ON) & & - & -0.01 & $-0.32 *$ \\
Agreeableness (AA) & & - & -0.12 \\
Conscientiousness (CS) & & & & - \\
\hline Notes. $n=28 .{ }^{*} p .05$ & & &
\end{tabular}

\subsubsection{Behavioral data}

To analyze the influence of rule condition and conscientiousness on behavioral performance, I performed two linear mixed-effects regression analyses for both error rate and RT (see Figure 6A and B). As fixed effects for each analysis, I entered rule condition and conscientiousness as well as their interaction term. The main effect of conscientiousness approached to be a significant predictor of error rate, $F(1,26)=3.13, p=.09$ whereas other effects remained non-significant, $F \mathrm{~s}<1.40, p \mathrm{~s}>.26$. The analysis of RTs showed that the main effect of rule condition was significant, $F(1,52)=5.97, p=.005$ and other effects remained non-significant, $F \mathrm{~s}<1.69, p \mathrm{~s}>.20$. Bonferroni post hoc tests used to break down the main effect of rule condition revealed that instruction from STD condition which is the frequent one evoked faster responses than instructions from $R R(p<.001)$ and $R V$ ( $p$ $<.001)$. 


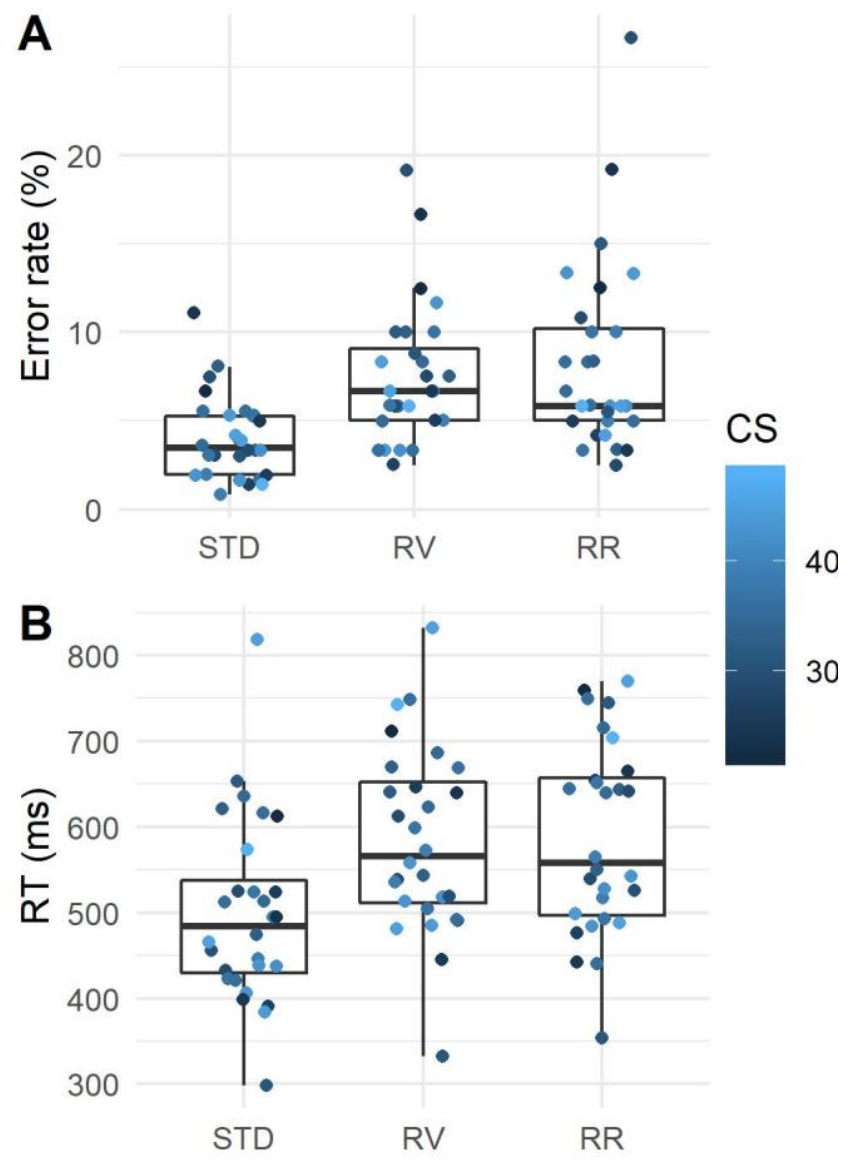

Figure 6. Mean error rates (A) and response times (B) for each instruction condition, standard (STD), rule violation (RV), and rule reversal (RR). Scatter plots display the performance as a function of conscientiousness (CS). See text for details.

As a last component analyzing the predictive ability of conscientiousness on behavioral performance, I ran a simple linear regression analysis with regressing PES onto the conscientiousness values. The main effect of conscientiousness was non-significant, $\beta=-.22$, $t(26)=1.12, p=.27$.

\subsubsection{ERPs}

\subsubsection{ERN / CRN}

I constructed a linear mixed-effects regression model to examine the influence of rule condition, conscientiousness and accuracy on the ERN/CRN amplitude. As fixed effects, I entered rule condition, conscientiousness and accuracy as well as all possible interaction terms. I found a significant main effect of accuracy on the response-locked ERP within 20- 
$\underline{\text { Performance monitoring and correct response significance in conscientious individuals }}$

$70 \mathrm{~ms}$ post-response time interval, $F(1,78)=11.25, p=.001$, with higher amplitude on error trials than on correct trials. I also found a significant interaction of conscientiousness and accuracy, $F(1,78)=4.46, p=.038$. All other effects remained non-significant, $F_{\mathrm{S}}<1.02, p \mathrm{~s}>.36$.

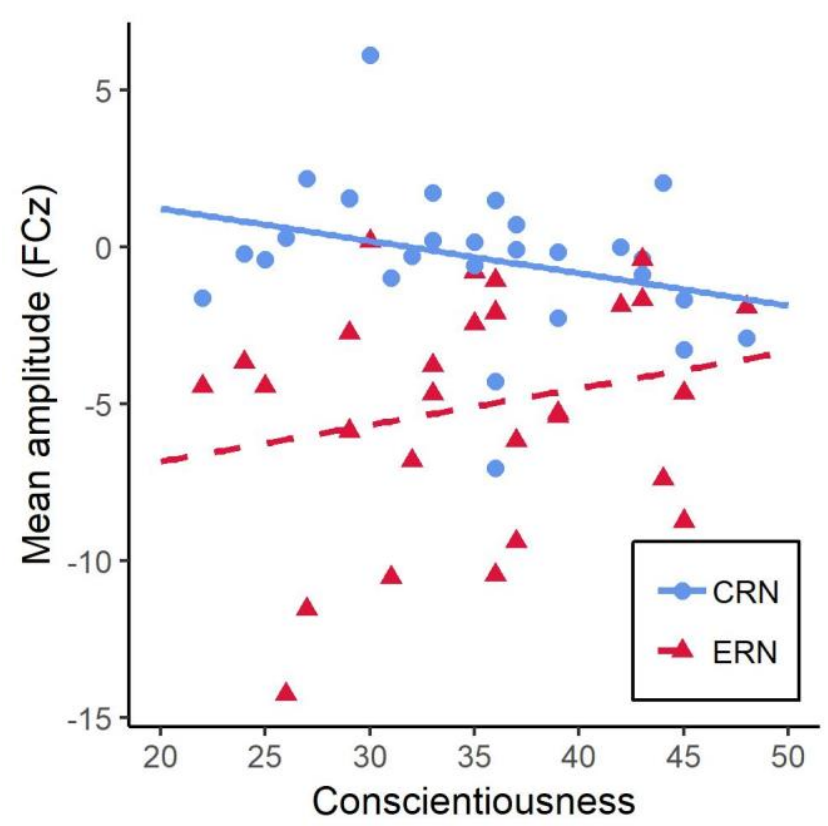

Figure 7. Mean amplitudes during 20-70ms post-response interval at electrode $\mathrm{FCz}$ as a function of conscientiousness for correct (CRN) and incorrect responses (ERN). The lines resulted from regressing CRN and ERN amplitudes onto conscientiousness.

To elucidate the significant interaction of conscientiousness and accuracy, I ran two separate regression analyses to examine the influence of conscientiousness on both the ERN and CRN amplitude. Both regression analyses did not find conscientiousness to be a significant predictor of neither ERN, $\beta=.22, t(26)=1.16, p=.26$, nor CRN amplitude, $\beta=-.30, t(26)=1.62, p=.12$. However, the regression lines depicted in Figure 7 indicated that higher values of conscientiousness were associated with higher CRN and with lower ERN amplitudes while lower values of conscientiousness were associated with lower CRN and higher ERN amplitudes. Additionally, a regression analyses examining the influence of conscientiousness on $\triangle E R N$, the difference score of ERN and CRN amplitudes, found conscientiousness to be a significant predictor, $\beta=.38, t(26)=2.10, p=.046$. 
$\underline{\text { Performance monitoring and correct response significance in conscientious individuals }}$

\subsubsection{Pe / Pc}

To investigate whether there is an influence of conscientiousness during the rule conditions on the $\mathrm{Pe} / \mathrm{Pc}$ amplitude, I built a linear mixed-effects regression model with fixed effects rule condition, conscientiousness and accuracy as well as all possible interaction terms. It revealed a significant interaction effect of conscientiousness and accuracy, $F(1,78)=5.28, p=.024$. The main effect conscientiousness approached significance, $F(1,26)=3.01, p=.09$. All other effects remained non-significant, $F_{\mathrm{S}}<1.68, p \mathrm{~s}>.19$.

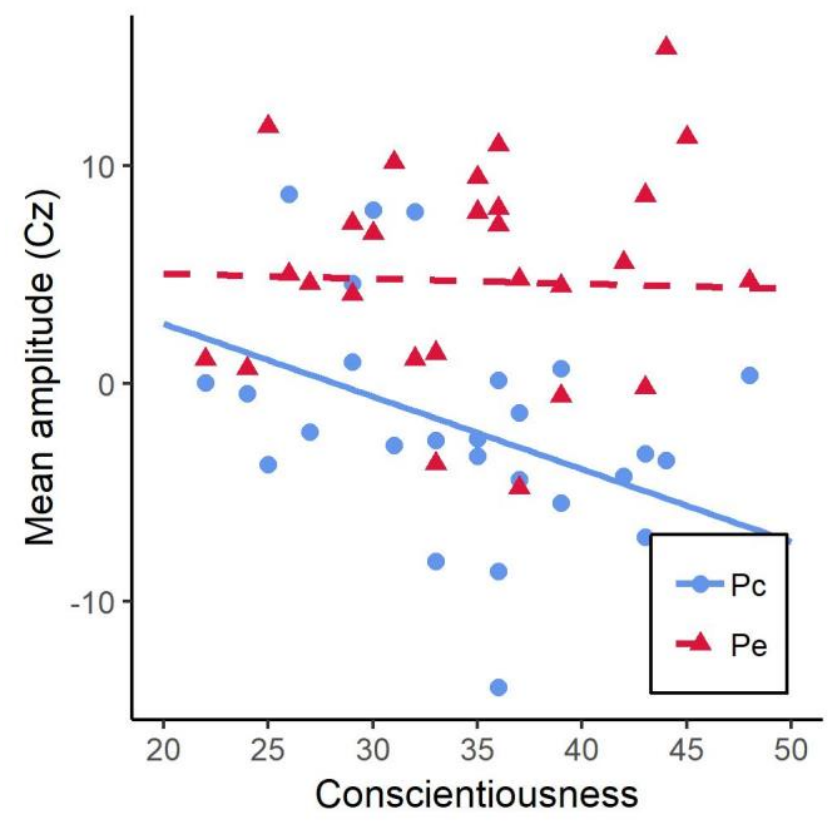

Figure 8. Mean amplitudes during 200-350ms post-response interval at electrode $\mathrm{Cz}$ as a function of conscientiousness for correct $(\mathrm{Pc})$ and incorrect responses $(\mathrm{Pe})$. The lines resulted from regressing Pc and Pe amplitudes onto conscientiousness.

I ran two separate regression analyses examining the influence of conscientiousness on both the Pe and Pc amplitude to elucidate the significant interaction of conscientiousness and accuracy. Conscientiousness was found to be a significant predictor of $\mathrm{Pc}$ amplitude, $\beta=-.44, t(26)=-2.52, p<.018$, but not of Pe amplitude, $\beta=-.03, t(26)=-0.14, p=.89$. The regression lines are depicted in Figure 8 . This indicated that lower values of conscientiousness were associated with higher Pc amplitudes. 
$\underline{\text { Performance monitoring and correct response significance in conscientious individuals }}$

\subsubsection{Analysis of CRN and Pc with larger sample}

To gain more power for the analysis of the CRN, I conducted additional analyses exclusively for $\mathrm{CRN}$ and $\mathrm{Pc}$ amplitudes with a larger dataset including participants that were sorted out before due to insufficient numbers of artifact-free error trials.

Again I conducted a linear mixed-effects regression analysis with fixed effects rule condition and conscientiousness (including interaction term) to examine the influence of conscientiousness during rule conditions on the CRN. The main effect of conscientiousness approached significance, $F(1,43)=3.16, p=.08$, whereas other effects remained nonsignificant, $F \mathrm{~s}<2.14, p \mathrm{~s}>.12$. These results confirmed the previous indication that there might be an association of conscientiousness and the CRN amplitude.

Another linear mixed-effects regression analysis with fixed effects rule condition and conscientiousness (including interaction term) was computed to investigate whether there was an influence of conscientiousness and rule instructions on the Pc amplitude. A significant main effect of conscientiousness was revealed, $F(1,43)=5.51, p=.024$. The main effect of rule condition was found to be significant, $F(2,86)=3.79, p=.027$. Bonferroni post hoc tests used to break down this main effect of rule condition revealed that instruction from STD condition evoked a lower Pc amplitude than instructions from RR $(\mathrm{p}<$ $.001)$ and $\mathrm{RV}(\mathrm{p}<.001)$.

For the purpose of illustration low $(M=29.59, S D=4.12)$ and high $(M=41$, $S D=3.79)$ conscientiousness groups were formed using a median split procedure. The response-locked ERP averages of trials with correct responses separated by conscientiousness groups (low or high) are depicted in Figure 9. Topographical maps of CRN and Pc are illustrated in Figure 10 and Figure 11. 

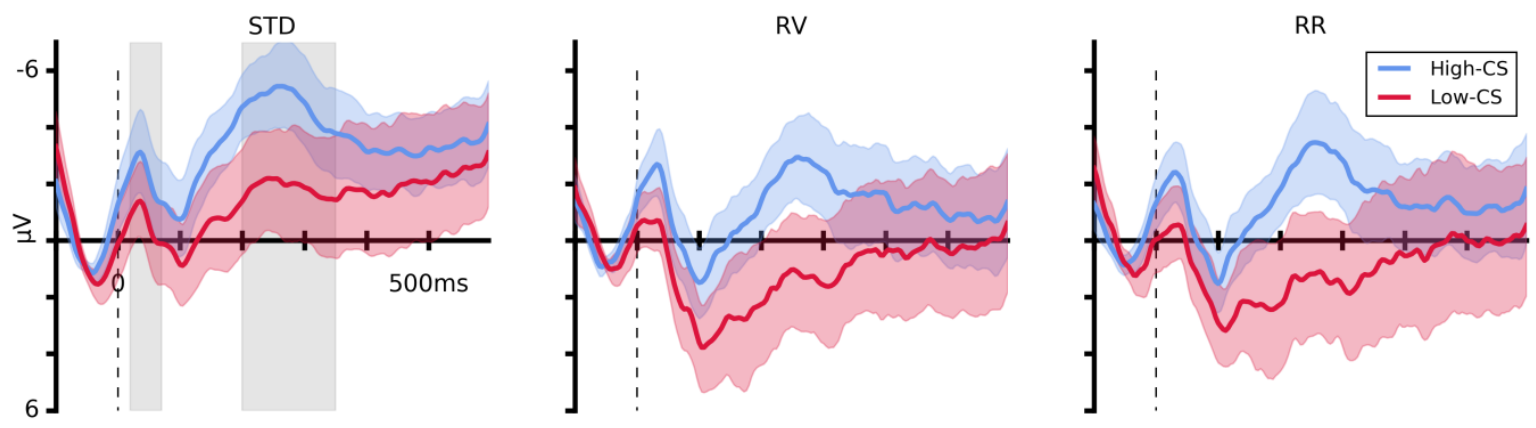

Figure 9. Response-locked ERPs for correct responses at electrode $\mathrm{FCz}$ for both conscientiousness (CS) groups separated by instruction conditions. The three panels show the ERPs of standard (STD), rule violation (RV), and rule reversal (RR) conditions, respectively. Color-shaded areas indicate the $95 \%$ confidence intervals for the mean ERPs. Gray-shaded areas mark the time windows of CRN and Pc.
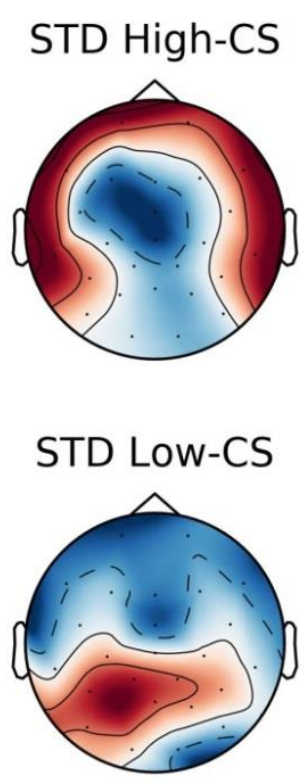
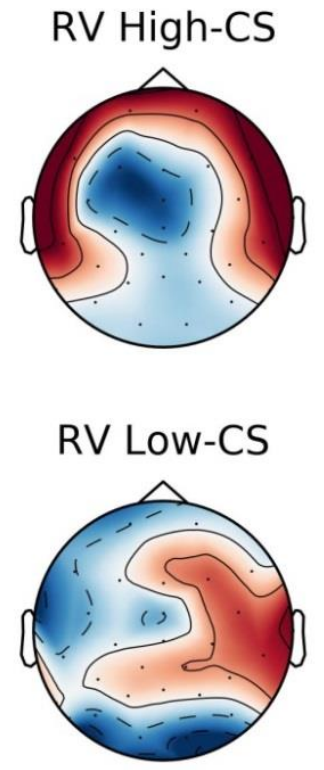
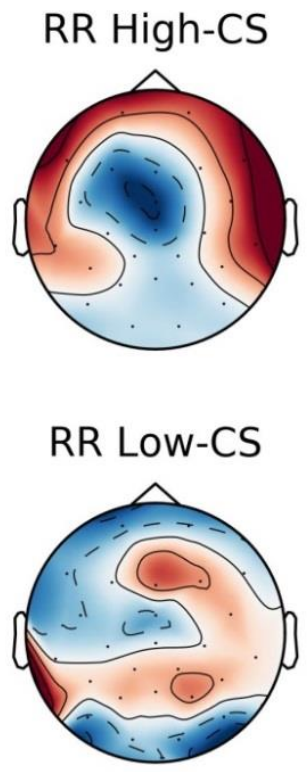

Z

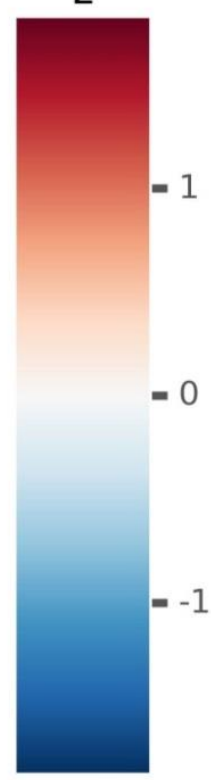

Figure 10. Z-transformed voltage distributions during response-locked ERP after correct responses in a time window of $30-50 \mathrm{~ms}$. The upper and lower heads show distributions of participants scoring high and low on conscientiousness (CS), respectively. The distributions of each group are displayed separately by column for the instruction conditions standard (STD), rule violation (RV) and rule reversal (RR). 

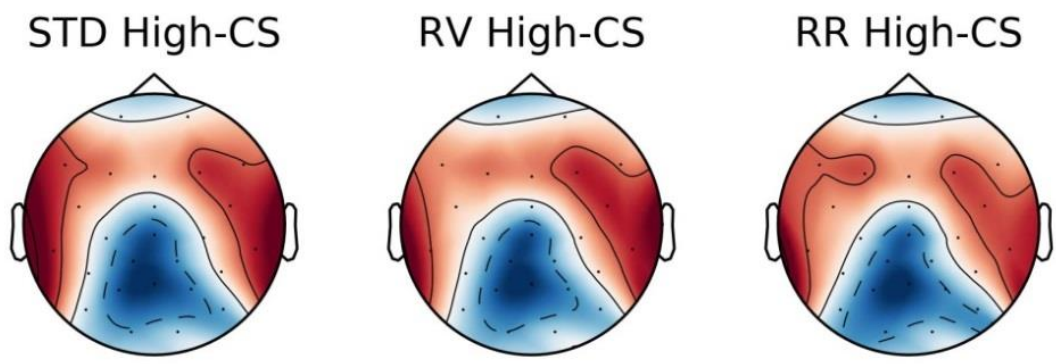

Z
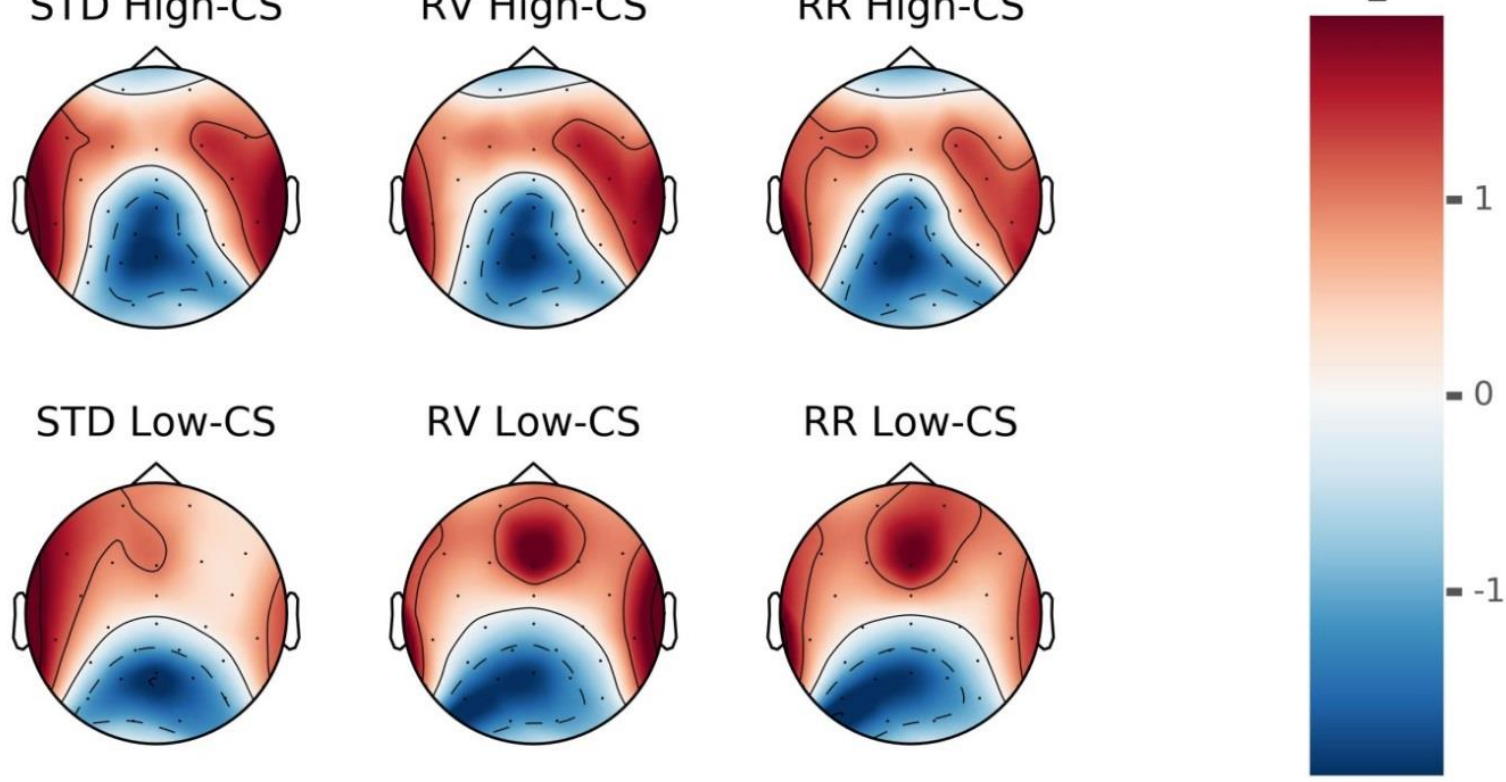

Figure 11. Z-transformed voltage distributions during response-locked ERP after correct responses in a time window of $200-300 \mathrm{~ms}$. The upper and lower heads show distributions of participants scoring high and low on conscientiousness (CS), respectively. The distributions of each group are displayed separately by column for the instruction conditions standard (STD), rule violation (RV) and rule reversal (RR). 
$\underline{\text { Performance monitoring and correct response significance in conscientious individuals }}$

\subsection{Discussion}

The main purpose of Experiment 1 was to examine the variability of ERN and CRNamplitude as a function of conscientiousness and rule violation behavior. For this purpose, I designed a choice reaction task with simple rules consisting of responding to a circle and a square by pressing the left and the right key, respectively. In two other conditions, participants were asked to either reverse or violate the instructed rules. I assumed that conscientiousness is associated with better task performance (displayed by lower error rates and shortened RTs) but higher cognitive conflict (displayed by prolonged post-error slowing) as well as increased ERN and CRN. Rule violations were expected to be accompanied by prolonged RTs and an increase in ERN and CRN.

The results of the present study partly confirmed these hypotheses. There are weak signs of better task performance for more conscientious individuals which manifested in a tendency of lower error rates but not shorter RTs. I observed an electrophysiological signature that was associated with conscientiousness. An interaction of conscientiousness and accuracy showed that the $\triangle \mathrm{ERN}$ was negatively related to conscientiousness. This pattern descriptively manifested by both a positive relationship of CRN magnitude with conscientiousness and a negative relationship of ERN amplitude with conscientiousness. Electrophysiological differences were also revealed by an association of conscientiousness and the magnitude of the Pc. Lower values on the conscientiousness scale were associated with higher amplitudes of the Pc. In addition, comparing RV and RR trials to STD trials, participants were showing a lower amplitude of CRN and a more pronounced amplitude of Pc. Opposed to my hypotheses, the instruction to violate the rules had the same effect as simple task switches on both behavioral performance, and response-locked ERPs at medialfrontal electrodes. There was also no measurable influence of conscientiousness on these effects.

It should be noted that all findings regarding the ERN should be interpreted with caution due to the relatively small number of errors generated overall, $M=5.5 \%, S E=.38$. This might be a result of the experimental design. After artefact rejection only few trials were left for analysis of variations in ERN amplitude. I only included participants with at 
$\underline{\text { Performance monitoring and correct response significance in conscientious individuals }}$

least 5 error trials in all analyses (except for particular analyses concerning CRN/Pc where only correct trials were needed). However, it is recommended to have a minimal trial number of 15 for within-subject comparisons in experiments using the ERN as dependent variable (Fischer, Klein, \& Ullsperger, 2017). Future research may aim at altering the experimental design so that participants are forced to commit more errors.

\subsubsection{Higher task engagement in conscientious individu- als}

The weak association of lower error rates and high conscientiousness might indicate a different task performance strategy which may be affected by a greater concern over errors and a higher motivation to respond correctly. However, this interpretation is rather speculative since there was only a tendency of lower error rates in high conscientious individuals. This result pattern might indicate that participants scoring high on the conscientiousness scale made a greater effort to respond correctly in contrast to individuals with lower levels of conscientiousness. More conscientious individuals may therefore have a stronger focus on task performance.

The ERP findings would support the interpretations drawn from the behavioral data concerning task performance strategies. Most intriguingly, there was an inverse relationship of the amplitudes of ERN and CRN depending on conscientiousness. With an increase of conscientiousness, the amplitude of ERN decreased while the magnitude of CRN enhanced. This was also expressed in a decrease of the $\triangle E R N$ when conscientiousness increased. This observation in combination with better accuracy values of more conscientious individuals, is in line with the assumption that CRN reflects task engagement (Simons, 2010). It also supports the notion that individuals with high values on the conscientiousness scale are characterized by stronger task engagement and stronger focus on task performance. Additionally, for participants high in conscientiousness, the indicated pattern of enhanced CRN amplitude associated with lower error rates is pointing in the same direction as findings that CRN magnitude is inversely related to the probability of committing errors (Allain et al., 2004). Allain and colleagues believe that larger CRN amplitudes could have a preventive function against committing errors. Moreover, some other studies re- 
$\underline{\text { Performance monitoring and correct response significance in conscientious individuals }}$

ported similar patterns providing further evidence for the interpretation of different task performance strategies. For example, a recent study of Kalamala and colleagues (2018) found enhanced ERN and CRN amplitudes as well as higher accuracy values in bilinguals suggesting that they are pursuing an accuracy-focused strategy. Hajcak and Simons (2002) also reported enhanced ERN and CRN in participants with obsessive-compulsive characteristics.

I therefore conclude that conscientiousness is associated with a heightened motivational salience of an error-free task completion. Following the idea that ERN is reflecting error significance (Hajcak, Moser et al., 2005), the CRN may reflect a "correct response significance" and I suggest that higher values of conscientiousness may be linked to a stronger motivation to respond correctly due to the absence of a more pronounced ERN.

There might be a possible confoundation with anxiety, especially apprehension/worry which is related to higher ERN amplitudes (for a review see Moser et al., 2013) and cannot be cleared in the present experiment due to a missing control for this trait. However, according to Moser and colleagues (2013) there is no clear relationship of anxiety with higher magnitudes of CRN. In contrast, our data suggest that conscientiousness is related to lower or equal ERN but a tendency to higher CRN amplitudes. Thus, the electrophysiological pattern related to conscientiousness seems to contradict the pattern related to anxiety.

Pailing and Segalowitz (2004) found that the ERN amplitude of individuals low in conscientiousness was sensitive to motivation-related changes and was increased when monetary rewards were added to the experimental manipulation. In addition, Hill et al. (2016) observed a sensitivity of the ERN amplitude to negative urgency at low levels of conscientiousness consisting of an increase of the ERN amplitude when negative urgency was increased. The results of the present experiment are in line with this pattern and confirmed that there is no general difference in the ERN magnitude across various levels of conscientiousness. It may even be possible that the higher $\triangle E R N$ amplitude (i.e. consisting of a somewhat higher ERN amplitude but also smaller CRN amplitude) observed at low levels of conscientiousness were due to differences in negative urgency. However, this possible association cannot be cleared because I did not control for this trait. 
$\underline{\text { Performance monitoring and correct response significance in conscientious individuals }}$

In addition to a CRN that is tended to be increased in conscientious participants, there was an association of reduced Pc amplitudes and high conscientious individuals. In past studies, trials preceding errors were found to be characterized by a similar positivity following the CRN (Allain et al., 2004; Hajcak, Nieuwenhuis et al., 2005). It was interpreted as disengagement of the ACC from the monitoring process (Ridderinkhof et al., 2003). Although our analysis was not exclusively concerned with trials preceding errors, the increased Pc in participants with low levels of conscientiousness in the present study could also be explained by a disengagement of the response monitoring process. This view was also supported by higher error rates in participants scoring low on the conscientiousness scale. A Pc has also been found in participants with high negative affect (Hajcak, McDonald, \& Simons, 2004). Hajcak and colleagues found that this post-response positivity was more pronounced in an infrequent task. In line with their observation, I also found that the Pc was more pronounced in the RV and RR conditions (the infrequent conditions). The Pc might reflect the expectation of the upcoming trial type or a preparation process that controls how to respond next. To examine this assumption, future experimental settings have to investigate the role of frequency of the trial types.

It is conceivable that the observed Pc is similar to the post-response positive activity after errors known as Pe usually occuring during an interval of 200-350ms following the erroneous response. According to the assumption that ERN and CRN represent in part an identical cognitive control process during response monitoring (Burle et al., 2008; Meckler et al., 2011; Suchan et al., 2007), Pe and Pc could both be associated with an identical process as well. With regard to errors, there are two approaches to explain the Pe. It could either reflect error awareness (Overbeek et al., 2005; Steinhauser \& Yeung, 2010) or confidence about response correctness (Boldt \& Yeung, 2015). Boldt and Yeung found that confidence about response correctness varied inversely as a function of Pe magnitude. According to the last perspective and transferred to correct responses, the present data indicate that individuals with low levels of conscientiousness are less confident about the response-correctness which could also be explained by task disengagement. 
$\underline{\text { Performance monitoring and correct response significance in conscientious individuals }}$

\subsubsection{Effects of rule violations}

I observed generally higher RTs and error rates in RV and RR trials indicating a greater response conflict in these conditions. The findings concerning rule violations are partly in line with previous research on rule violation behavior (Pfister, Wirth, Schwarz, Steinhauser et al., 2016; Wirth et al., 2016). However, RTs and error rates from the present study did not differ between rule violations and rule reversals which may imply that violating a rule does not differ from changing the rule with regard to the evoked cognitive conflict. The nonexistent effects of rule violations in the present experiment might be due to the withinsubject manipulation while effects found by Wirth and colleagues (2016) were based on a between-group design. Corresponding to the nonexistent effects of rule violations in behavioral data, I did not observe any differences in the response-locked ERPs between rule violations and rule reversals.

The behaviorally observed cognitive conflict during RV and RR trials (compared to STD trials) could be attributed to a concurrent activation of multiple competing responses. I argued that such a cognitive conflict could have an influence on the ERN or CRN amplitudes. However, according to my observations this conflict was not reflected by differences in ERN, supporting evidence that questions conflict monitoring approaches (Burle et al., 2008; Carbonnell \& Falkenstein, 2006; Masaki et al., 2007). On the contrary, there were equal CRN amplitudes in high conflict trials from RV and RR conditions compared to low conflict trials from STD condition although arguing from a conflict monitoring perspective one could expect higher CRN amplitudes.

A Pc-like component in correct rule violations was at least descriptively also found in the study of Pfister and colleagues (2016) but the authors left it without comment. However, the present findings suggests that the similarity of the waveform in both RV and RR trials indicates that this component may reflect a more general process related to rule modifications, not one specific to rule violations. 
$\underline{\text { Performance monitoring and correct response significance in conscientious individuals }}$

\subsubsection{Problems with the task design}

Although the task could highlight some differences in performance monitoring of conscientious individuals, it seems obvious that the experimental manipulation failed with respect to the initiation of rule violation behavior. Possible explanations could be the following. First, some participants reported that they internally translated instructions from both RV and RR condition to one instruction that required the same response. They obviously reframed the instructions and did not differentiate the meaning of violating the rule from reversing the rule. Second, a habituation effect could have occurred when violating the rule was instructed too often since $20 \%$ of all trials were rule violation trials. Lastly, the instruction to violate a rule may not have created an ecologically valid feeling of breaking a rule in participants at all. The explicit instruction to violate the rule might have had the effect of a legitimization to break the rule leading to the feeling of rule-compliant behavior instead.

These observations as well as the low error rate mentioned earlier, showed that the task was less than ideal. There are some crucial steps to improve the task. First, the implementation of an Eriksen flanker task (Eriksen \& Eriksen, 1974) might lead to a higher error rate. In contrast to the simple choice-reaction task employed in Experiment 1, the flanker task has been successfully utilized to achieve a suitable number of errors. Second, the experimental manipulation of rule violations should be based on a between-group design. Instead of instructing to violate and to reverse the rule in the same participant, both instructions should be given in different groups to prevent reframing of task instructions. These alterations in the task design may shed light upon whether there are effects of rule violations in choice-reaction tasks associated with conscientiousness.

\subsubsection{Limitations}

It has to be mentioned that all findings regarding conscientiousness should be interpreted with caution due to the distribution of conscientiousness values in the sample. The whole sample actually exhibited conscientiousness values above average corresponding to 
$\underline{\text { Performance monitoring and correct response significance in conscientious individuals }}$

actual moderate to high conscientiousness values. Future research should aim at observing response monitoring after obtaining a broader range of conscientiousness values.

\subsubsection{Conclusion}

To summarize, the present study provided first evidence that conscientiousness might be associated with the $\triangle \mathrm{ERN}$ amplitude. The data indicates that high conscientiousness is linked to a larger magnitude of the CRN and smaller amplitude of the ERN. The decrease of $\triangle \mathrm{ERN}$ with an increase in conscientiousness might be explained by a shift of motivational significance of errors towards a motivational significance of correct responses. This supports the perspective that increases in CRN amplitude might reflect task engagement and motivational salience of an error-free task completion, which can also be expressed as "correct-response significance". This perspective is further strengthened by higher Pc amplitudes in individuals having low levels of conscientiousness which has been linked to disengagement of the response monitoring. Furthermore, cognitive conflict during rule violations and rule reversals indicated by prolonged RTs and higher error rates was not reflected by differences in CRN or ERN. This observation contradicted conflict model accounts of the ERN. 


\section{The impact of conflict on performance monitoring in conscientious individu-} als

\subsection{Introduction}

Experiment 1 showed a relationship of conscientiousness with the response-locked ERP in a setting where participants are forced to switch between different tasks that consisted in responding according to a rule, reversing and violating the rule. In comparison with less conscientious individuals, individuals scoring high on a conscientiousness scale showed a tendency to lower error rates with equal response times as well as a decreased $\triangle$ ERN together with a tendency to higher CRN amplitudes and lower Pc amplitudes.

However, the assumption that rule violations have a response-locked electrophysiological signature consisting of an emphasis of ERN and CRN that might even be enhanced in more conscientious individuals was rejected. Although the required responses were the same in both conditions, there was neither a behavioral nor an electrophysiological difference between instructed task switches, which were in accordance with the rules, and those that aimed at violating the rules within an individual. There are possible reasons that might explain the missing difference between effects of violating a rule compared to rule-based task switches. Participants might not have differentiated between both conditions because they internally reframed the instructions to an instruction meaning the same. This might be due to the fact that both instructions actually required the same response.

The results from Experiment 1 indicated a relationship of conscientiousness and an enhanced CRN which is in line with assumptions that CRN reflects task engagement (Si- 
The impact of conflict on performance monitoring in conscientious individuals

mons, 2010). The results however did not indicate a relationship of conscientiousness and the ERN amplitude. This pattern is not entirely in line with current research on the ERN suggesting that a reduced ERN is related to motivational disengagement, disinhibition and decreased conscientiousness, whereas an enhanced ERN is associated with perfectionism, concern over errors, negative affect and intolerance with uncertainty (Weinberg et al., 2012). A reason why these assumptions were not confirmed might be that Experiment 1 faced methodological problems. The overall low error rate lead to an insufficient number of errors, hence it was not possible to reliably compare the ERN amplitude between individuals scoring low and high on a conscientiousness scale. Additionally, the sample was characterized by a skewed distribution of conscientiousness with actual conscientiousness values above average. Therefore, results concerning the electrophysiological correlates of conscientiousness must be interpreted with caution.

Experiment 2 aims at overcoming these methodological problems. As in Experiment 1 I want to examine the effects of conscientiousness and rule violations on the medial-frontal response-locked ERPs in both errors and correct responses.

For this purpose, an adopted Eriksen Flanker Task (Eriksen \& Eriksen, 1974) was utilized. Target and surrounding (flanker) stimuli were triangles. Participants had to respond to a "<" (i.e. a triangle pointing to the left) and a ">" (i.e. a triangle pointing to the right) by pressing the left and right key, respectively. Surrounding stimuli were also triangles ordered vertically and pointed either to the same or to the opposite direction. Following in part the design from Experiment 1, the experiment consisted of two instruction conditions. During one condition participants were asked to respond according to the rule just described (corresponding to the STD condition). During the second condition they were instructed to either violate the rule (RV) or reverse the rule (RR). The significant difference to Experiment 1 was that the instruction given during the second condition varied dependent on the group the participants were assigned to prior to the experiment. Accordingly, participants were assigned to either RV or RR group to prevent that subjects internally translate both conditions to the same meaning. It is particularly significant that the S-R mapping required the same responses in both RV and RR group during all conditions. 
The impact of conflict on performance monitoring in conscientious individuals

Another alteration to Experiment 1 was constituted by the selection process of participants. Instead of selecting a sample and performing a median split afterwards, a comparison of extreme groups was performed. A sample of 132 individuals was screened using the NEO Five Factor Inventory (NEO-FFI; Borkenau \& Ostendorf, 2008) for this purpose. Then, two groups with participants scoring low and high on the NEO-FFI conscientiousness scale were formed ensuring a maximal possible distance between both groups. This procedure should allow for a clearer distinction of features that are associated with conscientiousness.

I expected prolonged RTs and higher error rates for rule violations compared to the rule-based behavior. The response-locked ERP at medial-frontal positions is assumed to show a pronounced negativity in a $100 \mathrm{~ms}$ post-response interval (corresponding to ERN and $\mathrm{CRN}$ ). On basis of observations in Experiment 1, no difference in RTs between both conscientiousness groups but lower error rates in participants with high conscientiousness values are expected. The medial-frontal response-locked ERP during an interval of $100 \mathrm{~ms}$ after the response is presumably more pronounced in conscientious individuals. It is also assumed that there is an increasing effect of congruency on RTs, error rates and negativity during $100 \mathrm{~ms}$ post-response time window in response-locked ERP at medial-frontal electrodes. 


\subsection{Materials and Methods}

\subsubsection{Participants}

54 participants ( 8 male, 6 left-handed) between the ages of 18 and 38 years $(M=20.93$, $S D=3.54)$ were recruited and received $3-\mathrm{hr}$ course credit. The sample was selected on basis of a pre-assessment of 132 mainly undergraduate university students of Psychology in lectures and seminars by means of the NEO-FFI. All participants of the pre-assessment received 0.5 -hr course credit. Participants were selected on basis of the conscientiousness scale $(M=51.05, S D=12.03$, Min $=23, \operatorname{Max}=80)$. I selected participants with conscientiousness scores that deviated by at least 0.8 standard deviations in both directions from the total mean of the normative sample on the conscientiousness scale. As a result I obtained two equally-sized groups ( $n=27$ each) with participants scoring low and high on the conscientiousness scale. All participants were free of neurological disorders (e.g. epilepsy) and had normal or corrected-to-normal visual ability. All participants were naive concerning the hypothesis underlying the experiment. The participants were informed that they could withdraw from the study at any time without penalty or loss of privilege.

\subsubsection{Materials}

\subsubsection{Questionnaires}

The NEO-FFI (Borkenau \& Ostendorf, 2008; Costa \& McCrae, 1992) was used for pre-assessment and sample selection. The same version had been used for experiment 1 (see chapter 2). In addition, the three subscales order, dutifulness and achievement striving from the conscientiousness scale of the Revised NEO Personality Inventory (NEO-PI-R; Costa \& McCrae, 1992; Ostendorf \& Angleitner, 2004) as well as the conscientiousness scale of the Bochum Inventory for profession-related Personality description (BIP; Bochumer Inventar zur berufsbezogenen Persönlichkeitsbeurteilung; Hossiep, Paschen, \& Mühlhaus, 2003) were administered.

The NEO-PI-R measures the same Five Factor Model of personality (McCrae \& Costa, 1990) as the NEO-FFI which is the shorter version. The whole questionnaire consists of 
The impact of conflict on performance monitoring in conscientious individuals

240 items that aim at measuring the five domains conscientiousness, extraversion, agreeableness, neuroticism, and openness. Each domain consists of 6 subscales with 8 items each.

The BIP aims at measuring personality traits in the profession-related context. It consists of 14 scales divided into 4 domains work behavior, professional orientation, social skills, and mental constitution. The scale conscientiousness belongs to work behavior and consists of 14 items.

\subsubsection{Stimuli and Apparatus}

Stimuli were displayed on a 24" screen with a resolution of 1600 x 900 pixels. Participants had an average viewing distance of $65 \mathrm{~cm}$. Stimuli were based on Eriksen Flanker Task (Eriksen \& Eriksen, 1974) and are listed in Table 4. They consisted of five triangles that were ordered vertically. Each triangle measured $8 \mathrm{~mm}$ corresponding to a display size of $0.71^{\circ}$ of visual angle. The distance between triangles were $6 \mathrm{~mm}$ corresponding to a display size of $0.52^{\circ}$ of visual angle. Two types of stimuli determined either (a) the stimulus was congruent, i.e. target (central) and surrounding (flanker) stimuli point to the same direction, or (b) the stimulus is incongruent, i.e. target and surrounding stimuli point to opposite directions.

Table 4. Four types of stimuli categories dependent on congruency of flanker arrows (congruent or incongruent) and direction of target arrows (left or right)

\begin{tabular}{lcccc}
\hline Target & Right & Right & Left & Left \\
\hline Type & Congruent & Incongruent & Congruent & Incongruent \\
\hline Stimuli & & & & \\
& & & &
\end{tabular}

A button box (Cedrus RB-830, San Pedro, CA) served as response device that had one button for each left and right responses. Participants were instructed to respond bimanually by using the index finger of each hand to press the buttons. Presentation of the instructions and stimuli in a randomized order was controlled by Presentation Version 16 (Neurobehav- 
The impact of conflict on performance monitoring in conscientious individuals

ioral Systems, Inc. Albany, CA). Participants were asked to respond to the target triangle according to the overall rule that required pressing the left button when the triangle points to the left and pressing the right button when the triangle points to the right.

\subsubsection{Procedure}

An exemplary trial is depicted in Figure 12. The trial procedure began with the presentation of a blank screen $(1000 \mathrm{~ms})$. Then, an instruction text $(1000 \mathrm{~ms})$ according to one of two conditions followed and a fixation cross was presented $(500 \mathrm{~ms})$. After that, the flanker stimuli were presented $(50 \mathrm{~ms})$ and finally the display of the target stimulus completed the trial presentation. Flanker and target stimuli remained on screen until the participant responded. 
$1000 \mathrm{~ms}$
blank screen

$1000 \mathrm{~ms}$

instruction

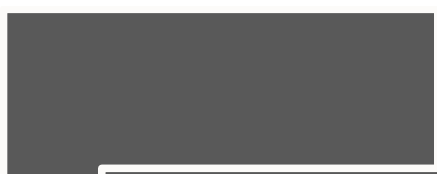

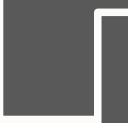
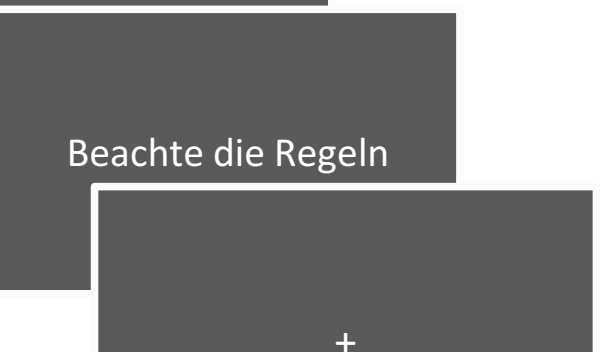

$500 \mathrm{~ms}$

\section{fixation}

$50 \mathrm{~ms}$
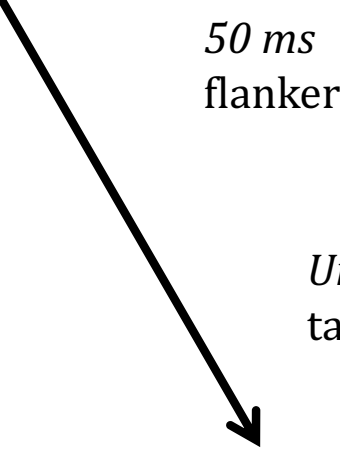

Until response target

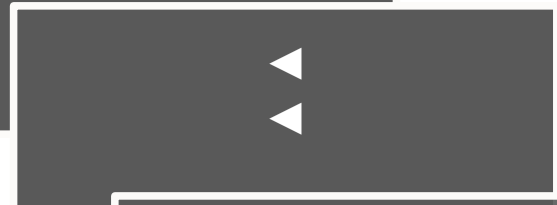

Figure 12. Procedure of a trial. The following screens were presented in the order they are mentioned including their presentation durations in the following: blank screen $(1000 \mathrm{~ms})$, instruction $(500 \mathrm{~ms})$, fixation cross $(500 \mathrm{~ms})$, flanker stimuli $(50 \mathrm{~ms})$ and flanker stimuli combined with target stimulus (until response).

Participants completed 30 blocks of 20 trials (600 trials in total) and blocks were separated by breaks whose length could be determined by the participants themselves. A preceding training block of 20 trials was considered for practice and excluded from data analysis.

The experiment consisted of $80 \%$ trials from standard (STD) instruction condition and $20 \%$ from an alternative (ALT) instruction condition. All trials were presented in a randomized order across the experiment. The flanker stimuli types (150 each) were equally distributed over both STD and ALT condition. 
The impact of conflict on performance monitoring in conscientious individuals

At the beginning of the experiment, it was explained to the participants that there is an overarching rule that requires pressing the left button when the target triangle points to the left and pressing the right button when the target triangle points to the right. During trials from the standard condition, participants were instructed to respond according to the overarching rule by means of displaying the instruction "Beachte die Regeln" (German for "Mind the rules"). During trials of ALT condition the instruction changed. Depending on the assigned instruction group, participants were instructed to either violate the rule by means of displaying the instruction "Mache einen Fehler" (German for "Commit an error") or reverse the rules by means of displaying the instruction "Vertausche die Regeln" (German for "Reverse the rules"). To summarize, there were the following three types of instruction conditions, depending on the condition the target stimuli and their corresponding correct responses were:

- Standard (STD): "<" - left button, ">" - right button

- Alternative (ALT) instruction conditions:

○ Rule violation (RV): “<” - right button, ">” - left button

○ Rule reversal (RR): “ "<" - right button, ">” - left button

As described in Chapter 3.2.1 I assigned pre-assessed participants to two groups with low and high values of conscientiousness. Both groups were further subdivided into two instruction groups which had different instructions during the ALT instruction condition mentioned above. Parallel distribution of conscientiousness in alternative instruction subgroups for both low and high conscientiousness was carefully ensured. Table 4 gives an overview of how many participants were assigned to the four groups that differ in terms of their scores on the conscientiousness scale and instruction during the ALT condition.

Subsequent to the experiment, participants were asked to complete the scales from the NEO-PI-R (Costa \& McCrae, 1992) as well as BIP (Hossiep et al., 2003) that were mentioned in chapter 3.2.2.1. 
The impact of conflict on performance monitoring in conscientious individuals

Table 5. Subdivision of the sample in terms of expression on conscientiousness scale and instruction during alternative instruction condition

\begin{tabular}{lcccccc}
\hline \multirow{2}{*}{$\begin{array}{l}\text { Group } \\
\text { Conscientiousness }\end{array}$} & \multicolumn{2}{c}{ Rule Violation } & & \multicolumn{2}{c}{ Rule Reversal } \\
\cline { 2 - 3 } \cline { 5 - 6 } $\mathrm{N}$ & High & Low & & High & Low \\
\cline { 2 - 3 } \cline { 5 - 6 } & 15 & 12 & & 15 & 12 \\
\hline
\end{tabular}

\subsubsection{EEG recordings and data analysis}

EEG data was recorded as in Experiment 1 (see chapter 2.2.4). However, only recorded data from electrodes $\mathrm{Fz}, \mathrm{FCz}, \mathrm{Cz}$ and $\mathrm{Pz}$ were used for statistical analysis.

Subsequent to the recordings, EEG data was further prepared by using BrainVision Analyzer 2.0.1 (Brain Products GmbH, Germany). Exclusively trials with responses between $200 \mathrm{~ms}$ and $1500 \mathrm{~ms}$ after stimulus presentation were considered for analysis. All trials containing artifacts of eye movements were corrected using a blind component separation (Joyce et al., 2004). This procedure has been shown to be better than other artifact correction procedures (Kierkels et al., 2006).

All recordings were divided into equally-sized response-locked epochs for purpose of data analysis. Epochs starting $100 \mathrm{~ms}$ before and ending $600 \mathrm{~ms}$ after the response with a $100 \mathrm{~ms}$ pre-response baseline were formed. In addition, epochs with voltage steps of $20 \mu \mathrm{V} / \mathrm{ms}$ or differences of $300 \mu \mathrm{V}$ within an interval of $150 \mathrm{~ms}$ on each channel were rejected after ICA correction from further data analysis.

For statistical analysis of ERP data, I used the error-related components ERN and Pe as well as the CRN and Pc as counterparts on correct responses. Depending on the electrophysiological properties of the present ERP data, the ERN/CRN is measured as the mean amplitude during 0-70ms post-response interval at electrode FCz. The Pe was calculated as the mean amplitude during $150-350 \mathrm{~ms}$, the Pc as the mean amplitude during $80-150 \mathrm{~ms}$ post-response interval at electrode $\mathrm{Cz}$.

To include the three NEO-PI-R subscales order, dutifulness and achievement striving as well as the NEO-FFI conscientiousness scale and the BIP conscientiousness scale, I 
built a composite variable by averaging the $\mathrm{z}$ scores of these variables. This composite variable was used as conscientiousness measure for all statistical analyses.

Data for RT analysis was corrected for outliers by removing trials with RTs deviating more than 2.5 SDs from the mean RT of each participant and condition combination separately. PES is assessed in the same way as described in chapter 2.2.4.

I computed linear mixed-effects regression analyses using the lme4 package in $\mathrm{R}$ (Version 3.5.1; Bates et al., 2014) to assess effects of conscientiousness (continuous), rule group (RV vs. RR), instruction condition (STD vs. ALT), and congruency (congruent vs. incongruent) as well as their interactions on response-locked ERPs and behavioral measures error rate and response time. P-values for all models were calculated using the anova function of the lmerTest package (Kuznetsova et al., 2017) with a Kenward-Roger approximation of degrees of freedom. As random effects, intercepts for subjects and bysubject random slopes for the effects of conscientiousness, instruction condition and congruency were used.

Due to an overall low error rate, statistical analyses concentrate on the response-locked ERP components after correct responses, i.e. CRN and Pc. Otherwise, in case of taking erroneous responses into account, 49 participants would have been excluded (91\%) from all analyses due to commission of too few errors $(<5)$ in either of instruction conditions (STD, ALT) and congruency conditions (congruent, incongruent). 


\subsection{Results}

This section is divided into analysis of behavioral data and electrophysiological data.

\subsubsection{NEO and BIP scales}

The selected sample's correlations among NEO-FFI conscientiousness scale, selected NEO-PI-R conscientiousness subscales order, dutifulness, and achievement striving as well as BIP conscientiousness scale are provided in Table 6. Figure 13 shows the distribution of conscientiousness that was obtained from the sample. Sufficient ranges in conscientiousness t-scores $(\operatorname{Min}=23, \operatorname{Max}=80, M D=61, M=51.83, S D=15.85)$ with possible ranges of 0 to 100 were achieved.

Internal consistencies measured by cronbach's alpha of NEO-FFI conscientiousness scale was $\alpha=.84$, of the NEO-PI-R conscientiousness subscales order $\alpha=.66$, dutifulness $\alpha=.62$ and achievement striving $\alpha=.67$, as well as of the BIP conscientiousness scale $\alpha=.83$.

Table 6. Correlations among conscientiousness scales measured with NEO-FFI, subscales of NEOPI-R, and BIP.

\begin{tabular}{|c|c|c|c|c|}
\hline Personality scale & Order & Dutifulness & $\begin{array}{c}\text { Achieve- } \\
\text { ment } \\
\text { striving }\end{array}$ & $\begin{array}{l}\text { BIP consci- } \\
\text { entiousness }\end{array}$ \\
\hline \multicolumn{5}{|l|}{ NEO-FFI scale } \\
\hline Conscientiousness & $.81 * * *$ & $.65 * * *$ & $.74 * * *$ & $-.83 * * *$ \\
\hline \multicolumn{5}{|c|}{$\begin{array}{l}\text { NEO-PI-R } \\
\text { conscientiousness subscales }\end{array}$} \\
\hline Order & & $.70 * * *$ & $.63 * * *$ & $-.80 * * *$ \\
\hline Dutifulness & & & $.55 * * *$ & $-.57 * * *$ \\
\hline Achievement striving & & & & $-.70 * * *$ \\
\hline \multicolumn{5}{|l|}{ BIP } \\
\hline Conscientiousness & & & & \\
\hline
\end{tabular}




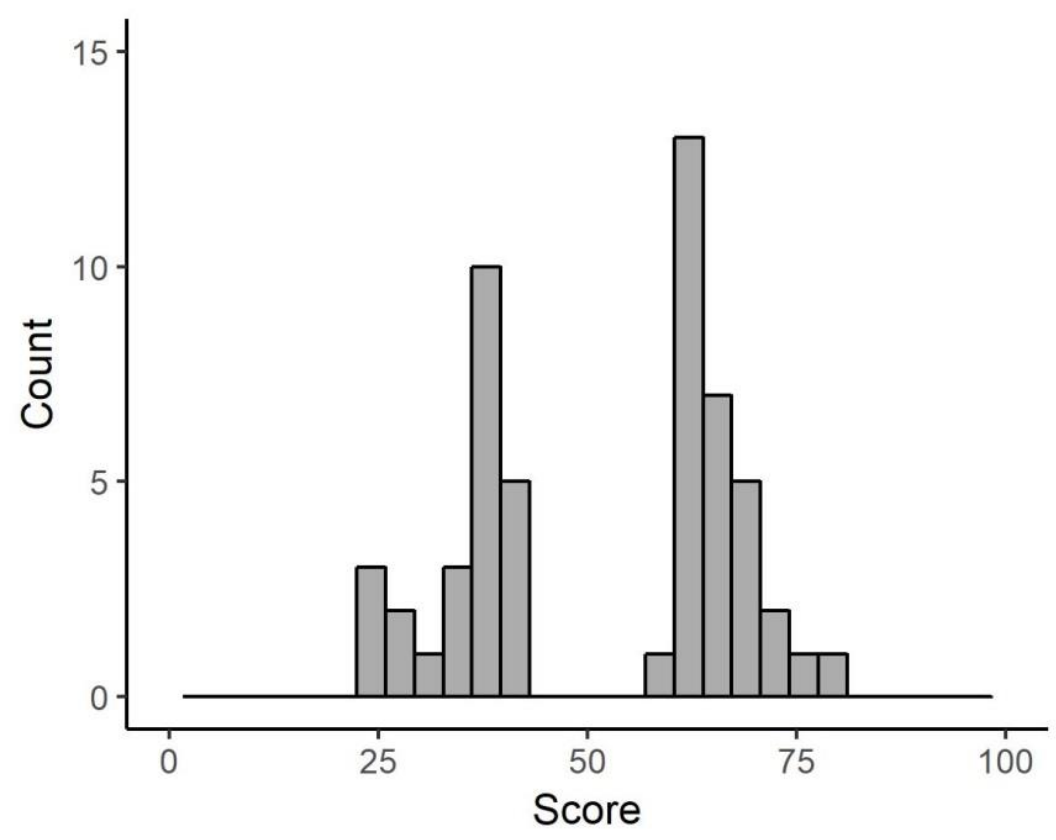

Figure 13. Distribution of conscientiousness t-scores obtained from sample of Experiment 2.

\subsubsection{Behavioral data}

For analysis of behavioral data, a series of ANOVAs for the dependent variables error rate, RT and PES were computed.

\subsubsection{Error rates}

Mean error rates (expressed as percentages) are illustrated in Figure 14. They were compared utilizing a linear mixed-effects regression model with between-group factors conscientiousness (continuous) and rule group (RV vs. RR), as well as repeated measures factors instruction condition (STD vs. ALT) and congruency (congruent vs. incongruent). 


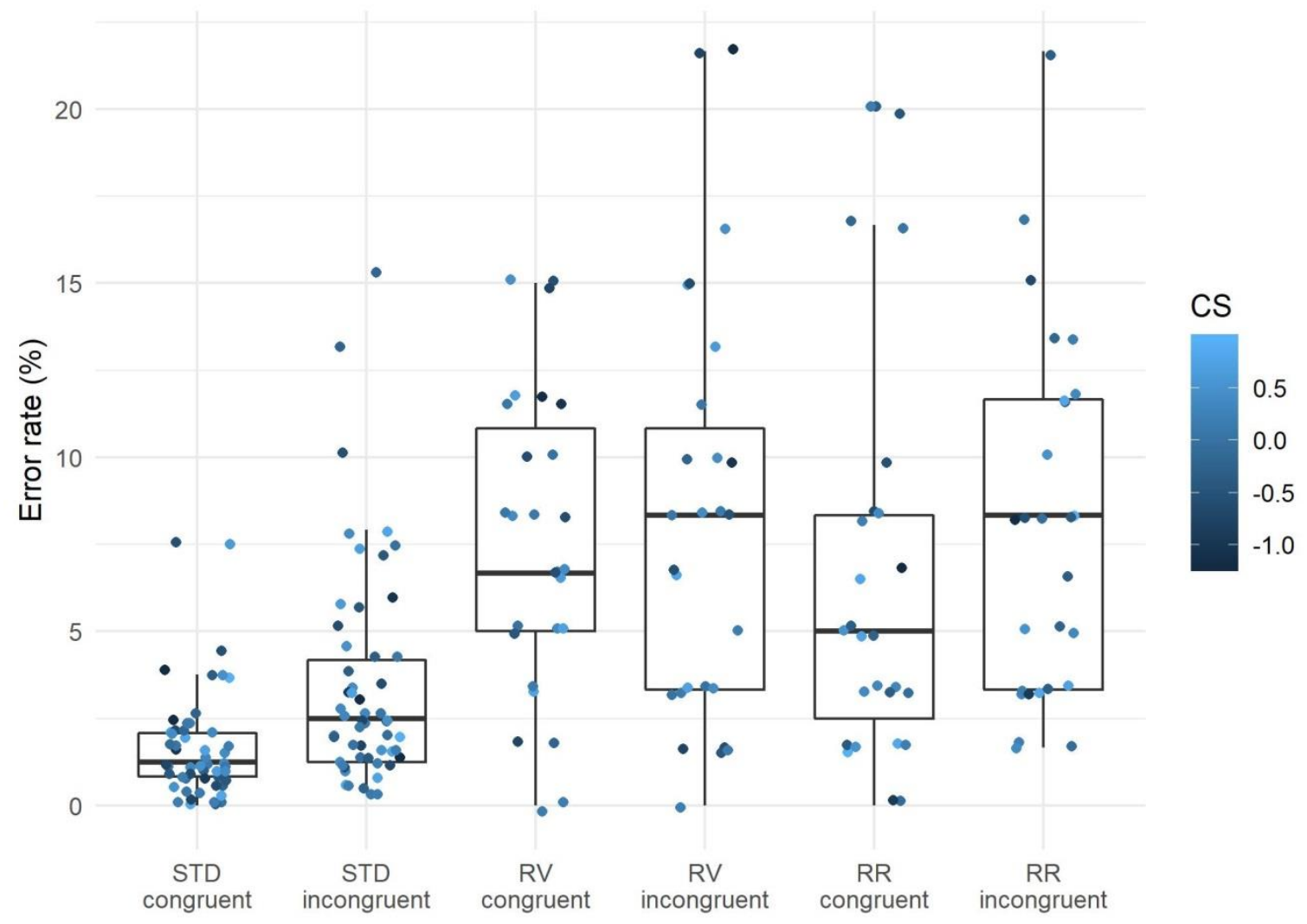

Figure 14. Mean error rates are displayed separately for each instruction condition, standard (STD), rule violation (RV), and rule reversal (RR) splitted by congruency condition (congruent, incongruent). Scatter plots overlaying each boxplot display the error rate as a function of conscientiousness (CS), the extent of which is represented by color intensity (high CS values are represented by low color intensity).

The analysis revealed a significant main effect of congruency, $F(1,50)=10.45$, $p=.002$, with subjects having higher error rates in incongruent flanker trials $(M=5.73$, $S E=0.49)$ than in congruent flanker trials $(M=4.42, S E=0.46)$. There was also a highly significant main effect of instruction condition, $F(1,50)=60.70, p<.001$, with participants having lower error rates in STD condition $(M=2.63, S E=0.25)$ than in the ALT condition $(M=7.61, S E=0.53)$. The interaction between the factors rule group and instruction condition was non-significant, $F(1,50)=0.01, p=.916$. This indicated that there was no difference between violating and reversing the rules in error rates. All other effects remained non-significant, $F_{\mathrm{S}}<2.60, p \mathrm{~s}>.113$. Inferential statistics and other effects are described in detail in Table 7. 
The impact of conflict on performance monitoring in conscientious individuals

Table 7. Statistics of the linear mixed-effects regression model with error rate as dependent variable.

\begin{tabular}{lccc}
\hline & $d f_{1}, d f_{2}$ & $F$ & $p$ \\
\hline CS & 1,50 & 2.60 & .113 \\
Rule group & 1,50 & 0.05 & .826 \\
Instruction condition & 1,50 & $60.70^{* * *}$ & $<.001$ \\
Congruency & 1,50 & $10.45^{* *}$ & .002 \\
CS x Rule group & 1,50 & 0.06 & .811 \\
CS x Instruction condition & 1,50 & 2.37 & .130 \\
Rule group x Instruction condition & 1,50 & 0.01 & .916 \\
CS x Congruency & 1,50 & 0.00 & .947 \\
Rule group x Congruency & 1,50 & 0.02 & .889 \\
Instruction condition x Congruency & 1,50 & 1.85 & .180 \\
CS x Rule group x Instruction condition & 1,50 & 0.11 & .744 \\
CS x Rule group x Congruency & 1,50 & 0.26 & .615 \\
CS x Instruction condition x Congruency & 1,50 & 0.25 & .619 \\
Rule group x Instruction condition x Congruency & 1,50 & 0.33 & .566 \\
CS x Rule group x Instruction condition x Congruency & 1,50 & 0.01 & .922 \\
\hline
\end{tabular}

Note. $\mathrm{CS}=$ conscientiousness, $d f=$ degrees of freedom; $F=\mathrm{F}$-value; $p=$ p-value

\subsubsection{Response times}

Mean RTs (in ms) are depicted in Figure 15. To analyze differences in RTs, a linear mixed-effects regression model with between-group factors conscientiousness (continuous) and rule group (RV vs. RR), as well as repeated measures factors instruction condition (STD vs. ALT) and congruency (congruent vs. incongruent) was computed. 


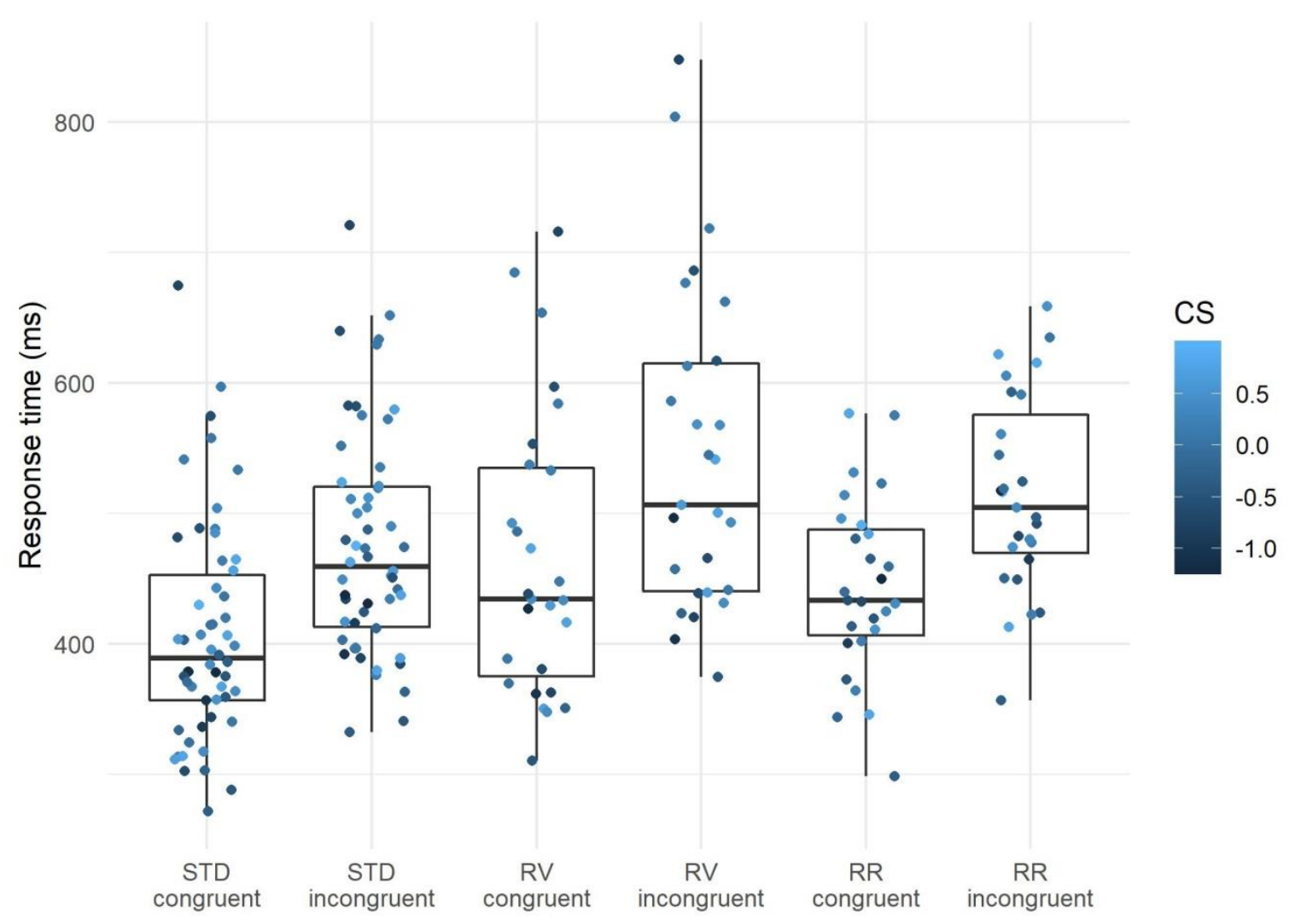

Figure 15. Mean response times are displayed separately for each instruction condition, standard (STD), rule violation (RV), and rule reversal (RR) splitted by congruency condition (congruent, incongruent). Scatter plots overlaying each boxplot display the response time as a function of conscientiousness (CS), the extent of which is represented by color intensity (high CS values are represented by low color intensity).

It revealed a highly significant main effect of congruency, $F(1,50)=426.58, p<.001$, with participants responding faster in the congruent condition ( $M=430 \mathrm{~ms}, S E=9 \mathrm{~ms})$ than in the incongruent condition ( $M=503 \mathrm{~ms}, S E=9 \mathrm{~ms})$. And there was a highly significant main effect of instruction condition, $F(1,50)=125.33, p<.001$, with subjects responding faster in STD condition $(M=441 \mathrm{~ms}, S E=9 \mathrm{~ms})$ than in ALT condition $(M=492 \mathrm{~ms}$, $S E=10 \mathrm{~ms})$. The interaction of factors instruction condition and congruency approached significance, $F(1,50)=3.46, p=.069$. The interaction of the factors rule group and instruction condition remained non-significant, $F(1,50)=0.08, p=.785$ indicating that there was no difference between violating and reversing the rules in RTs. All other effects remained non-significant, $F \mathrm{~s}<1.46, p \mathrm{~s}>.233$, for a detailed description of inferential statistics and other effects see Table 8 . 
The impact of conflict on performance monitoring in conscientious individuals

Table 8. Statistics of the linear mixed-effects regression model with RT as dependent variable.

\begin{tabular}{lccc}
\hline & $d f_{1}, d f_{2}$ & $F$ & $p$ \\
\hline CS & 1,50 & 0.69 & .409 \\
Rule group & 1,50 & 1.46 & .233 \\
Instruction condition & 1,50 & $125.33 * * *$ & $<.001$ \\
Congruency & 1,50 & $426.58^{* * *}$ & $<.001$ \\
CS x Rule group & 1,50 & 1.02 & .317 \\
CS x Instruction condition & 1,50 & 1.44 & .235 \\
Rule group x Instruction condition & 1,50 & 0.08 & .785 \\
CS x Congruency & 1,50 & 1.42 & .2395 \\
Rule group x Congruency & 1,50 & 0.79 & .378 \\
Instruction condition x Congruency & 1,50 & 3.46 & .069 \\
CS x Rule group x Instruction condition & 1,50 & 0.65 & .424 \\
CS x Rule group x Congruency & 1,50 & 0.33 & .568 \\
CS x Instruction condition x Congruency & 1,50 & 0.46 & .500 \\
Rule group x Instruction condition x Congruency & 1,50 & 1.09 & .301 \\
CS x Rule group x Instruction condition x Congruency & 1,50 & 0.02 & .890 \\
\hline
\end{tabular}

Note. $\mathrm{CS}=$ conscientiousness, $d f=$ degrees of freedom; $F=$ F-value; $p=$ p-value

\subsubsection{Post-error slowing}

Mean PES values are illustrated in Figure 16. A linear mixed-effects model with between-group factors conscientiousness (continuous) and rule group (RV vs. RR), as well as repeated measures factor congruency (congruent vs. incongruent) was computed. The main effect of conscientiousness, $F(1,50)=0.15, p=.697$, indicating that conscientiousness does not influence PES. Other effects remained non-signifcant, $F \mathrm{~s}<1.63, p \mathrm{~s}>.207$, see Table 9 for detailed inferential statistics. 


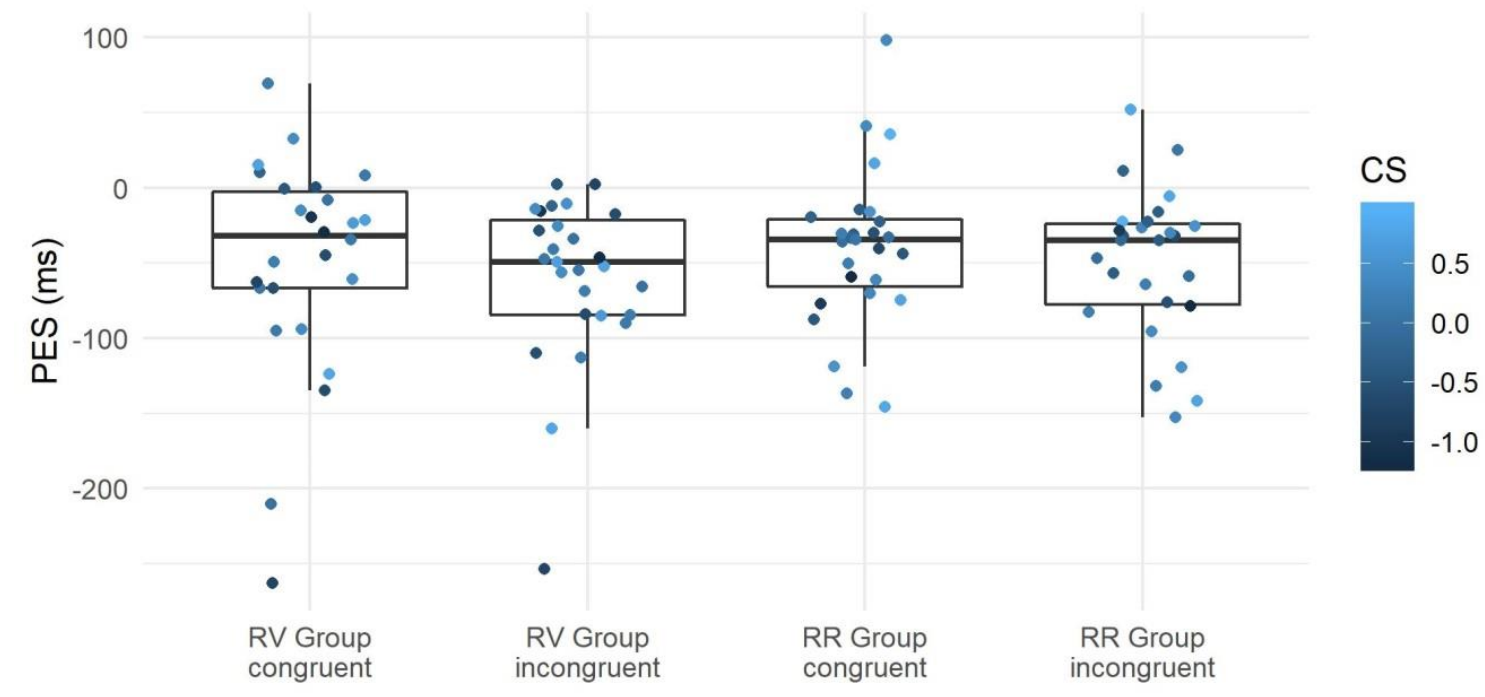

Figure 16. Mean PES in ms from standard instruction condition is displayed separately for each congruency condition (congruent, incongruent) separated by rule group, rule violation (RV), and rule reversal (RR). Scatter plots overlaying each boxplot display the PES as a function of conscientiousness (CS), the extent of which is represented by color intensity (high CS values are represented by low color intensity).

Table 9. Statistics of the linear mixed-effects regression model with PES as dependent variable.

\begin{tabular}{lccc}
\hline CS & $d f_{1}, d f_{2}$ & $F$ & $p$ \\
Rule group & 1,50 & 0.15 & 0.697 \\
Congruency & 1,50 & 0.57 & 0.454 \\
CS x Rule group & 1,50 & 1.27 & 0.265 \\
CS x Congruency & 1,50 & 0.06 & 0.810 \\
Rule group x Congruency & 1,50 & 1.63 & 0.207 \\
CS x Rule group x Congruency & 1,50 & 0.01 & 0.919 \\
\hline
\end{tabular}

Note. $\mathrm{CS}=$ conscientiousness, $d f=$ degrees of freedom; $F=$ F-value; $p=$ p-value. 


\subsubsection{ERPs}

For analysis of electrophysiological data, a series of linear mixed-effects regression analyses for the dependent variables ERN, Pe, CRN and Pc amplitude were conducted.

\subsubsection{ERN}

Due to the low error rate across the experiment, not every factor could be considered for the statistical analysis of the error-related potential. As criteria for considering factor levels or factors in statistical analysis, only levels of the factors rule group, instruction condition and congruency with at least 15 participants and only participants having at least 5 error trials were considered. After applying these criteria, only incongruent STD trials were left. The response-locked ERP averages of these trials are depicted in Figure 17. For this comparison 24 participants were excluded because of an insufficient number of error trials or the occurrence of artefacts in EEG data.

To analyze differences in ERN amplitude, a linear mixed-effects model regression analysis with the between-subject factor conscientiousness (continuous) and repeatedmeasures factor accuracy (correct vs. error) was performed. There was a highly significant main effect of accuracy, $F(1,33)=18.90, p<.001$, with participants having higher amplitudes after errors $(M=-8.98, S E=1.47)$ than after correct responses $(M=-2.05, S E=.76)$. The other effects were non-significant, $F \mathrm{~s}<0.01, p \mathrm{~s}>.917$.

\subsubsection{2 $\mathrm{Pe}$}

In order to analyze differences in Pe amplitude, a linear mixed-effects model regression analysis with the continuous factor conscientiousness and repeated-measures factor accuracy (correct vs. error) was performed. There was a highly significant main effect of accuracy, $F(1,33)=59.90, p<.001$, with participants having higher amplitudes after errors $(M=5.07 \mu \mathrm{V}, S E=1.13 \mu \mathrm{V})$ than after correct responses $(M=-9.54 \mu \mathrm{V}, S E=1.61 \mu \mathrm{V})$. Other effects were non-significant, $F \mathrm{~s}<0.40, p \mathrm{~s}>.531$. 


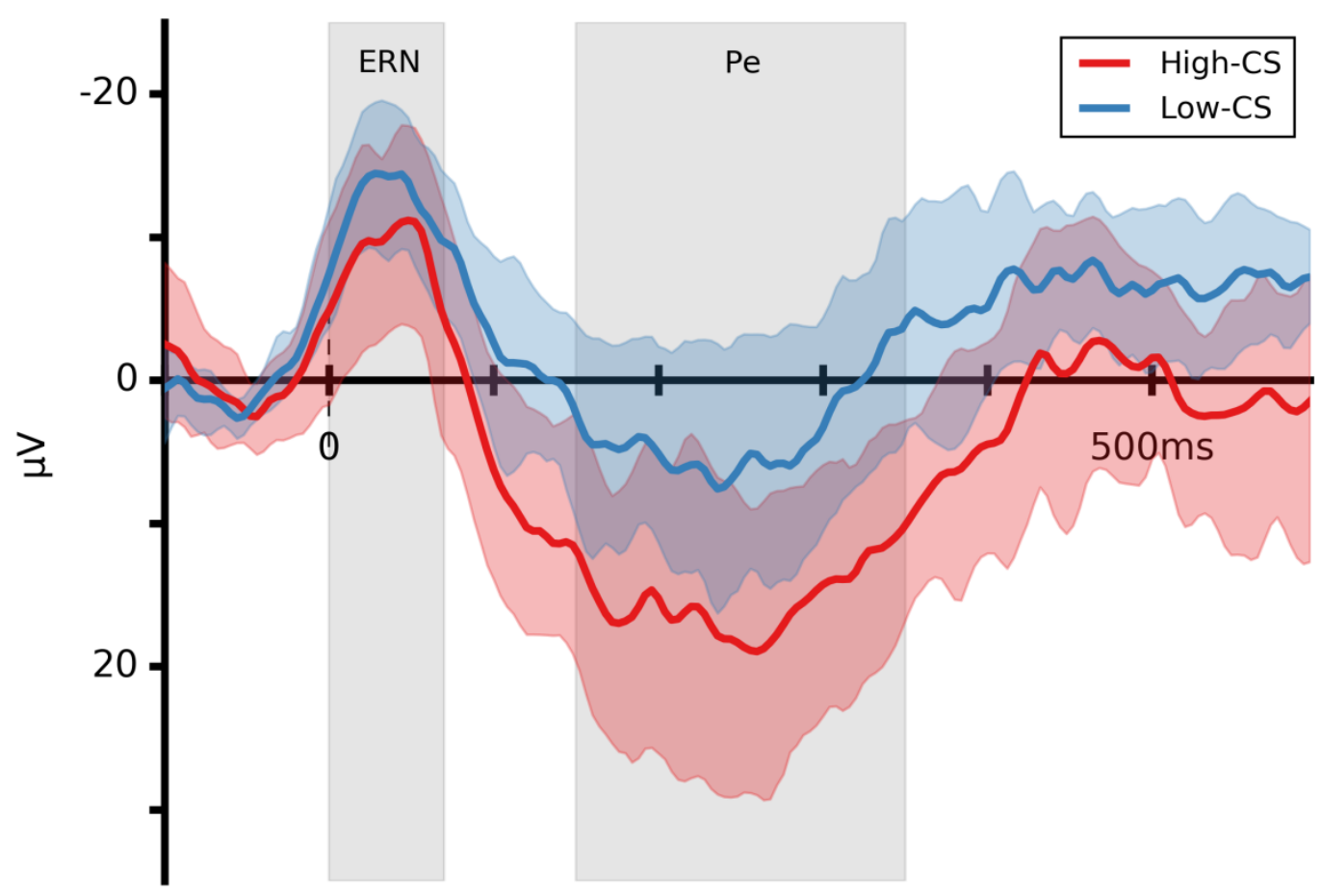

Figure 17. Response-locked ERP at electrode $\mathrm{FCz}$ after errors in incongruent standard trials separated by levels of conscientiousness (CS; low, high). Shaded areas indicate the $95 \%$ confidence interval of the mean ERPs.

\subsubsection{CRN}

The response-locked ERP averages of trials with correct responses separated by groups of conscientiousness (low or high) for the purpose of illustration are depicted in Figure 18. Topographical maps are depicted in Figure 19 and Figure 21. 

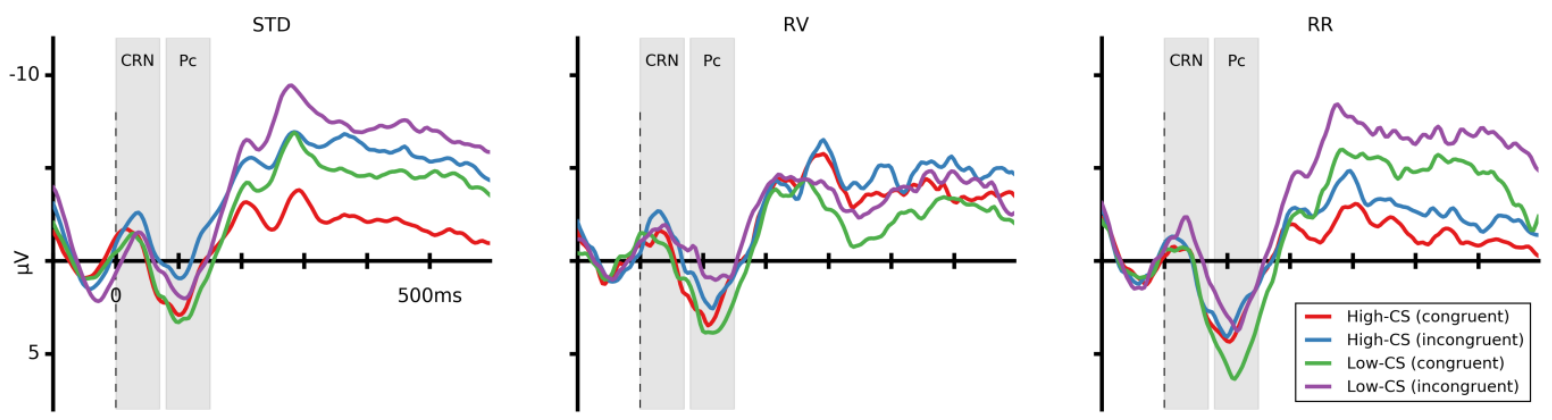

Figure 18. Response-locked ERP at electrode $\mathrm{FCz}$ following correct responses in standard (STD) instruction condition as well as alternative instruction conditions rule violation (RV) and rule reversal (RR) separated by levels of conscientiousness (CS; low, high) and congruency (congruent, incongruent). For the purpose of illustration low and high conscientiousness groups were formed according to the sample selection.

The response-locked mean amplitude in $0-70 \mathrm{~ms}$ post-response time interval measuring the CRN was compared utilizing a linear mixed-effects regression model with betweengroups factors conscientiousness (continuous) and rule group (RV vs. RR), as well as repeated measures factors instruction condition (STD vs. ALT) and congruency (congruent vs. incongruent). The main effect of instruction condition was significant, $F(1,50)=11.42$, $p=.001$. The analysis also revealed a significant main effect of congruency, $F(1,50)=10.92, p=.002$. A significant three-way interaction including the factors conscientiousness, instruction condition and congruency was revealed, $F(1,50)=7.09, p=.010$. This indicated that the CRN amplitude responded differently to the experimental manipulations depending on conscientiousness. For a detailed summary of the analysis results see Table 10. 


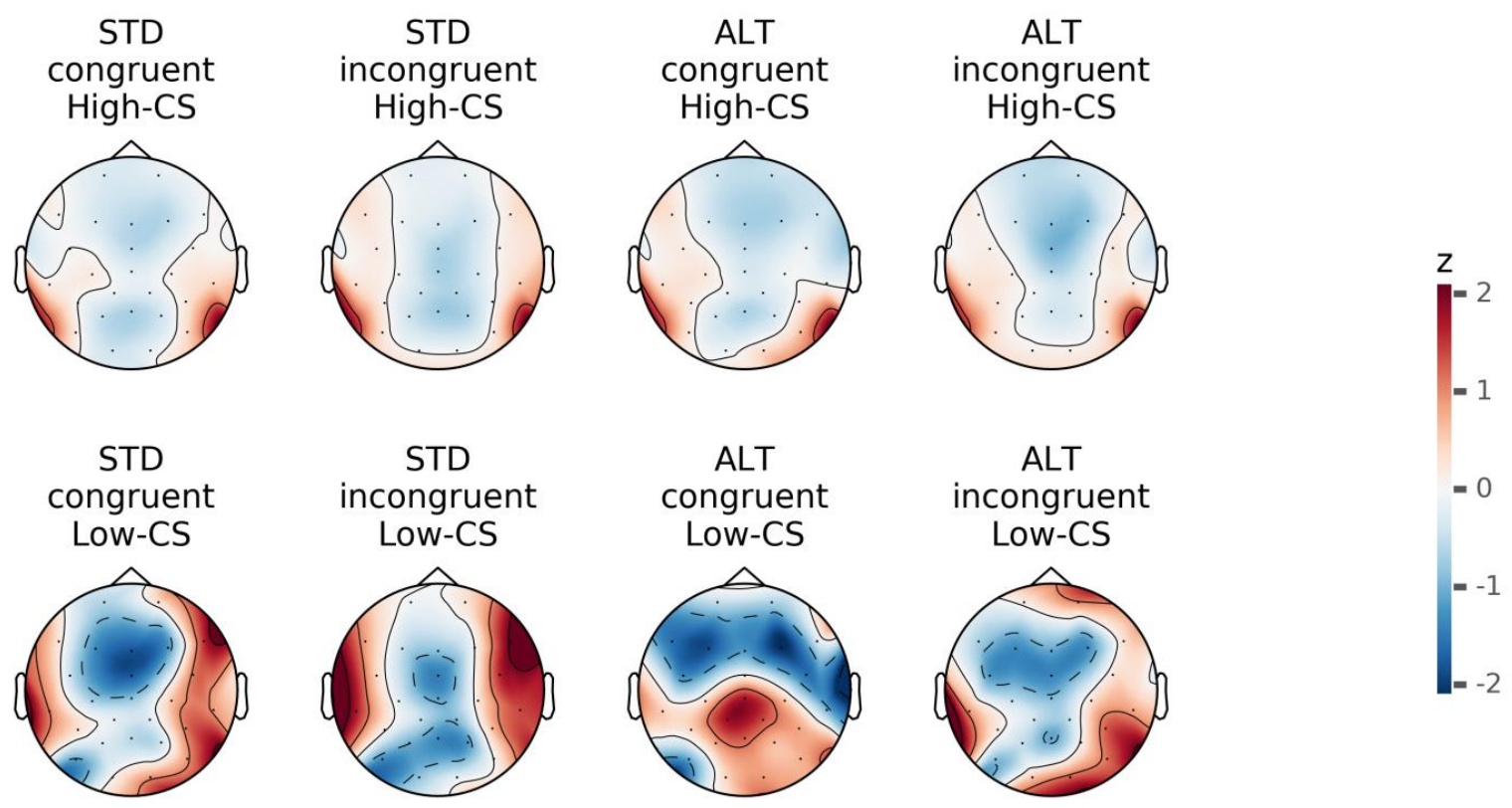

Figure 19. Z-transformed voltage distributions during response-locked ERP after correct responses in a time window of $10-40 \mathrm{~ms}$. The upper and lower heads show distributions of participants scoring high and low on conscientiousness (CS), respectively. The distributions of each group are displayed separately by column for the combinations of instruction conditions, i.e. standard (STD) and alternative (ALT), and congruency type, i.e. congruent and incongruent. Note: Rule violation and reversal instruction condition were taken together because there were no significant differences between both conditions. 
The impact of conflict on performance monitoring in conscientious individuals

Table 10. Statistics of the linear mixed-effects regression model with mean amplitude in $0-70 \mathrm{~ms}$ post-response time interval measuring the CRN in ERP after correct responses as dependent variable.

\begin{tabular}{lccc}
\hline & $d f_{1}, d f_{2}$ & $F$ & $p$ \\
\hline CS & 1,50 & 0.03 & .865 \\
Rule group & 1,50 & 1.74 & .193 \\
Instruction condition & 1,50 & $11.42^{* *}$ & .001 \\
Congruency & 1,50 & $10.92^{* *}$ & .002 \\
CS x Rule group & 1,50 & 0.06 & .812 \\
CS x Instruction condition & 1,50 & 1.17 & .285 \\
Rule group x Instruction condition & 1,50 & 0.22 & .064 \\
CS x Congruency & 1,50 & 0.45 & .504 \\
Rule group x Congruency & 1,50 & 0.53 & .472 \\
Instruction condition x Congruency & 1,50 & 1.16 & .286 \\
CS x Rule group x Instruction condition & 1,50 & 1.37 & .435 \\
CS x Rule group x Congruency & 1,50 & 0.32 & .573 \\
CS x Instruction condition x Congruency & 1,50 & $7.09 *$ & .010 \\
Rule group x Instruction condition x Congruency & 1,50 & 0.62 & .435 \\
CS x Rule group x Instruction condition x Congruency & 1,50 & 0.01 & .914 \\
\hline
\end{tabular}

Note. CS $=$ conscientiousness, $d f=$ degrees of freedom; $F=$ F-value; $p=$ p-value

To clarify the significant three-way interaction, a linear mixed-effects model analysis with repeated-measures factors instruction condition and congruency was conducted for both conscientiousness groups created prior to the experiment, separately. In the group with low values of conscientiousness, both the main effect of congruency, $F(1,23)=3.49$, $p=.075$, and the main effect of instruction condition approached significance, $F(1,23)=3.63, p=.069$. In the group with high values of conscientiousness, the analysis revealed a significant interaction of instruction condition and congruency, $F(1,29)=5.02$, $p=.033$ (for descriptive statistics see Figure 20). There was also a significant main effect of congruency, $F(1,29)=7.67, p=.010$ as well as a significant main effect of instruction condition, $F(1,29)=8.42, p=.007$. 

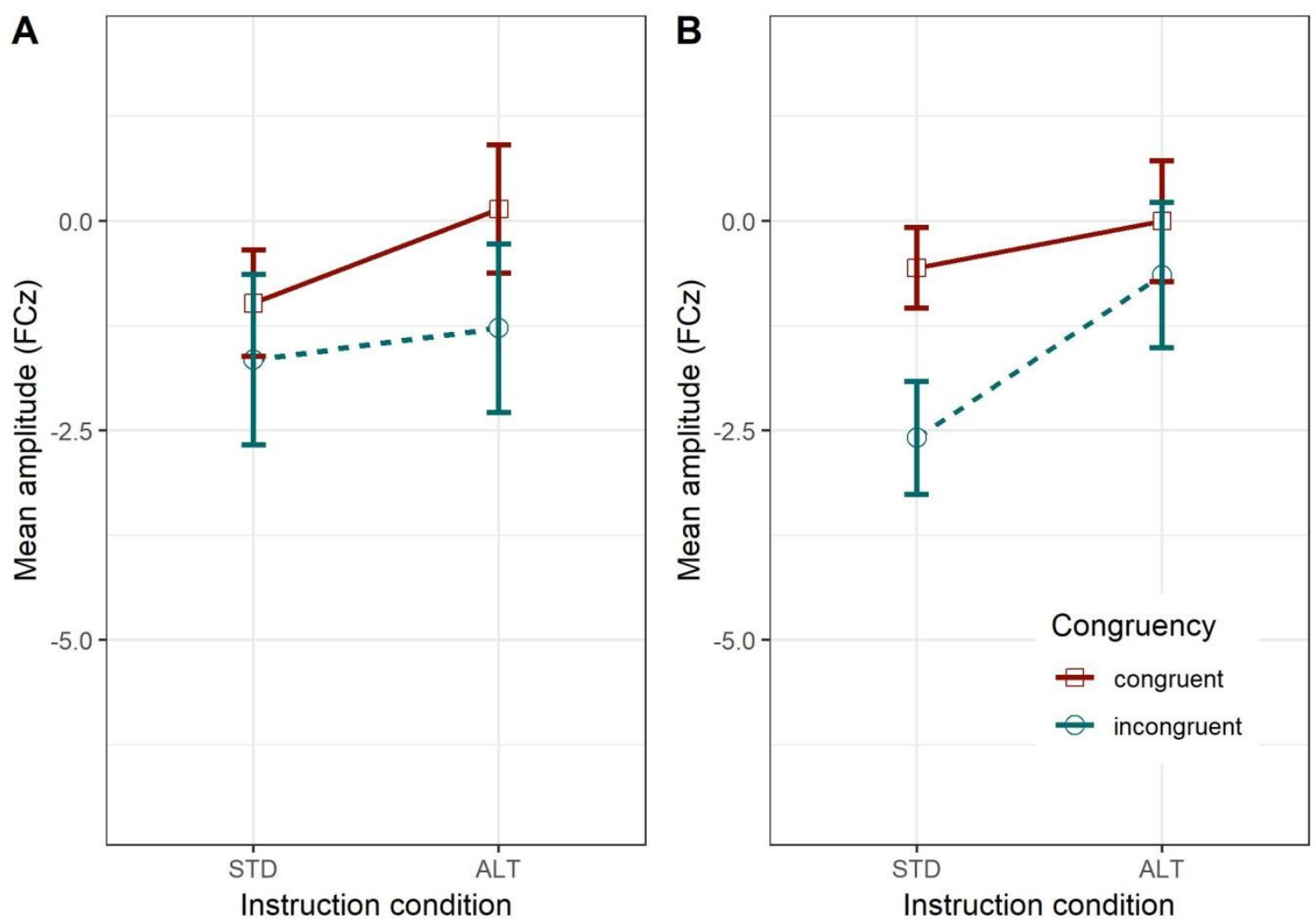

Figure 20. Mean amplitude during 0-70ms post-response time interval of the response-locked ERP after correct responses at electrode FCz. The mean amplitudes of individuals scoring low (A) and high (B) on conscientiousness (CS) scale are separately depicted for instruction conditions (STD, ALT) and congruency (congruent, incongruent). Error bars indicate standard error.

\subsubsection{Pc}

Another linear mixed-effects regression model with between-group factors conscientiousness (continuous) and rule group (RV vs. RR), as well as repeated measures factors instruction condition (STD vs. ALT) and congruency (congruent vs. incongruent) was calculated to analyze differences in the Pc. There was a significant three-way interaction effect including factors conscientiousness, instruction condition and congruency, $F(1,50)=9.87, p=.003$. There was also a highly significant main effect of instruction condition, $F(1,50)=33.57, p<.001$ as well as a highly significant main effect of congruency, $F(1,50)=12.88, p<.001$. Further inferential statistics are described in Table 11 . 
The impact of conflict on performance monitoring in conscientious individuals

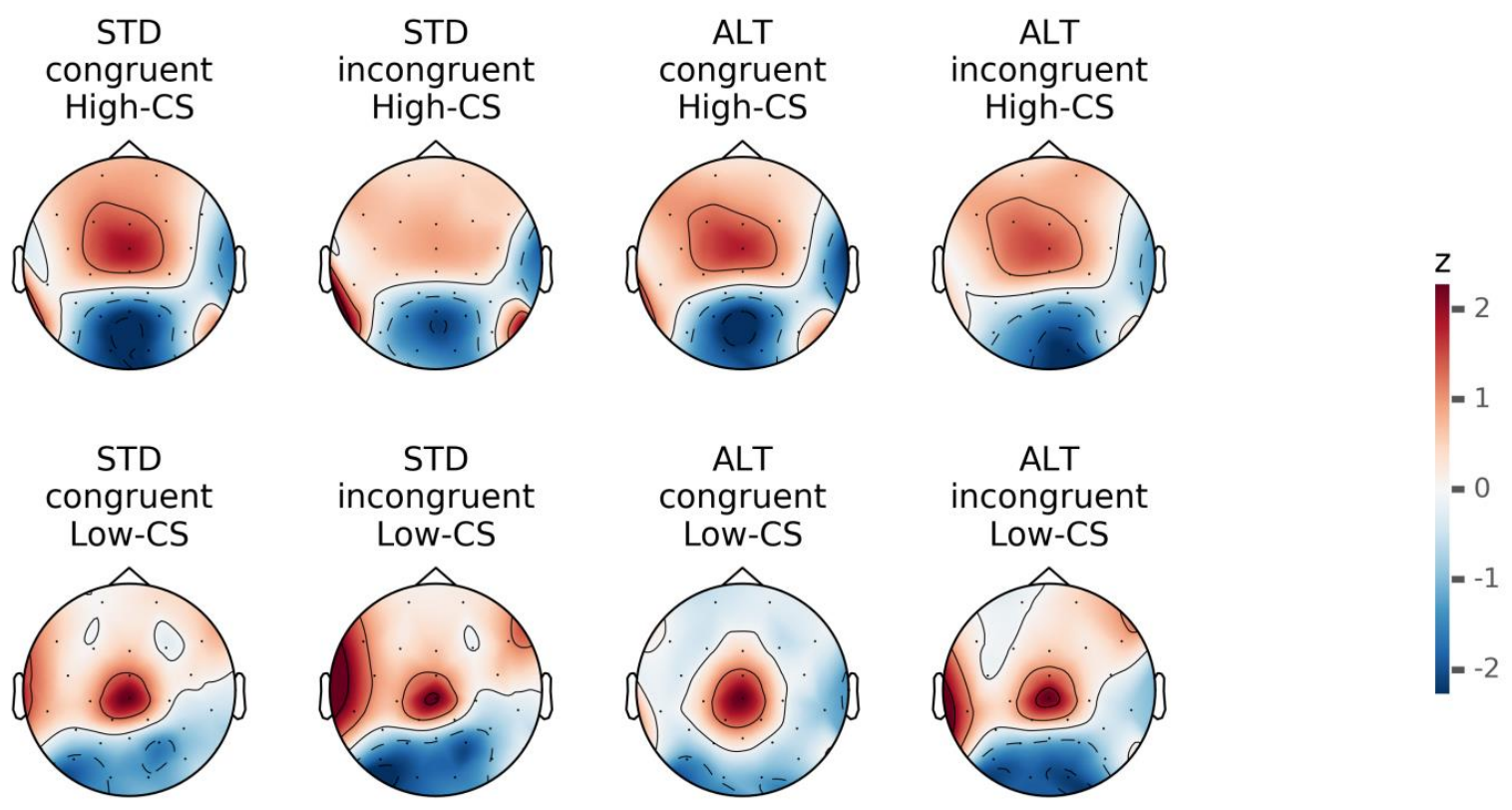

Figure 21. Z-transformed voltage distributions during response-locked ERP after correct responses in a time window of $90-110 \mathrm{~ms}$. The upper and lower heads show distributions of participants scoring high and low on conscientiousness (CS), respectively. The distributions of each group are displayed separately by column for the combinations of instruction conditions, i.e. standard (STD) and alternative (ALT), and congruency type, i.e. congruent and incongruent. Note: Rule violation and reversal instruction condition were taken together because there were no significant differences between both conditions. 
The impact of conflict on performance monitoring in conscientious individuals

Table 11. Statistics of the linear mixed-effects regression model mean amplitude in $80-150 \mathrm{~ms}$ postresponse time interval measuring the Pc in ERP after correct responses as dependent variable.

\begin{tabular}{lccc}
\hline & $d f_{1}, d f_{2}$ & $F$ & $p$ \\
\hline CS & 1,50 & 0.19 & .661 \\
Rule group & 1,50 & 0.75 & .391 \\
Instruction condition & 1,50 & $33.57^{* * *}$ & $<.001$ \\
Congruency & 1,50 & $12.88^{* * *}$ & $<.001$ \\
CS x Rule group & 1,50 & 0.10 & .757 \\
CS x Instruction condition & 1,50 & 0.36 & .553 \\
Rule group x Instruction condition & 1,50 & 0.43 & .517 \\
CS x Congruency & 1,50 & 1.96 & .168 \\
Rule group x Congruency & 1,50 & 0.64 & .427 \\
Instruction condition x Congruency & 1,50 & 1.09 & .301 \\
CS x Rule group x Instruction condition & 1,50 & 0.18 & .675 \\
CS x Rule group x Congruency & 1,50 & 0.44 & .509 \\
CS x Instruction condition x Congruency & 1,50 & $9.87^{* *}$ & .003 \\
Rule group x Instruction condition x Congruency & 1,50 & 0.39 & .536 \\
CS x Rule group x Instruction condition x Congruency & 1,50 & 0.97 & .329 \\
\hline
\end{tabular}

Note. $\mathrm{CS}=$ conscientiousness, $d f=$ degrees of freedom; $F=\mathrm{F}$-value; $p=$ p-value

To elucidate the significant three-way interaction, a linear mixed-effects model analysis with repeated-measures factors instruction condition and congruency was conducted for both conscientiousness groups created prior to the experiment, separately. The analysis for the group with low values of conscientiousness revealed a significant main effect of congruency, $F(1,23)=8.44, p=.008$, with participants having higher amplitudes during trials with congruent flanker stimuli $(M=1.85, S E=.73)$ than during trials with incongruent flanker stimuli $(M=.24, S E=.72)$. There was also a significant main effect of instruction condition, $F(1,23)=5.48, p=.028$, with participants showing higher amplitudes in ALT instruction condition $(M=2.20, S E=.78)$ than in STD instruction condition $(M=-.11$, $S E=.63)$. In the group with high values of conscientiousness, the analysis revealed a highly significant interaction of instruction condition and congruency, $F(1,29)=14.36$, $p<.001$ (for descriptive statistics see Figure 22) and in accordance with the results for the low conscientiousness group a significant main effect of congruency, $F(1,29)=13.03$, 
The impact of conflict on performance monitoring in conscientious individuals

$p=.001$, as well as a highly significant main effect of instruction condition, $F(1,29)=18.50, p<.001$.
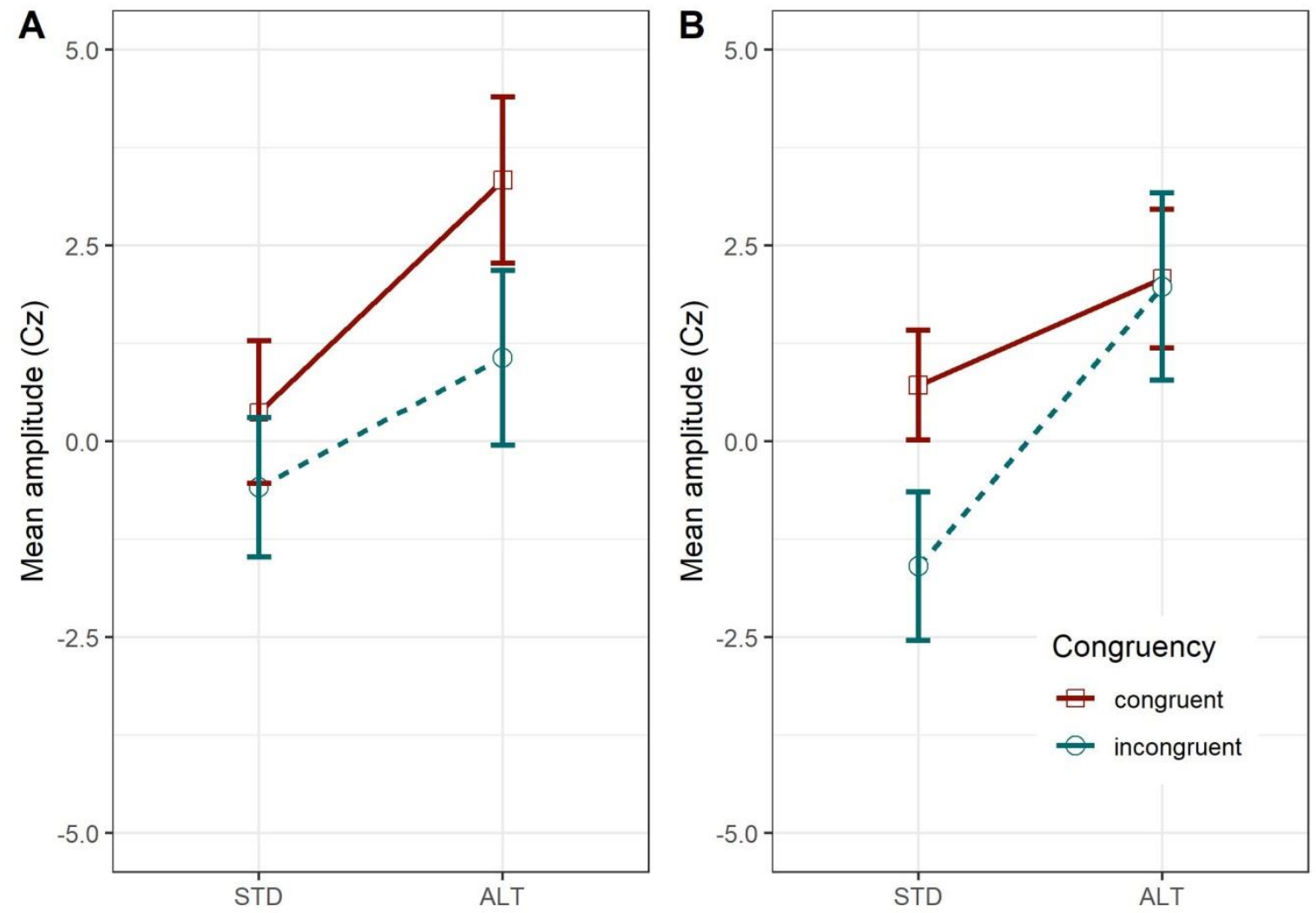

Figure 22. Mean amplitude during $80-150 \mathrm{~ms}$ post-response time interval of the response-locked ERP after correct responses at electrode $\mathrm{Cz}$. The mean amplitudes of individuals scoring low (A) and high (B) on conscientiousness (CS) scale are separately depicted for instruction conditions (STD, ALT) and congruency (congruent, incongruent). Error bars indicate standard error. 


\subsection{Discussion}

Experiment 2 aimed at solving methodological problems of Experiment 1 and to shed more light on the relationship of conscientiousness with ERP correlates of the performance monitoring system. Conscientiousness groups were formed following a pre-assessment of conscientiousness in participants and by only taking extreme values for low and high conscientiousness. Both conscientiousness groups were further split into a RV and a RR group. In this way, rule violations and rule reversals were tested in a between-group instead of a within-subject design in order to prevent internal reframing of instructions within subjects which was observed in Experiment 1. The task of the experiment consisted of an adopted Eriksen Flanker Task (Eriksen \& Eriksen, 1974). During a frequent condition (i.e. the STD condition) participants were instructed to respond to triangles pointing to the left or right by pressing the left or right button, respectively. In an infrequent condition (i.e. the ALT condition), participants from RV group were asked to violate the rules and subjects from RR group were instructed to reverse the rules.

The results showed an effect of congruency on RT and error rate consisting of faster responses and fewer errors during congruent compared to incongruent flanker trials. There was also a congruency effect on electrophysiological measures consisting of ERPs with larger CRN amplitudes after correct responses during incongruent flanker trials. The results partially replicated the findings from Experiment 1. First, there was an effect of the instruction condition on RT and error rate. Subjects responded faster and made fewer errors in the STD condition which was also the frequent condition in the present experiment. The instruction condition also affected the response-locked ERP, particularly the amplitude of the Pc which was generally higher in ALT condition. Second, the response-locked ERP at frontocentral electrodes after correct responses varied depending on conscientiousness, instruction condition and congruency, while there was no difference in RT and error rates between participants scoring low and high on the conscientiousness scale. This particular pattern showed that the congruency effect (consisting of a pronounced CRN amplitude after responses during incongruent trials mentioned before) was even stronger in conscientious individuals during STD trials. Furthermore, less conscientious participants showed a 
The impact of conflict on performance monitoring in conscientious individuals

distinction in Pc magnitude also depending on congruency and instruction condition with higher Pc amplitudes during incongruent STD trials.

Unfortunately, as in analysis of Experiment 1, the results concerning ERN amplitude could not be analyzed due to the small number of error trials that were left for ERP analysis except for incongruent STD trials. There was no difference between ERN amplitudes in individuals with low and high values of conscientiousness for incongruent STD trials.

As in Experiment 1, findings concerning ERN should be interpreted with caution. The number of errors generated overall was relatively small and even lower than in Experiment $1, M=5.07 \%, S E=.34$. As a result only few trials were left for analysis of variations in ERN amplitude after artefact rejection. I only included participants with at least 5 error trials in my analyses. However, it is recommended to consider at least 15 trials for withinsubject comparisons (Fischer et al., 2017). Future research may aim at further improving the experimental design to force participants to commit more errors.

\subsubsection{Task engagement in conscientious individuals}

Incongruent flanker stimuli elicited larger CRN amplitudes than congruent stimuli. This congruency effect on the CRN has been reported before (Allain et al., 2004; Bartholow et al., 2005; Botvinick et al., 1999; Carter et al., 1998; Simons, 2010). Most importantly, the present experiment also revealed similar differences in CRN and Pc amplitude as in Experiment 1. In contrast, however, these differences did not appear to be true for all conditions. The obtained data from the present experiment showed higher CRN amplitudes in conscientious individuals during incongruent STD trials. In line with other studies (Hajcak \& Simons, 2002; Kałamała et al., 2018; Simons, 2010), results from Experiment 1 suggest that higher CRN amplitude is related to an increased task engagement or an accuracy-focused strategy (cf. section 2.4.1). According to this perspective, the higher CRN amplitudes in conscientious individuals during incongruent STD trials could be explained by differences in the task engagement with a specific focus on this trial type. Incongruent stimuli are characterized by higher response conflict than congruent ones. Thus, the complexity of the task and the difficulty of incongruent stimuli might cause conscien- 
tious individuals to specifically focus on this trial type and pay relatively less attention to other trial types. However, there are some reasons not to believe that this explanation fully covers the obtained data of the present experiment. When conscientious individuals pursue an accuracy-focused strategy, their task performance would show lower error rates. The present experiment however only showed a tendency towards lower error rates in conscientious participants, while the RTs remained the same between both conscientiousness groups. A similar behavioral pattern has also been observed in Experiment 1. If the assumption holds that the accuracy-focused strategy varies depending on conscientiousness, congruency and instruction, some questions remain unanswered and have to be addressed in future research. For instance, what factors lead to these observed task-related strategy adaptations in conscientious individuals? Why do they focus on specific trial types?

In line with results of Experiment 1 showing a more pronounced Pc during infrequent tasks, the ALT condition of the present experiment also elicited generally higher Pc amplitudes in the participants. An increased Pc amplitude was also observed in an infrequent task by Hajcak and colleagues (2004). It could reflect a process that controls how to respond next based on an expectation of the upcoming trial type. However, this interpretation is rather speculative and needs further investigation in future experimental settings possibly involving trial types with different frequency levels. In past studies the Pc amplitude was also found to be larger in trials preceding errors (Allain et al., 2004; Hajcak, Nieuwenhuis et al., 2005) and it was interpreted as disengagement of the ACC from the performance monitoring process (Ridderinkhof et al., 2003). An increased Pc amplitude was also found to be associated with negative affect (Hajcak et al., 2004).

The association of conscientiousness with the Pc amplitude depended on the same conditions as the CRN amplitude. The Pc magnitude varied depending on instruction condition and congruency. Participants scoring low on the conscientiousness scale showed higher Pc amplitudes when confronted with incongruent stimuli combined with the instruction to respond according to the rules. 


\subsubsection{Effects of rule violations}

The present experiment showed a clear behavioral effect of inhibiting the rulecompliant S-R association when reversing or violating the rules. This cognitive effort was expressed by higher error rates and higher RTs. There was also an effect of the instruction condition on the Pc with higher amplitudes in ALT condition. However, neither behavioral nor ERP data from the present experiment indicated a difference between reversing and violating a rule, indicating the same quality of cognitive conflict in both conditions. This pattern is in line with results from Experiment 1. The assumption that a behavioral and electrophysiological signature of rule violations might be observed in a between-group design did not hold. In sum, these results are not in line with other research on rule violation behavior (Pfister, 2013; Wirth et al., 2016) showing differences in rule violation and rule-based behavior in a between-group design. It is difficult to identify the reasons for these diverging results. A speculative reason might be that instructions did not generate an ecologically valid feeling of violating a rule in the participants as they were legitimized by the instruction to do so in the present experiment.

\subsubsection{Conclusion}

To summarize, individual differences in CRN and Pc amplitude that are associated with conscientiousness are pointing in the same direction as in Experiment 1. However, differences between observations in both experiments might have arisen from the different task design. It is characterized by enhanced complexity due to the additional factor of flanker congruency as interactions of conscientiousness and congruency have indicated. Unfortunately, lower or equal error rates in the present experiment showed that the goal to force participants committing more errors was not achieved. Nevertheless, the present study delivers further support for the perspective that CRN is linked with task engagement or accuracy-focused performance strategy. Similarly, the association of individual differences in Pc amplitude with conscientiousness varied depending on the same conditions as the CRN amplitude which strengthens the assumption that the Pc reflects task disengagement. 


\section{Task-related ERPs as biomarkers for classification of conscientiousness}

\subsection{Introduction}

There is sufficient evidence to assume that individual differences in ERPs are associated with psychopathology and personality traits. A comprehensive overview was already delivered in a previous part of this thesis (see e. g. section 1.1.6). There are studies that show associations of individual differences in ERPs and psychopathology, e. g. including depression (e.g. Liu et al., 2014), schizophrenia (e.g. Horan, Foti, Hajcak, Wynn, \& Green, 2012), generalized anxiety disorder (e.g. Weinberg, Olvet, \& Hajcak, 2010), and obsessive compulsive disorder (e.g. Gehring et al., 2000). While research concerning the relationship of individual differences in ERPs with psychopathological characteristics is quite comprehensive, there are only few studies that observed ERPs to identify associations of neural responses and personality traits (e.g. Pailing \& Segalowitz, 2004).

Analyzing individual differences in ERPs is quite difficult and there are some methodological challenges as already brought up by Stahl and colleagues (2012). A fundamental problem is the low signal-to-noise ratio of ERP recordings. A standard procedure is to average the response over a large number of trials (Luck, 2014) with the intention to filter out noise unrelated to stimulus presentation or response processing. The result should be an ERP waveform that is only related to the stimulus or response processing. The analysis of ERPs commonly involves univariate group comparisons, e.g. comparisons of mean ERP amplitudes between an experimental group and a control group, or comparing participants ERP characteristics between two or more experimental conditions. Differences between groups are usually assessed for more than one amplitude and latency at several electrode locations (either taking single electrodes or averaging a group of electrodes). This proce- 
dure consists of multiple testing and leads to either an increase of family-wise type I error or a decrease of power when methods such as Bonferroni correction are used for adjusting the alpha error level (Stahl et al., 2012).

The existence of individual differences in ERPs that are associated with psychopathology or personality traits enables the possibility to measure ERPs for prognostic and diagnostic purposes. Practical advantages such as low costs and ease of use make EEG feasible and attractive for measuring brain functions and diagnostic biomarkers (McLoughlin, Makeig, \& Tsuang, 2014). In recent decade, machine learning methods such as linear discriminant analysis (LDA) and support vector machines have become popular for the classification of single trial or continuous EEG signals, e.g. in brain-computer interface studies (Blankertz, Lemm, Treder, Haufe, \& Müller, 2011; Lotte, Congedo, Lécuyer, Lamarche, \& Arnaldi, 2007). Recent approaches showed that it is also possible to identify individuals with psychopathological characteristics using machine learning methods. These approaches cover a variety of individual characteristics. Discrimination between infants with high and low risk of a later diagnosis of autism was achieved by use of support vector machines and regularized LDA (Stahl et al., 2012). Individuals with attention deficit hyperactivity disorder were successfully identified by means of support vector machines classifying independent ERP components (Mueller et al., 2011; Mueller, Candrian, Kropotov, Ponomarev, \& Baschera, 2010; Tenev et al., 2014). Depression patients were tried to discriminate from controls using logistic regression and LDA among others (Hosseinifard, Moradi, \& Rostami, 2013). Individual differences that indicate the onset of psychopathology were identified by classification of reward-related ERPs (Ethridge \& Weinberg, 2018). The ERN might also be a suitable candidate for a biomarker that could possibly be used for diagnostic instruments due to its relationship to individual differences in psychopathology. Larger differences between the ERN and CRN (i.e. $\triangle \mathrm{ERN}$ which is used to isolate neural activity specific to error processing) are related to clinically diagnosed general anxiety disorder (Meyer, Lerner, Reyes, Laird, \& Hajcak, 2017) and has been used to identify individuals with this disorder.

The existing approaches seem to be quite promising. However, there is need for investigations of psychometric properties of ERPs before they can reliably be used as bi- 
omarkers for diagnostic and prognostic purposes (Baldwin, Larson, \& Clayson, 2015; Ethridge \& Weinberg, 2018; Foti, Kotov, \& Hajcak, 2013; Hajcak, Meyer, \& Kotov, 2017; Kompatsiari, Candrian, \& Mueller, 2016; Meyer et al., 2017).

No studies so far have used ERPs trying to identify differences in conscientiousness or other personality traits. However, there is evidence that conscientiousness might be associated with task-related ERPs (derived from Experiment 1 and 2; Hill et al., 2016; Pailing \& Segalowitz, 2004).

The aim of the present study is to analyze the ERP data that was acquired in Experiments 1 and 2 with recent analytical machine learning methods that are used for group discrimination, namely LDA with shrinkage of the covariance matrix (that is the regularized form of LDA) and LR. Both methods are well suited to analyze differences between groups and allow for data analysis with a large number of variables in relation to the sample size while they avoid multiple testing problems of standard analysis procedures. The predictive power of the resulting models is evaluated by cross-validation methods. The present study primarily aims at finding further evidence for individual differences in the response-locked ERP that are associated with conscientiousness. The study secondarily aims at exploring potential applications of machine learning methods for detecting an electrophysiological signature of conscientiousness.

In the present chapter, I propose a procedure that can be used to classify dispositional characteristics using ERP data containing biomarkers, recorded during tasks like they are used in Experiment 1 and 2. I also describe and evaluate a concrete implementation of the proposed procedure that aims at the classification of conscientiousness. The procedure is based on building separability indices that consist of Pearson r-values calculated from voltage and conscientiousness values for each combination of a channel and scan point of an EEG epoch. They help to determine time windows containing spatio-temporal features with highest discriminative value. The voltage values within the selected time windows are averaged for each epoch of a subject and are used for classification learning. Finally, the procedure is evaluated by means of a 6 -fold cross validation and calculating performance metrics accuracy and receiver operating characteristic (ROC). 


\subsection{Approach for classification of conscientious- ness using task-related ERPs}

This chapter focuses on the procedure of my approach for classification of conscientiousness.

\subsubsection{Feature extraction}

Principally, it is possible to take the raw ERP record and feed supervised classification methods with it. However, it is helpful to do data pre-processing and reduction to extract a set of data features beforehand. After removing trials with artefacts, there are possible techniques that aim to reduce the signal-to-noise ratio including temporal and spatial filters like bandpass, notch, or Laplace filters (Luck, 2014). A popular method for artefact correction is blind component separation using independent component analysis (ICA; Joyce et al., 2004; Kierkels et al., 2006) is. ICA can also be used to extract features for ERP classification. ICA decomposes sensory single trial data which is derived from several electrodes and its result is a linear combination of temporally independent components in relation to modality and spatial location of signals. The components obtained in this way are assumed to reflect activity of different brain areas during a trial. One issue with ICA methods is that they often need larger sample sizes than are available.

Another technique is building separability indices and is based on visual inspection. To date, it was successfully applied in singe-trial analysis (Blankertz et al., 2011). It helps to get an overview of where the discriminative information lies and to control selecting features with theoretical foundations in mind. In its essence, it consists of a visualized matrix of separability measures for each pair of time point and channel to contrast ERP records of experimental conditions or groups. Ideally, segmented data is used as basis for the calculation. To calculate the separability information of two experimental conditions or groups, different measures can be used such as point-biserial correlation coefficient, Student's $t$ statistic (Student, 1908), area under the ROC curve (Green \& Swets, 1966) or rank-biserial correlation coefficient (Cureton, 1956). The selection of a suitable measure depends on the properties of the underlying dataset. In contrast to point-biserial correlation coefficient and 
Student's $t$-statistic, area under the ROC curve and rank-biserial correlation coefficient do not rely on the assumption that features are normally distributed for both classes. The separability information can then be displayed as a color coded matrix as it is exemplary shown in Figure 23.

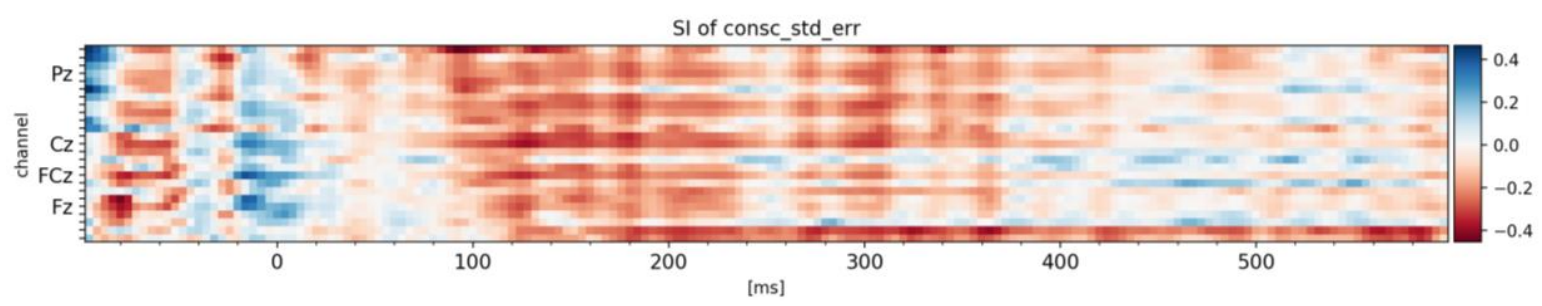

Figure 23. Exemplary visualization of spatio-temporal features that consist of a matrix with signed- $r^{2}$ values of low- minus high-CS averaged ERPs. The matrix is displayed as color coded matrix.

For the classification described in this work, signed- $r^{2}$-values of the point-biserial correlation coefficient are used that is defined as

$$
r(x):=\frac{\sqrt{N_{1} * N_{2}}}{N_{1}+N_{2}} \frac{\operatorname{mean}\left(x_{i} \mid y_{i}=1\right)-\operatorname{mean}\left(x_{i} \mid y_{i}=2\right)}{\operatorname{std}\left(x_{i}\right)}
$$

Based on the matrix of $r^{2}$ values that is displayed in Figure 23, it is possible to identify a set of time intervals that are suitable for classification. The average across time is calculated within each interval, hence it should be considered to have a spatial pattern that is as homogenous as possible during selection of intervals.

\subsubsection{Linear classification techniques}

Linear classifiers consist of algorithms that use linear functions to distinguish between classes. They probably are the most popular classifiers used in BCI applications. This section focuses on describing Linear Discriminant Analysis (LDA) and Logistic Regression (LR) which are statistical methods that are used to analyze categorical outcome variables,.

\subsubsection{Linear discriminant analysis}

LDA is similar to Fisher Discriminant Analysis and Least Squares Regression. It is one of the most commonly used techniques for discriminating between groups of subjects or 
trials within one subject in ERP scenarios. Unlike other methods used for classification, LDA allows for assessing the contribution of each variable to discriminate between groups which helps to describe differences between groups. LDA aims to find a linear combination of variables separating two or more classes of objects or events. Regarding a two-class problem, LDA discriminates using a linear discriminant function that is placed between the centroids of the two classes. Given the instance is represented as a vector, the class of the instance is determined by the side of the function on which the vector is located. For calculation of the linear discriminant function, the correlation between feature variables is taken into account. The linear discriminant function can be expressed as

$$
w_{0}+w^{T} x=0
$$

where $x$ is a feature vector and the projection vector is defined as

$$
w=\hat{\Sigma}_{c}^{-1}\left(\hat{\mu}_{2}-\hat{\mu}_{1}\right)
$$

where $\hat{\mu}_{i}$ is the estimated mean of class $i$ and $\hat{\Sigma}_{c}=\frac{1}{2}\left(\widehat{\Sigma}_{1}+\hat{\Sigma}_{2}\right)$ the estimated common covariance matrix. To estimate the covariance matrix, the empirical covariance matrix is commonly used.

When $x_{1}, \ldots, x_{n}$ are $n$ feature vectors and when

$$
\hat{\mu}=\frac{1}{n} \sum_{i=1}^{n} x_{i}
$$

is the unbiased estimator of the mean. It is possible to estimate the covariance matrix by the empirical covariance matrix:

$$
\hat{\Sigma}=\frac{1}{n-1} \sum_{i=1}^{n}\left(x_{i}-\hat{\mu}\right)\left(x_{i}-\hat{\mu}\right)^{T}
$$

As can be shown, LDA is an optimal classifier insofar as it minimizes the risk of misclassification of new samples drawn from the identical distribution, when known normal distributions have the same covariance matrix. Even when it is not optimal, one might ac- 
cept a loss in performance to take the advantage of its simplicity (Duda, Hart, \& Stork, 2001).

To yield an optimal classification, LDA makes assumptions regarding distribution of the features which are listed below:

1. Features of each class are normally distributed. Generally, ERP data satisfy this condition well (Blankertz et al., 2011). However, LDA is still robust to deviations from the normality assumption.

2. Normal distributions of all classes share an equal covariance matrix. This requirement is necessary to ensure that the data is linear separable. This assumption is presumably satisfied for most ERP data sets (Blankertz et al., 2011). Here again, LDA is quite robust and yields sufficient results, even when this assumption is not fulfilled.

3. True class distributions are known. However, it should be mentioned that this assumption is never fulfilled in any real application and it is therefore necessary to rely on means and covariance matrices estimated from the training data. Even if the assumptions (1) and (2) are sufficiently fulfilled, the optimality criterion might not hold at all due to error in those estimates. This problem might specifically occur when having a small number of training samples compared to the dimensionality of features which is sometimes referred as the curse-ofdimensionality (Lotte et al., 2007).

In cases of data with high dimensionality but only a few data points given, it is recommended to use shrinkage as regularization method. The number of unknown parameters is a quadratic function of the number of dimensions. The higher the dimensionality of features with a concurrent small number of training samples, the empirical covariance matrix (see Equation 5; i.e. the standard estimator) becomes more imprecise. This is a common problem in classification of ERPs because in most cases there is a high number of dimensions due to different time intervals in the spatio-temporal domain and low number of training samples due to problems of feasibility of experimental designs with high numbers of trials or participants. Shrinkage is an appropriate solution to compensate a bias caused 
by an imprecise estimation of the covariance matrix (Friedman, 1989). In order to counteract the imprecise estimation, $\hat{\Sigma}$ can be replaced by

$$
\tilde{\Sigma}(\gamma):=(1-\gamma) \hat{\Sigma}+\gamma v I
$$

where $I$ being the identity matrix and $v$ the average eigenvalue trace $(\hat{\Sigma}) / d$ of $\widehat{\Sigma}$ with $d$ being the dimensionality of the feature space. $\gamma \epsilon[0,1]$ is a tuning parameter. A modification of the covariance matrix in this way results in a regularized LDA, covarianceregularized LDA or LDA with shrinkage.

\subsubsection{Logistic regression}

An alternative to LDA is logistic regression (LR). Despite its name, LR is a linear classification model rather than a regression. It is also known as logit regression, maximumentropy classification or log-linear classifier. The aim of LR is finding the best fitting and most parsimonious model for determining the class of an object or event (dependent variable) as a function of its features (independent variables). A model based on LR utilizes a logistic function to calculate probabilities that describe the possible outcomes.

The LR model can be expressed as

$$
P(Y=1)=\frac{e^{w^{T} x}}{1+e^{w^{T} x}}
$$

where $x$ is a feature vector.

The maximum likelihood method is used to estimate the coefficients.

The method is quite robust, flexible and easy to use (Pohar, 2004). A main advantage of LR is that it does not make any assumptions regarding the distribution of the feature variables which is a potential drawback of LDA. However, there are few practical differences between LDA and LR (Michie, Spiegelhalter, \& Taylor, 1994). There is also a regularized LR for high-dimensional data (Harrel, 2015) similar to regularized LDA. 


\subsection{Validation of classification models}

An important aim of this study is the evaluation of different classification methods with regard to how well the resulting models are able to generalize to unseen data. To gain insight in which method is most suitable for classification of conscientiousness, comparison of classification methods is also helpful. Several methods can be applied to quantify the quality of predictions. Supervised learning techniques are inductive, which means that they acquire the shape of their function from data. Therefore, it is necessary to assess the quality of predictions of a classification model by using validation procedures. Testing a classification model on the same data that was used for learning the parameters of this model is not a proper method for its evaluation because it overestimates the true classification rate. In this case the result would be a perfect score failing to give useful predictions on unseen examples. In case of parameter optimization this situation would even lead to overfitting. In case of estimating the generalization error of a classification model, this procedure would lead to a bad estimate.

To avoid this situation, it is common to use external methods which test classification performance with a sample that was not used by the classification learning algorithm. In case of large sample sizes, the data set can be randomly divided into training and test samples. By applying this hold-out method, the classification model will be constructed on basis of the training sample, while the classification performance will be assessed using the test sample. If the classification performance is measured by accuracy, true performance is estimated by the percentage of test sample cases which are classified correctly.

In many cases the sample size is not large enough for splitting the sample in this way. A more suitable approach for small data sets is $k$-fold cross-validation (Goutte, 1997; Kohavi, 1995; Rodríguez, Pérez, \& Lozano, 2010). For this procedure, the whole data set is randomly split into $k$ smaller sets (folds). The classification model is derived from the training data set which comprises $k-1$ of the folds. The resulting model is then validated on the remaining part of the data. That includes computing a performance measure such as accuracy. The procedure is repeated until every fold is used as test set once. The estimated performance of the classification method is calculated by averaging the performance 
measure over the $k$ test sets. To improve the stability of the estimated performance, the $k$ fold cross-validations can be repeated several times by taking new random subsamples and then averaging the results.

During $k$-fold cross-validation the whole data set is divided into $k$ sets of equal sizes if it is possible. A variation is stratified $k$-fold cross-validation where training and test sets contain the same proportion of cases of each target class as the complete sample set. A special case of $k$-fold cross-validation is the leave-one-out method where each case is used as single test set while the remaining $n-1$ cases are used for deriving the classification model. The procedure is computationally more expensive than $k$-fold cross-validation when $n>k$, and there is evidence from simulation studies that this procedure can be unreliable and possibly underestimates the true predictive error. 5-fold to 10-fold crossvalidation has been shown to work better than leave-one-out (Breiman \& Spector, 1992; Kohavi, 1995) and is well suited for small sample sets (Martens \& Dardenne, 1998).

Another suitable method for evaluating classification methods is bootstrapping. Instead of taking subsets of the complete sample set as it is done during cross-validation, bootstrapping means taking subsamples of the full sample set repeatedly to assess the performance of a learned model. A subsample refers to a random sample with replacement from the complete sample set (Efron \& Tibshirani, 1993). Bootstrapping can be described as a technique to approximate the sampling distribution. How many subsamples are required depends on the research goal, it might be anywhere from 50 to 2000. Bootstrapping seems to be more suitable than cross-validation in many cases (Efron, 1983).

\subsubsection{Performance scoring}

The aim of assessing performance of a learned model is estimating the generalization error, or how well the model generalizes to unseen data. For this purpose, different performance measures can be used. In the present section, three measures used in this work are described. 


\subsubsection{Precision}

The precision score refers to how many individuals do really have the relevant criterion (e.g. high conscientiousness) of all individuals classified with the relevant criterion. In other words, it is defined as the proportion of individuals that are classified positive and actually are positive (true positive; TP) out of all individuals that are classified positive (true positives and false positive; TP + FP):

$$
P=T P /(T P+F P)
$$

\subsubsection{Recall}

The recall score refers to how many individuals with a relevant criterion (e.g. high conscientiousness) can be detected in the sample. It is defined as the proportion of individuals that are classified positive and are positive (TP) of all individuals that are positive (true positives and false negatives; $\mathrm{TP}+\mathrm{FN}$ ):

$$
R=T P /(T P+F N)
$$

\subsubsection{Accuracy score}

The accuracy score function computes the percentage of correct predictions. It is defined as the proportion of the number of correct classifications (true positives and true negatives; TP and TN) out of the total number of correct or incorrect classifications (true positives, true negatives, false positives and false negatives; TP, TN, FP and FN):

$$
A C C=(T P+T N) /(\mathrm{TP}+\mathrm{TN}+\mathrm{FP}+\mathrm{FN})
$$

\subsubsection{Receiver operating characteristic}

The receiver operating characteristic (ROC; Green \& Swets, 1966) curve can be used for performance visualization of a binary classifier. It visualizes the performance development of a binary classifier or predictive model depending on a variation of its discrimination threshold. To be more specific, it is a plot illustrating the true positive rate (TPR) as a function of the false positive rate (FPR) at various threshold settings. TPR is defined as the fraction of true positives out of the positives and FPR is defined as the fraction of false 
positives out of the negatives. When the ROC curve results in a diagonal line in this plot, it would indicate a performance that is as good as a random guess.

It is possible to summarize the curve information in one measure called area under the ROC curve (AUC) value. It lies somewhere between 0 and 1 and the higher it is, the better the classification performance. In case of an AUC value of 1.0, a perfect discrimination between two classes is yielded by the predictive model. If the AUC value is 0.5 , the predictive model yields the same performance as a random guess. 


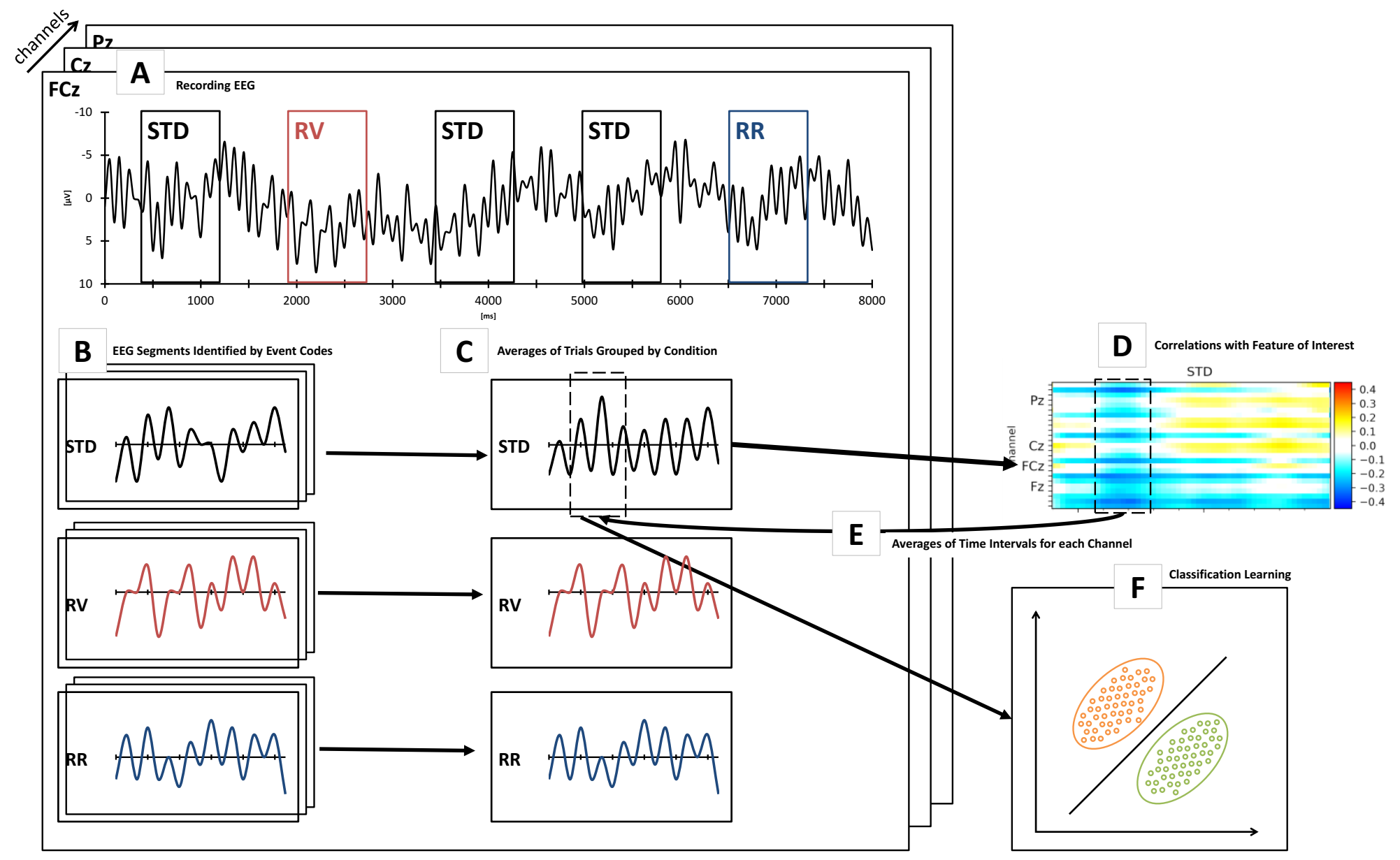

Figure 24. A schematical illustration of the classification procedure that was used for ERP data from Experiment 1 . The experiment consisted of a choice reaction task where participants during standard (STD) condition had to respond to a circle by pressing the left key and to a square by pressing the right key. In two other conditions participants had to either violate (RV) or reverse this rule (RR). EEG was recorded throughout the experiment. In this illustration only the electrode FCz is shown (A). Other electrodes are schematically indicated by different superimposed levels. The continuous signal was split into response-locked epochs (B). The raw EEG from electrode FCz is shown over a period of $8 \mathrm{~s}$, the rectangles show a $800 \mathrm{~ms}$ epoch, beginning $100 \mathrm{~ms}$ before the response. Separate averages were then computed for the three different segment types (C). After that Pearson r-values of conscientiousness and averaged response-locked ERPs were calculated for the spatio-temporal features and were displayed as color coded matrix (D). The matrix was used to determine time windows for averaging the voltage values for each electrode (E). The averaged spatio-temporal features were finally used for classification learning (F). 


\subsection{Implementation and Evaluation}

This section describes an implementation and evaluation of the previously described classification procedure. First, the classification procedure is described. Following are two subsections concerning the evaluation of the classification procedure applied to data sets obtained from the two experiments described in chapter 2 and 3. This evaluation approach aims to validate the classification procedure with two different data sets.

\subsubsection{Classification procedure}

Combining the previously described elements I propose a classification procedure which is depicted in Figure 24 and is suitable for classifying EEG data correlated with features like e.g. conscientiousness.

\subsubsection{Data acquisition}

The amplified and filtered continuous EEG signal is used as basis for the proposed procedure. I recommend performing artifact correction steps before beginning with classification learning. In this study, I used data sets of two experiments that are described in previous parts of this work (see chapter 2 and 3). For information of data preprocessing applied to the data sets see section 2.2.3 and 3.2.4. The procedure described here begins with building response-locked epochs from the continuous EEG signal. I used python 3.5 for all processing steps of the procedure and I used the python package scikit-learn (Version 0.20; Pedregosa et al., 2011) specifically for classification learning. ${ }^{1}$

\subsubsection{Feature selection and extraction}

Epochs can be grouped by different conditions when recordings of the continuous EEG signals were recorded during an experiment consisting of different conditions and the signals are containing such information. Both experiments of this work consisted of different instruction conditions and I assigned each epoch to the corresponding condition. Then the grouped epochs were averaged for each condition type and participant. In the next step, I

\footnotetext{
${ }^{1}$ I collected resources that I have programmed specifically for this application in a python library that is available on a public Git repository: https://github.com/liephat/sEEGnature
} 
calculated Pearson r-values of conscientiousness and averaged response-locked ERPs to visualize the spatio-temporal features (see section 4.2.1) and displayed them as color coded matrices. The resulting visualization served as basis for a graphical inspection and I used the matrices to determine time windows that might be good candidates for discriminating between individuals with different levels of conscientiousness. Ideally the time windows show a fairly homogenous spatial pattern of high correlations with conscientiousness. After that, I averaged the voltage values for each electrode in each participant within each selected time window and used the extracted features as basis for classification learning.

\subsubsection{Parameter optimization}

After feature extraction, I conducted a parameter optimization which is a common way in classification learning. To avoid overfitting, I conducted a 6 -fold cross validation to evaluate and find the best parameter settings. I applied this method to both LDA and LR and I used the implementations of LDA and LR of the python package scikit-learn.

\section{LDA}

In case of a high-dimensional dataset with at the same time low number of training samples, shrinkage of the covariance matrix is highly recommended. These conditions are met in the present case. Therefore, I determined the optimal shrinkage parameter by iterating the parameter from 0 to 1 in steps of 0.05 and performing a 6-fold cross-validation in each iteration step.

\section{LR}

There are different parameters which can be set: C, penalty, solver, fit_intercept. Optimal parameter settings were determined by validating all possible combinations of a set of parameters. A 6-fold cross-validation was performed for validation.

\subsubsection{Classification learning}

In the last step, I let classification models train with parameters found to be optimal by parameter optimization. 


\subsubsection{Evaluation using data from Experiment 1}

I used data acquired from Experiment 1 for evaluation of my approach classifying conscientiousness (for information about task design see section 2.2.3; for information about EEG recordings and data processing see section 2.2.4). I used averaged epochs for each participant and within-subject condition as data basis for the classification learning procedure. I used the same basis for inferential statistical analyses in section 2.3.

I created a visualization of separability measures by calculating Pearson-r values for each time point and channel combination as described in section 4.2.1. The visualization of the Pearson-r values of correct responses from Experiment 1 is depicted in Figure 25. Then, I calculated averages of voltage values for each participant within each marked time interval of Figure 25. These averages then served as features for classification learning. 
A

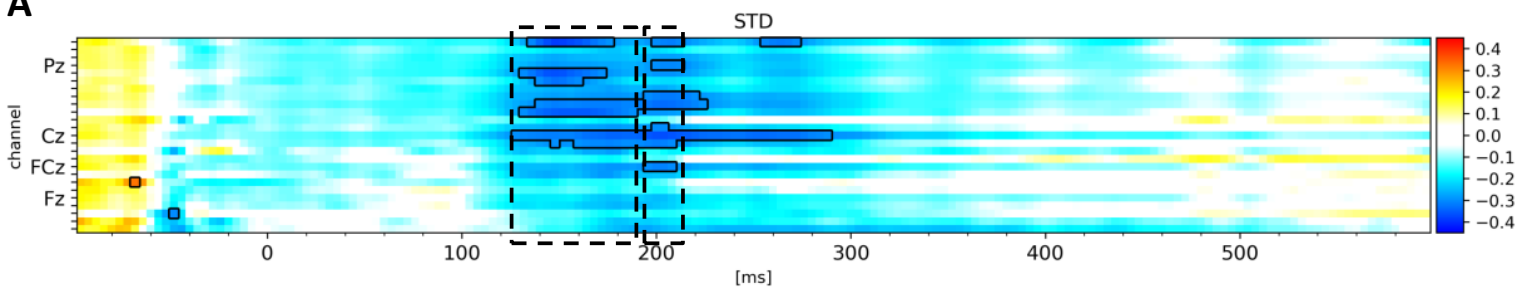

B

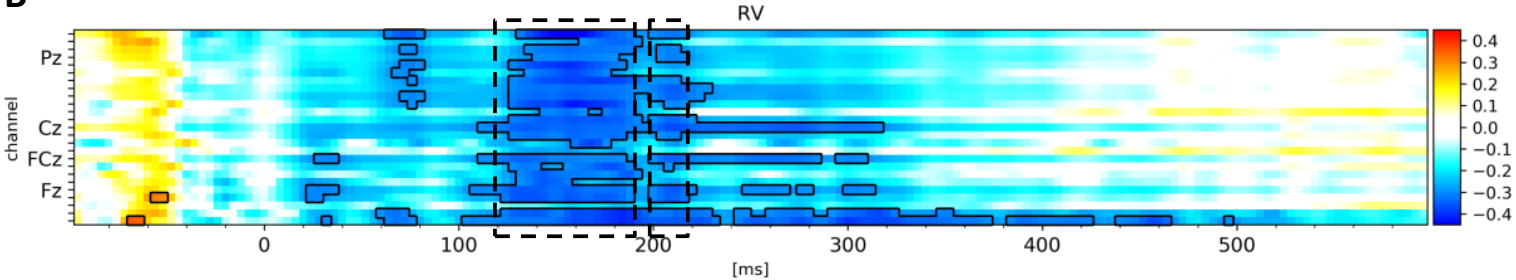

C

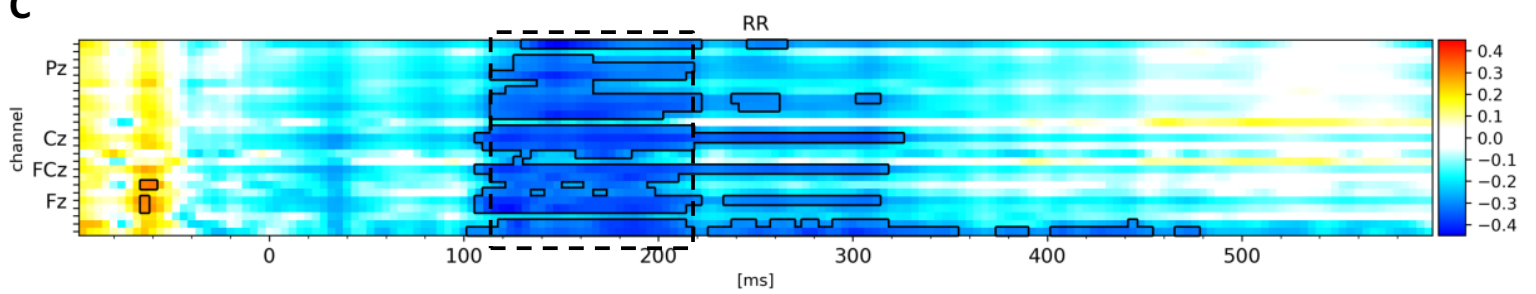

Figure 25. Visualization of the r-matrices. Pearson r-values of conscientiousness and averaged response-locked ERPs were calculated for the spatio-temporal features and displayed as a color coded matrix for each instruction condition (A: Standard, B: Rule violation, C: Rule reversal) of Experiment 1. Time intervals that are indicated by a dashed line were selected by application of a heuristic that aims to ensure having accumulated high $r$-values and an approximately constant spatial pattern of r-values in each time interval. The areas that are bounded by a continuous line indicate significant $r$-values $(p<.05)$ and help to identify suitable time intervals for classification learning.

The optimal parameter settings were determined for classification method LDA and LR as well as each instruction condition (STD, RV and RR) in Experiment 1. They are found in Table 12. 
Table 12. Results of parameter optimization for LDA and LR using data of each condition of Experiment 1.

\begin{tabular}{lccc}
\hline Parameter & Standard & Rule violation & Rule reversal \\
\hline LDA & lsqr & lsqr & 1 sqr \\
solver & 0.2 & 1.0 & 0.9 \\
shrinkage & & & \\
LR & 10 & 10 & 0.1 \\
C & True & True & True \\
fit_intercept & 11 & 11 & 11 \\
penalty & liblinear & liblinear & liblinear \\
solver & & & \\
\hline
\end{tabular}

Performance results are depicted as accuracy values in Figure 26 and as ROC curves in Figure 27. Precision and recall values are provided in Table 13. Accuracy and AUC values were compared utilizing a repeated measures ANOVA with factors iteration (10 steps) and classifier (LDA vs. LR). All effects remained non-significant, $F \mathrm{~s}<3.62, p \mathrm{~s}>.197$ and $F \mathrm{~s}<7.79, p \mathrm{~s}>.108$, respectively. 


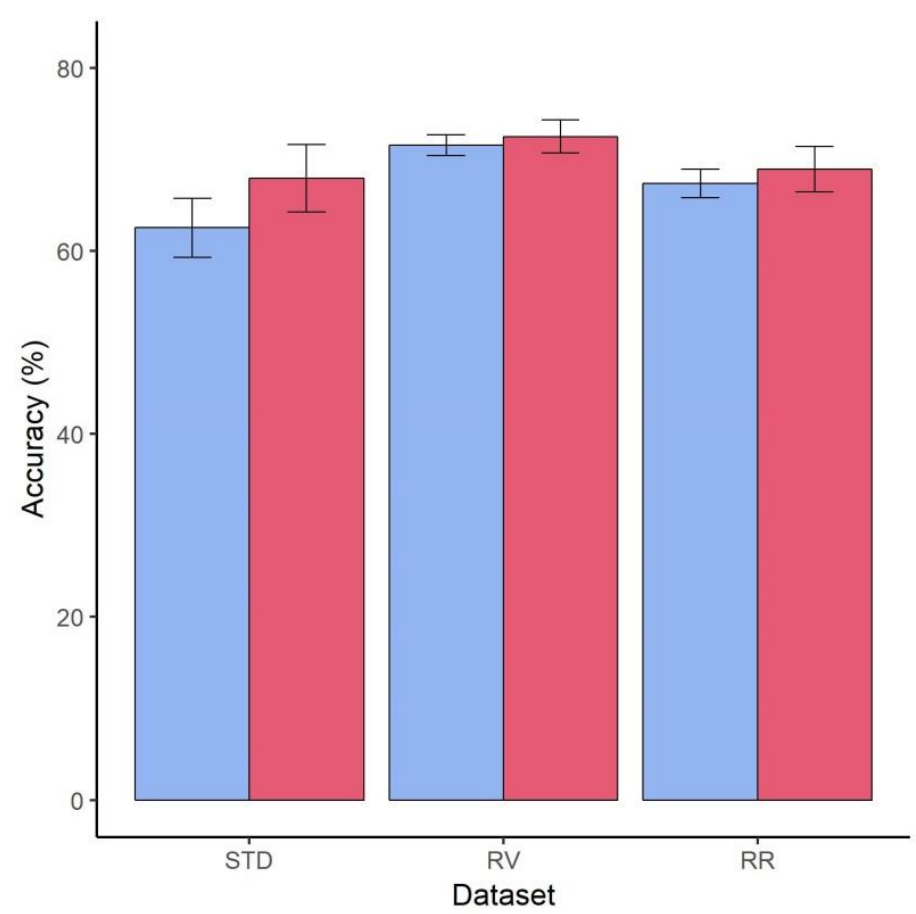

Figure 26. Performance as mean accuracy of linear discriminant analysis (LDA) with shrinkage of the covariance matrix and logistic regression (LR) for each instruction condition standard (STD), rule violation (RV) and rule reversal (RR) of Experiment 1. Mean accuracy values are aggregated for 10 iterations of 6 -fold cross validations. Error bars indicate the standard deviation. 

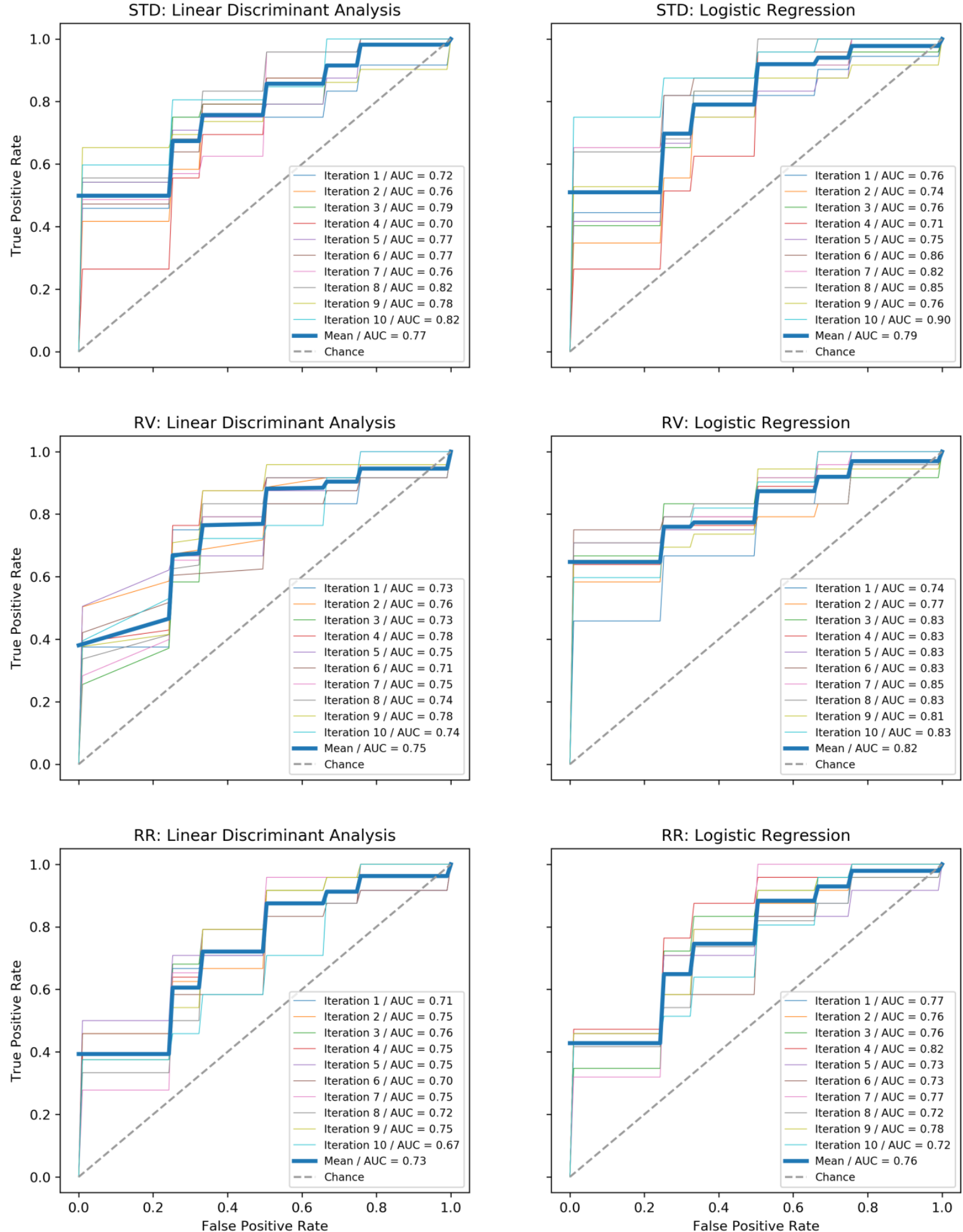

Figure 27. Performance as receiver operating characteristics (ROCs) of linear discriminant analysis (LDA) with shrinkage of the covariance matrix and logistic regression (LR) for each instruction condition standard (STD), rule violation (RV) and rule reversal (RR) of Experiment 1. ROC curves are displayed and area under the curve (AUC) values are calculated for each iteration step. 
Table 13. Classification results of linear discriminant analysis and logistic regression based on averaged spatio-temporal features drawn from the visualized r-matrices and applied to the dataset of Experiment 1.

\begin{tabular}{lcccc}
\hline & \multicolumn{2}{c}{ Linear discriminant analysis } & \multicolumn{2}{c}{ Logistic regression } \\
\hline Condition & $\begin{array}{c}\text { Precision } \\
\text { Mean }(S D)\end{array}$ & $\begin{array}{c}\text { Recall } \\
\text { Mean (SD) }\end{array}$ & $\begin{array}{c}\text { Precision } \\
\text { Mean (SD) }\end{array}$ & $\begin{array}{c}\text { Recall } \\
\text { Mean (SD) }\end{array}$ \\
$\begin{array}{l}\text { Standard } \\
\text { Low-CS }\end{array}$ & $0.64(.06)$ & $0.54(.05)$ & $0.7(.07)$ & $0.56(.04)$ \\
$\quad$ High-CS & $0.61(.04)$ & $0.69(.05)$ & $0.7(.08)$ & $0.69(.05)$ \\
Rule violation & & & & \\
$\quad$ Low-CS & $0.73(.07)$ & $0.70(.03)$ & $0.71(.07)$ & $0.72(.08)$ \\
High-CS & $0.73(.06)$ & $0.76(.05)$ & $0.75(.10)$ & $0.71(.05)$ \\
Rule reversal & & & & \\
Low-CS & $0.69(.04)$ & $0.65(.03)$ & $0.70(.04)$ & $0.67(.06)$ \\
High-CS & $0.65(.06)$ & $0.67(.04)$ & $0.71(.08)$ & $0.74(.08)$ \\
\hline
\end{tabular}

\subsubsection{Evaluation using data from Experiment 2}

I used data acquired from Experiment 2 for evaluation of my approach classifying conscientiousness (for information about task design see section 3.2.3; for information about EEG recordings and data processing see section 3.2.4). I used averaged epochs for each participant and within-subject condition as data basis for classification learning procedure. I used the same basis for inferential statistical analyses in section 3.3.

I created a visualization of separability measures by calculating Pearson-r values for each time point and channel combination as described in section 4.2.1. The visualization of the Pearson-r values of correct responses from Experiment 2 is depicted in Figure 28. Then, I calculated averages of voltage values for each participant within each marked time interval of Figure 28. These averages then served as features for classification learning. 
A

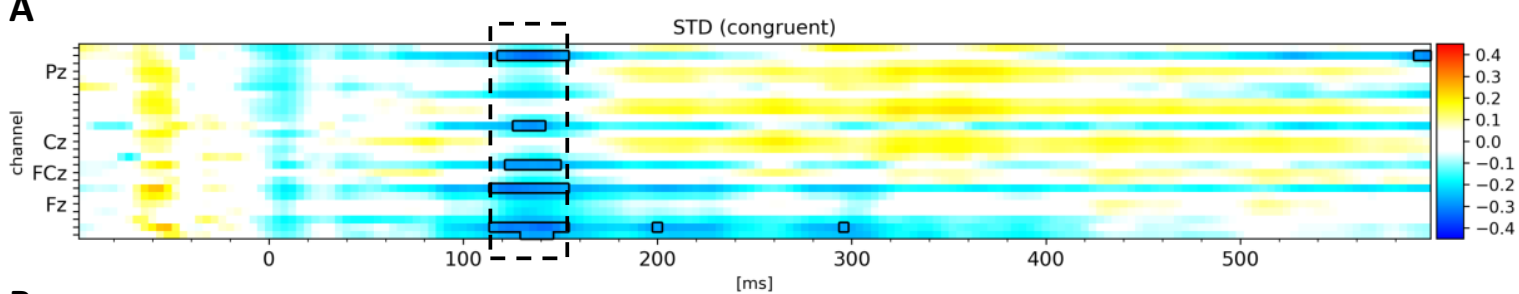

B

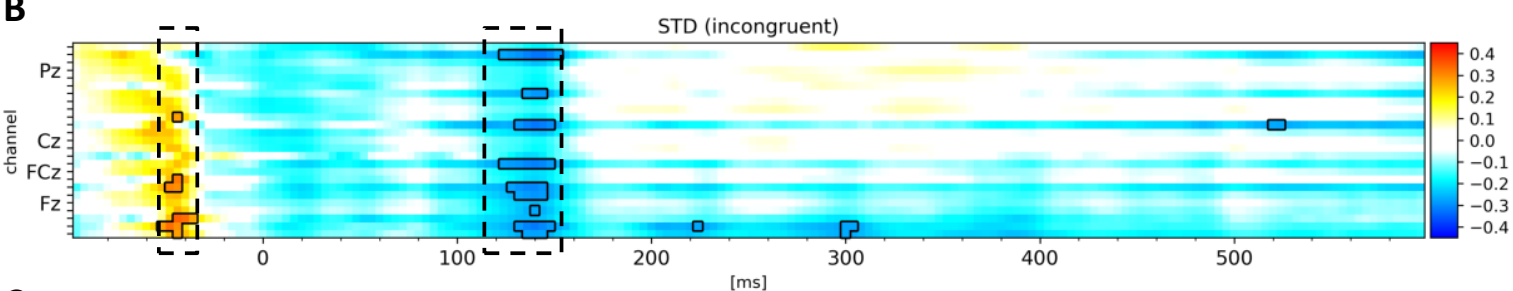

C

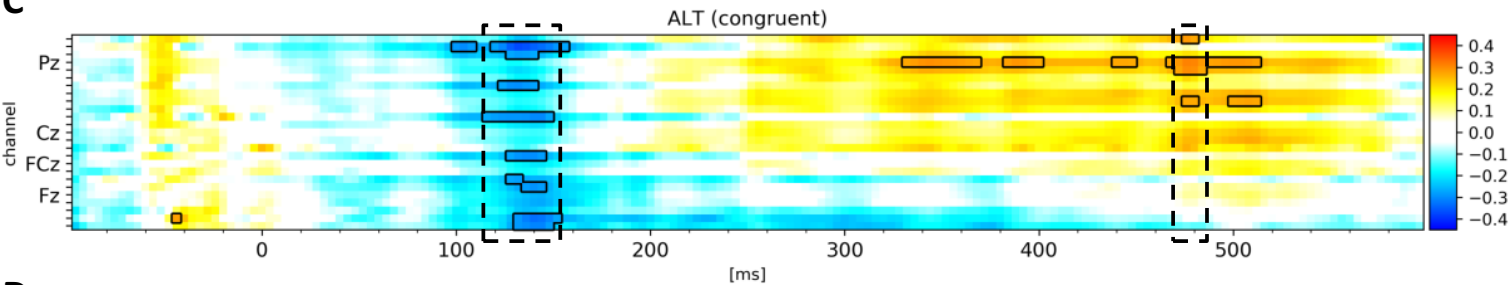

D

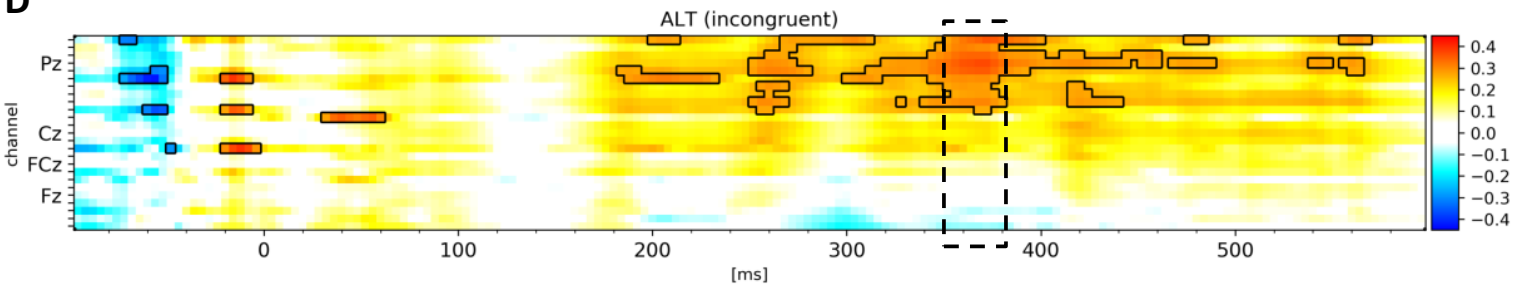

Figure 28. Visualization of the r-matrices. Pearson r-values of conscientiousness and averaged response-locked ERPs were calculated for the spatio-temporal features and were displayed as a color coded matrix for each combination of instruction and congruency condition (A: Standard congruent, B: Standard incongruent, C: Alternative instruction congruent, D: Alternative instruction incongruent) of Experiment 2 (Note: Rule violation and rule reversal instruction condition were taken together because there were no significant differences between both conditions). Time intervals that are indicated by a dashed line were selected by application of a heuristic that aims at ensuring to have accumulated high r-values and an approximately constant spatial pattern of $\mathrm{r}$-values in each time interval. The areas that are bounded by a continuous line indicate significant r-values $(p<.05)$ and help to identify suitable time intervals for classification learning.

The optimal parameter settings were determined for classification method LDA and LR as well as each instruction condition (STD congruent, STD incongruent, ALT congruent and ALT incongruent) in Experiment 2. They are found in Table 14. 
Table 14. Results of parameter optimization for linear discrimant analysis (LDA) and logistic regression (LR) using data of each condition of Experiment 2.

\begin{tabular}{lcccc}
\hline Condition & \multicolumn{2}{c}{ STD } & \multicolumn{2}{c}{ ALT } \\
\hline Congruency & congruent & incongruent & congruent & incongruent \\
\hline LDA & & & & \\
solver & 1 sqr & 1 sqr & 1 sqr & 1 sqr \\
shrinkage & 0.25 & 0.55 & 1.0 & 0.5 \\
LR & & & & \\
C & 0.1 & 0.1 & 0.1 & 1000 \\
fit_intercept & True & True & True & True \\
penalty & 11 & 11 & 11 & 11 \\
solver & liblinear & liblinear & liblinear & liblinear \\
\hline Note. STD $=$ standard instruction, ALT $=$ alternative instruction & &
\end{tabular}

Performance metrics are depicted as accuracy values in Figure 29 and as ROC curves in Figure 30. Precision and recall values are provided in Table 15. Accuracy and AUC values were compared utilizing a repeated measures ANOVA with factors iteration (10 steps) and classifier (LDA vs. LR). All effects remained non-significant, $F \mathrm{~s}<7.04, p \mathrm{~s}>.077$ and $F_{\mathrm{s}}<1.70, p \mathrm{~s}>.283$, respectively. 


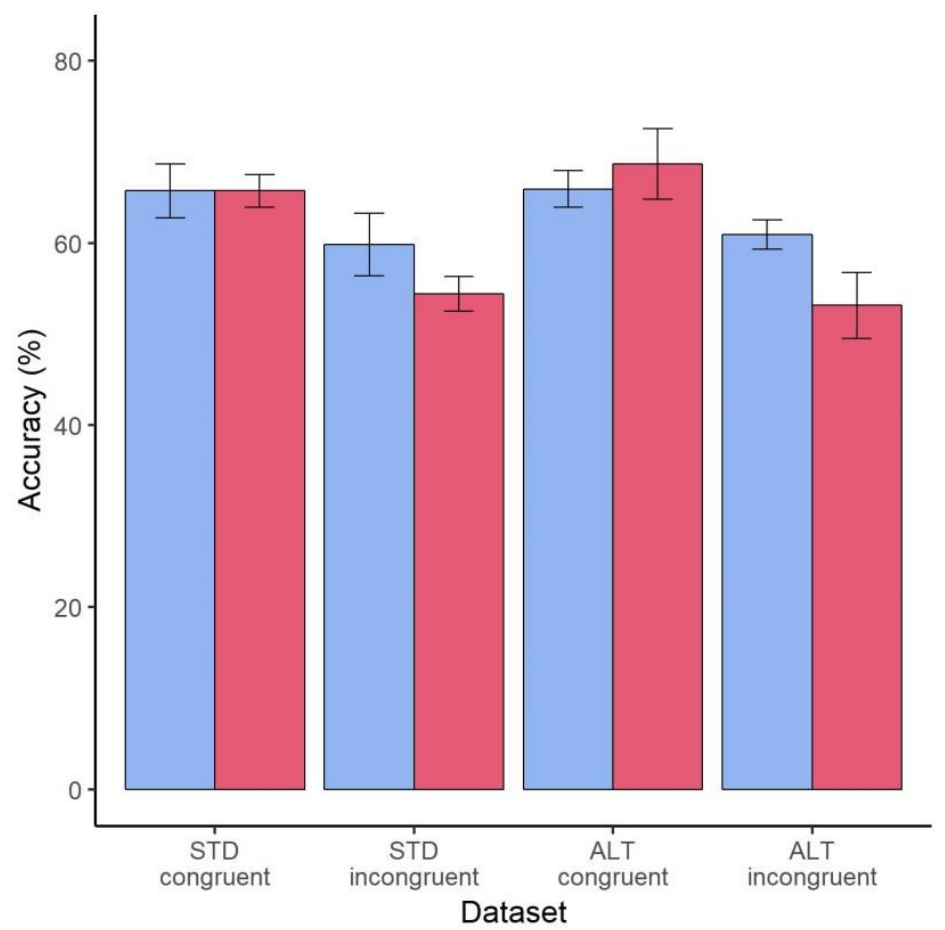

Figure 29. Performance of linear discriminant analysis (LDA) with shrinkage of the covariance matrix and logistic regression (LR) for each combination of instruction condition (standard, STD; alternative instruction, ALT) and congruency condition (congruent, incongruent) of Experiment 2. Mean accuracy values are aggregated for 10 iterations of 6-fold cross validations. Error bars indicate the standard deviation. 

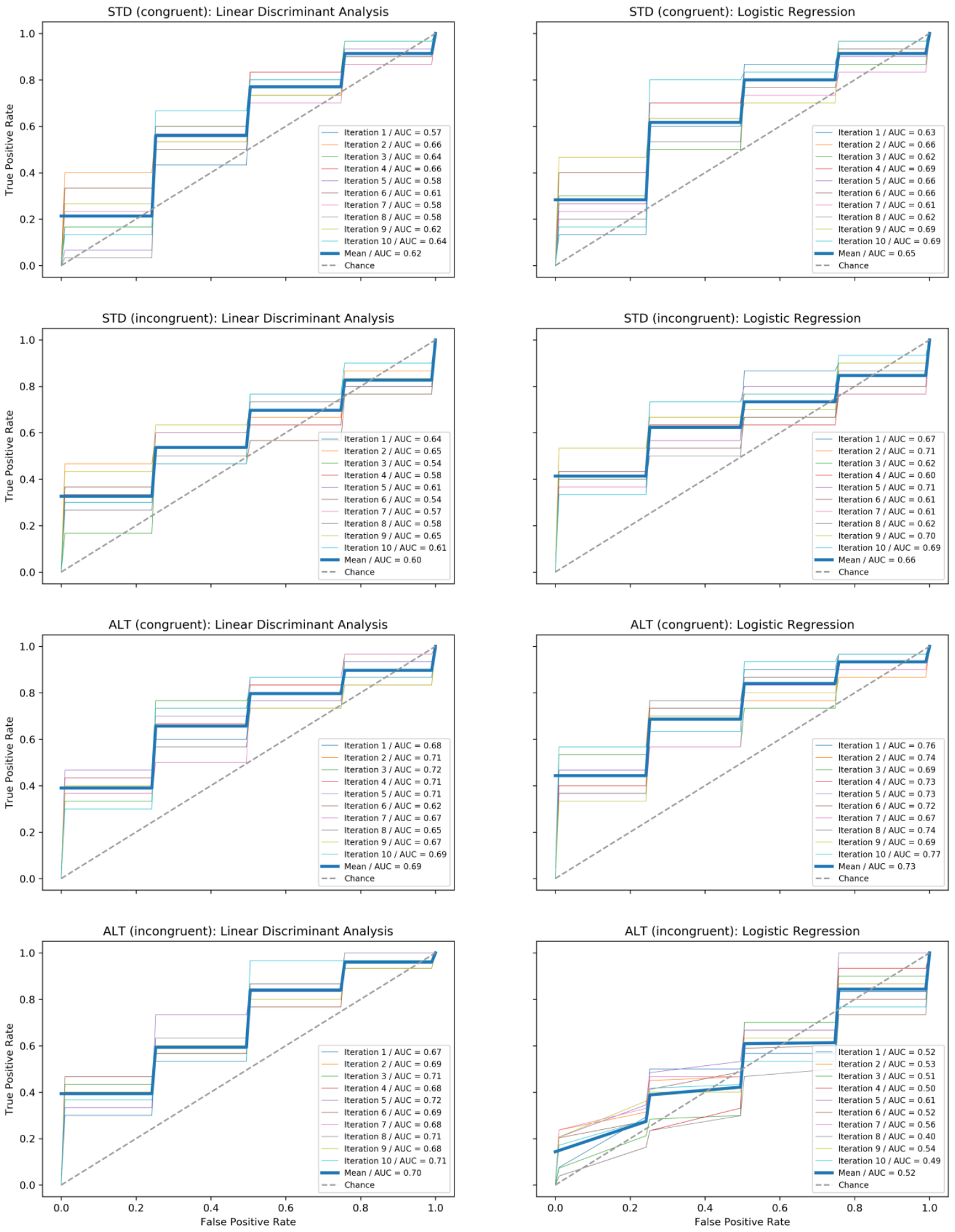

Figure 30. Performance as receiver operating characteristics (ROCs) of linear discriminant analysis (LDA) with shrinkage of the covariance matrix and logistic regression (LR) for each combination of instruction condition (standard, STD; alternative instruction, ALT) and congruency condition (congruent, incongruent) of Experiment 2. ROC curves are displayed and area under the curve (AUC) values are calculated for each iteration step. 
Table 15. Classification results of linear discriminant analysis and logistic regression based on averaged spatio-temporal features drawn from the visualized r-matrices and applied to the dataset of Experiment 2.

\begin{tabular}{lcccc}
\hline & \multicolumn{2}{c}{ Linear discriminant analysis } & \multicolumn{2}{c}{ Logistic regression } \\
\hline Condition & $\begin{array}{c}\text { Precision } \\
\text { Mean (SD) }\end{array}$ & $\begin{array}{c}\text { Recall } \\
\text { Mean (SD) }\end{array}$ & $\begin{array}{c}\text { Precision } \\
\text { Mean (SD) }\end{array}$ & $\begin{array}{c}\text { Recall } \\
\text { Mean (SD) }\end{array}$ \\
$\begin{array}{l}\text { Standard (congruent) } \\
\text { Low-CS }\end{array}$ & $0.64(0.5)$ & $0.62(.04)$ & $0.60(.08)$ & $0.49(.03)$ \\
$\quad$ High-CS & $0.71(.06)$ & $0.72(.03)$ & $0.69(.02)$ & $0.8(.02)$ \\
Standard (incongruent) & & & & \\
$\quad$ Low-CS & $0.59(.07)$ & $0.66(.06)$ & $0.46(.04)$ & $0.51(.05)$ \\
$\quad$ High-CS & $0.74(.06)$ & $0.66(.03)$ & $0.56(.07)$ & $0.55(.05)$ \\
Alternative (congruent) & & & & $0.67(.05)$ \\
$\quad$ Low-CS & $0.59(.05)$ & $0.54(.04)$ & $0.62(.07)$ & $0.69(.05)$ \\
High-CS & $0.68(.05)$ & $0.72(.03)$ & $0.76(.02)$ & \\
Alternative (incongruent) & & & & $0.45(.05)$ \\
$\quad$ Low-CS & $0.53(.06)$ & $0.53(.03)$ & $0.44(.07)$ & $0.57(.05)$ \\
$\quad$ High-CS & $0.65(.03)$ & $0.67(.03)$ & $0.54(.08)$ & \\
\hline Note. CS = conscientiousness, SD $=$ standard deviation. & & &
\end{tabular}




\subsection{Discussion}

The classification of conscientiousness in this part pursued two main goals. First, it should research the question whether there is further evidence for individual differences in the response-locked ERP associated with conscientiousness when applied alternative analysis methods. Second, it should explore potential applications of machine learning methods for detecting an electrophysiological signature of conscientiousness.

\subsubsection{Comparing results with former analyses}

Both methods LDA and LR were able to discriminate between individuals with low and high values of conscientiousness on the basis of ERP features with above chance classification using a 6-fold cross validation. The analysis confirmed results of both Experiments described in chapter 2 and 3. Very intriguingly, a comparison of feature extraction data between Experiment 1 and 2 showed similar patterns as observed before: The results confirmed that there were components in task-related ERP that were related to conscientiousness, particularly in the time window of the Pc but also CRN. As those similar patterns showed, there was also discriminative information lying in baseline area at frontal positions which can best be seen in all conditions of Experiment 1 and in the STD condition of Experiment 2, most clearly in the STD incongruent condition. Discriminative information indicated that there were differences in ERPs of individuals scoring low and high on the conscientiousness scale before the actual response was given. Future experiments might shed light upon these systematic differences during response preparation in performance monitoring.

\subsubsection{Proposed classification procedure}

Unlike common analyses comparing several means between groups, analyses applied in this part has no multiple testing problem. The reason is that learned models are not based on data used for assessing their performance (Stahl et al., 2012). The procedure introduced here has the advantage that it provides discriminative information about spatiotemporal variables and it allows for manual feature selection for the training of the classifi- 
ers. However, the manual feature selection might also be a disadvantage at the same time. It is hard to find the optimal features or feature combinations. A solution to improve the procedure and ultimately classification performance might be an algorithm that heuristically finds a good combination.

In this procedure, it is only possible to narrow and aggregate the area where the discriminative information lies, but it is usually not possible to obtain information about the importance of each variable determined by the classifier, LDA and LR in this case. There are graphical methods that can be used to evaluate the obtained results and to identify temporal and spatial subsets of ERPs that best discriminate the classes. Blankertz and colleagues (2011) e.g. described a way how to visualize weights of a linear classifier as a spatial filter.

There are other possible feature selection methods. Feature selection method for LDA with shrinkage of high-dimensional data prior to classification analysis with the aim of increasing predictive accuracy (Ahdesmäki \& Strimmer, 2010; Zuber \& Strimmer, 2009): Ranks correlation adjusted features according to their significance for the group means (centroids). Another method is to use regularized discriminant analysis with a shrunken centroid estimator performing feature selection and classification simultaneously (Guo, 2010; Guo, Hastie, \& Tibshirani, 2007). Feature selection (e.g. by ranking as described above or by visualizing correlations between a feature and spatio-temporal data as applied in this work) is not only useful for improving prediction accuracy but it also provides a set of feature variables that are relevant to discriminate the classes of interest.

\subsubsection{Limitations}

Due to the small sample sizes in both experiments (which were however quite normal for ERP experiments), not many features were possible for classification to keep an acceptable ratio of sample size to the number of features. For instance, when defining one spatio-temporal feature during feature extraction for Experiment 1 (corresponding to one time window in the color coded matrix depicted in Figure 25) the resulting ratio is 1.61 (sample size/number of channels; 45/28). In case of defining two windows this ratio de- 
creases to .80 . These ratios are far from being optimal since LDA performs well if the ratio is large ( $>20$, Stevens, 2009). However, LDA performance benefits of high covariance between features because it utilizes the covariance matrix for linear classification (see section 4.2.2.1) and high covariance is usually given in classification of EEG data (Blankertz et al., 2011).

Another limiting factor is that only the response-locked ERPs of correct responses were used to feed the classification algorithms. Response-locked ERPs of erroneous responses or difference ERPs of correct responses and errors might also contain discriminative information as it was indicated by results of Experiment 1 (see chapter 2). However, these measures could not be included into analysis because of low overall error rates in Experiment 1 and 2 .

Although there is evidence for an association of conscientiousness and the responselocked ERP, the association is relatively weak. It would be worthwhile to find additional biomarkers that are associated with conscientiousness and might help to improve classification. This issue can be addressed by further studying associations of conscientiousness and the task-related ERP. Beside the response-locked ERP the stimulus-locked ERP could also contain biomarkers linked to conscientiousness.

\subsubsection{Future improvements}

Gaussian process (Williams \& Rasmussen, 2006) might be a solution to problems observed in this study. Gaussian process models were successfully applied to classification of ERPs that reflect neurodegenerative processes in Alzheimer's disease (Fruehwirt et al., 2017). They also show good performance compared with other methods when they are applied to EEG signals for brain computer interface studies (Zhong, Lotte, Girolami, \& Lécuyer, 2008).

Averaging ERP data might lead to a loss of important information about individual differences in task performance. Including singe trial data into analysis might help to include important information about individual differences. 


\subsubsection{Conclusion}

In this realm, the application of machine learning methods including feature selection on ERP data does not only enable the detection of conscientiousness and the development of biomarkers that can be used for diagnostic purposes of this trait. It also helps to understand how conscientiousness is linked to differences in performance monitoring and this in turn may help to understand mechanisms of performance monitoring. 


\section{General Discussion}

The present work aimed at studying the electrophysiological signature of conscientiousness that is associated with task performance. For this purpose, I conducted two experiments and proposed as well as validated an approach for the classification of conscientiousness from a series of response-locked EEG records of individuals.

In Experiment 1, I utilized a simple-choice-reaction task containing the instructions to violate and swap a previously established response rule to observe individuals with different scores on a conscientiousness scale. The response-locked ERP of both errors and correct responses were of significant interest for examining characteristics of conscientiousness. Smaller $\triangle E R N$ amplitudes with almost equal CRN and ERN amplitudes and a tendency of better task performance indicated a more intensive focus on the task and a higher motivation of responding correctly in participants scoring high on a conscientiousness scale. This notion was supported by a higher Pc amplitude directly following the CRN in less conscientious participants, indicating higher task disengagement. An association of rule violations with task performance or ERP was not observed.

In Experiment 2, an alteration of the experimental design aimed at clarifying whether there is an association of rule violations with task performance and response-locked ERPs after all. An Eriksen Flanker task was employed with similar conditions as in Experiment 1 comprising instructions to violate and reverse an established rule. An ERP pattern that was similar to the pattern observed in Experiment 1, i.e. consisting of more pronounced CRN and lower Pc amplitudes present in high conflict trials as well as a tendency to better task performance might also indicate a stronger task engagement in conscientious individuals. However, the association of conscientiousness with the CRN or the Pc was weaker in relation with results of the first experiment. Additionally, there was no measurable influence of rule violations on behavioral as well as ERP data. 
The third part focused on the investigation whether task-related ERP differences observed in both experiments may function as biomarkers for classification of conscientiousness. It also aimed at finding an approach for classifying aggregated ERPs on a subject level. It revealed that the proposed approach is suitable for analysis of biomarkers and utilizing them for detection of conscientious individuals as the classification accuracy is above chance. However, especially the classification methods have some inherent issues that could be solved in future research. Nevertheless, the approach is promising and provides additional insights into data obtained from both experiments.

\subsection{Conscientiousness and task engagement}

To date, there is a lack of comprehensive theoretical approaches of performance monitoring trying to explain the emergence of various components of the ERP following responses. Cognitive theories of the ERN are powerful. They are able to explain the phenomenon of the ERN, i.e. when and how it occurs. However, even though the functional theories (e.g. conflict monitoring accounts and reinforcement learning theory) explain the emergence of the ERN, explanations of variations in its amplitude cannot sufficiently be derived. To fill this lack and to understand the functional value of the variation in the amplitude of the ERN, it might be crucial to consider motivational aspects (Weinberg et al., 2012). In addition, current cognitive models of the ERN partly neglect the emergence of the CRN.

The present work aimed to consider motivational aspects and to focus on the CRN amplitude. Results from both experiments suggest that variations in the ERN and CRN are linked to dispositional characteristics and might contribute to improvements and extensions of existing theoretical approaches to account for these variations. Only few studies investigated associations of individual differences with the CRN amplitude. To my knowledge, no study so far has examined effects of conscientiousness on components of the ERP following correct responses.

The present work provided first evidence that there are individual differences in performance monitoring that are linked to conscientiousness and are manifested in variations 
of $\triangle \mathrm{ERN}, \mathrm{CRN}$ and Pc amplitude. Higher $\mathrm{CRN}$ amplitude as well as converging ERN and CRN amplitudes in conscientious individuals indicate that the CRN magnitude might be associated with a motivational salience of correct responses. In addition, there is an association of lower Pc amplitudes and high conscientiousness. This pattern forms a contrast to an ERP pattern that has been found to be associated with anxiety and thus emphasizes the ERP pattern associated with conscientiousness. Anxiety has been found to be associated with higher ERN magnitude and diverging ERN and CRN amplitudes manifested by larger $\triangle E R N$ amplitudes (Moser et al., 2013). This ERP pattern suggests that anxious individuals seem to be afraid of committing errors having a higher motivational salience of errors. In contrast, conscientious individuals show a focus on correct responses having a higher motivational salience of responding correctly reflected by higher $\mathrm{CRN}$ and lower $\triangle \mathrm{ERN}$ amplitudes as my results suggest. This might lead to the question what ERP pattern individuals characterized by anxiety and high conscientiousness exhibit? According to the described relationships, both ERN and CRN magnitude might be enhanced while the $\triangle E R N$ amplitude does not differ between different levels of conscientiousness when anxiety is also high pronounced. However, this assumption is rather speculative and should be investigated in future studies using carefully controlled experimental settings.

The results of the present experiments also confirmed results from Pailing and Segalowitz (2004) who found the ERN amplitude of individuals low in conscientiousness to be sensitive to motivation-related changes. The findings are also in line with results from Hill et al. (2016) who observed a sensitivity of the ERN amplitude to negative urgency at low levels of conscientiousness. There seems to be no general difference in the ERN magnitude across various levels of conscientiousness. The association seems to be rather confounded by other variables. So, there might also be confounds with the higher $\Delta \mathrm{ERN}$ amplitudes observed in individuals with low conscientiousness like negative urgency (Hill et al., 2016) or anxiety (Moser et al., 2013). Future research should integrate measures for these factors into experimental settings since I did not control for these factors.

Nonetheless, the association of conscientiousness on the response-locked ERP is not as clear as it should be. The already fragile link between conscientiousness and the CRN or Pc amplitude could be interfered by adding complex experimental manipulations. 
Together with implications of recent research on individual differences in ERN and CRN (for a review see Weinberg et al., 2012), implications of the present studies might help to sharpen recent functional approaches of the ERN by considering the meaning of variations in $\mathrm{ERN}$ and $\mathrm{CRN}$.

From a more distant perspective, the observed relationship of conscientiousness with the task-related ERP may complement researching the physiological bases of personality traits. On the one hand, it illuminates conscientiousness which is a personality trait that was neglected before in this kind of research. When regarding the Five Factor Model, only extraversion and neuroticism received considerable attention in recent research on their physiological bases. On the other hand, this work suggests some methodological ideas that might help to approach the physiological basis of conscientiousness. In particular, it should be obvious to observe the EEG of conscientious individuals while they perform tasks since conscientiousness is a personality factor that might be associated with behavioral differences during task fulfillment. Furthermore, the present work suggests that it might be helpful to include situational aspects like I have tried by forcing participants to violate a rule to see whether there is an interaction of the trait conscientiousness and something that one might not want to do with a firm moral conscience. From an intuitive point of view such a situational manipulation might have an impact on the behavior of a conscientious individual. However, the behavioral as well as electrophysiological results did not confirm this assumption which can be explained by multiple reasons. First of all, operationalizing rule violations by simply instructing participants to do so does not produce a real feeling of breaking a rule. The instruction by itself might have the same or equal effect as a rule causing that following the instruction feels like acting in a rule-compliant manner. The behavior is legitimized by the instructor and might therefore not cause an internal struggle which would lead to a cognitive conflict. Second, even if instructions to violate a rule would have caused a cognitive conflict, the result must not necessarily be observable as a response conflict visible as differences in ERN or CRN like a conflict monitoring account would expect. Lastly, there might actually be no interaction of conscientiousness and violating rules at all. This conclusion however should only be drawn when both previously described explanations are rejected. 


\subsection{Classification of conscientiousness using task- related ERPs}

No study so far has tried to use ERPs as biomarkers for the classification of conscientiousness. Accuracies lying above chance verify that the well-established machine learning methods LDA and LR are suitable for the classification of conscientiousness of individuals with aggregated ERPs as data basis. Data characteristics of EEG recordings consisting of high covariances between channels help LDA to yield good classification performance. In comparison to single-trial analysis (e.g. Blankertz et al., 2011) aggregated EEG recordings as they were used in this work, should even be more suitable for classification since averaging improves the signal-to-noise ratio.

The suboptimal classification performance might be due to the curse-of-dimensionality since the number of subjects in Experiment 1 and 2, and thus the number of instances for classification was very low. However, the main reason for the performance results seems to be the rather weak association of conscientiousness and the ERP records used for classification. Even though the signals would be characterized by very good signal-to-noise ratios and the ratio of sample size to the number of features would be as good as recommended (>20; Stevens, 2009), classification results would not be optimal.

The procedure is quite suitable for analysis but it is far from being a suitable instrument for diagnostic purposes. To this end, the psychometric properties of the procedure should be further investigated.

\subsection{Future research}

There are some issues that should be addressed in future research. First, it might be worthwhile to observe associations of conscientiousness and task performance in the context of other trait or state variables, since previous studies and the present experiments suggest that the association of conscientiousness and task performance is influenced by other variables. There are also other measures of conscientiousness like HEXACO model of personality structure (Ashton et al., 2004) which also provides a measure for conscien- 
tiousness. Although the HEXACO shares common elements with the FFM, it might measure other aspects of conscientiousness. Second, it would also be worthwhile to consider investigating cognitive processes preceding responses since there were some differences in pre-response time windows that were observed between participants scoring low and high on a conscientiousness scale in the present experiments. Third, both experiments failed to establish an experimental setting that produces an ecologically valid feeling of violating a rule in participants. Hence, future research should address the challenge to design experimental settings in which participants violate rules with an intrinsic motivation. Finally, both experiments also failed to achieve an error rate that is high enough to sufficiently analyze the ERN and $\triangle E R N$ amplitude. So, evoking higher error rates in participants to incorporate $\triangle E R N$ amplitude into data analysis should be another goal in future research.

\subsection{Conclusion}

The association of conscientiousness and task performance is apparently complex. I found reasonable evidence that behavioral and response-locked ERP patterns specific to conscientious individuals might associate with higher motivation of responding correctly. This result has essentially two benefits for research: on the one hand it contributes to an understanding of the biological basis of conscientiousness and on the other it gives an idea of the functional significance of the CRN.

Nonetheless, evidence indicating influences of other factors on this association is quite striking. These influences might be inherent to motivational aspects and individual difference measures. A challenge will be to identify and control moderating variables embedded in a complex interplay to further understand what the biological foundations of conscientiousness are and how they are related to the CRN. 


\section{Reference statement}

The experiment described in chapter 2 has already been published in an article with following reference:

Imhof, M. F., \& Ruesseler, J. (2019). Performance Monitoring and Correct Response Significance in Conscientious Individuals. Frontiers in Human Neuroscience, 13, 239. https://doi.org/10.3389/fnhum.2019.00239

Open-access article with copyright (C) 2019 Imhof and Rüsseler 


\section{References}

Ahdesmäki, M., \& Strimmer, K. (2010). Feature selection in omics prediction problems using cat scores and false nondiscovery rate control. The Annals of Applied Statistics, 4(1), 503-519. https://doi.org/10.1214/09-AOAS277

Allain, S., Carbonnell, L., Falkenstein, M., Burle, B., \& Vidal, F. (2004). The modulation of the Ne-like wave on correct responses foreshadows errors. Neuroscience Letters, 372(1-2), 161-166. https://doi.org/10.1016/j.neulet.2004.09.036

Allman, J. M., Hakeem, A., Erwin, J. M., Nimchinsky, E. A., \& Hof, P. R. (2001). The anterior cingulate cortex. The evolution of an interface between emotion and cognition. Annals of the New York Academy of Sciences, 935(1), 107-117. https://doi.org/10.1111/j.1749-6632.2001.tb03476.x

Ashton, M. C., Lee, K., Perugini, M., Szarota, P., De Vries, R. E., Di Blas, L., ... De Raad, B. (2004). A six-factor structure of personality-descriptive adjectives: Solutions from psycholexical studies in seven languages. Journal of Personality and Social Psychology, 86(2), 356-366. https://doi.org/10.1037/0022-3514.86.2.356

Baldwin, S. A., Larson, M. J., \& Clayson, P. E. (2015). The dependability of electrophysiological measurements of performance monitoring in a clinical sample: A generalizability and decision analysis of the ERN and Pe. Psychophysiology, 52(6), 790-800. https://doi.org/10.1111/psyp.12401

Bartholow, B. D., Pearson, M. A., Dickter, C. L., Sher, K. J., Fabiani, M., \& Gratton, G. (2005). Strategic control and medial frontal negativity: Beyond errors and response conflict. Psychophysiology, 42(1), 33-42. https://doi.org/10.1111/j.14698986.2005.00258.x

Bates, D., Mächler, M., Bolker, B., \& Walker, S. (2014). Fitting Linear Mixed-Effects Models using lme4. ArXiv Preprint. Retrieved from http://arxiv.org/pdf/1406.5823v1

Beauducel, A., Brocke, B., \& Leue, A. (2006). Energetical bases of extraversion: Effort, arousal, EEG, and performance. International Journal of Psychophysiology, 62(2), 212223. https://doi.org/10.1016/j.ijpsycho.2005.12.001

Bernstein, P. S., Scheffers, M. K., \& Coles, M. G. H. (1995). "Where did I go wrong?" A psychophysiological analysis of error detection. Journal of Experimental Psychology: Human Perception and Performance, 21(6), 1312-1322. https://doi.org/10.1037/00961523.21.6.1312

Blankertz, B., Lemm, S., Treder, M., Haufe, S., \& Müller, K.-R. (2011). Single-trial analysis and classification of ERP components--a tutorial. NeuroImage, 56(2), 814-825. https://doi.org/10.1016/j.neuroimage.2010.06.048 
Boksem, M. A.S., Tops, M., Wester, A. E., Meijman, T. F., \& Lorist, M. M. (2006). Errorrelated ERP components and individual differences in punishment and reward sensitivity. Brain Research, 1101(1), 92-101. https://doi.org/10.1016/j.brainres.2006.05.004

Boldt, A., \& Yeung, N. (2015). Shared neural markers of decision confidence and error detection. The Journal of Neuroscience, 35(8), 3478-3484. https://doi.org/10.1523/JNEUROSCI0797-14.2015

Borkenau, P., \& Ostendorf, F. (2008). NEO-FFI : NEO-Fünf-Faktoren-Inventar nach Costa und McCrae, Manual.

Botvinick, M. M., Braver, T. S., Barch, D. M., Carter, C. S., \& Cohen, J. D. (2001). Conflict monitoring and cognitive control. Psychological Review, 108(3), 624-652. https://doi.org/10.1037/0033-295X.108.3.624

Botvinick, M. M., Nystrom, L. E., Fissell, K., Carter, C. S., \& Cohen, J. D. (1999). Conflict monitoring versus selection-for-action in anterior cingulate cortex. Nature, 402(6758), 179. https://doi.org/10.1038/46035

Brázdil, M., Roman, R., Daniel, P., \& Rektor, I. (2005). Intracerebral Error-Related Negativity in a Simple Go/NoGo Task. Journal of Psychophysiology, 19(4), 244-255. https://doi.org/10.1027/0269-8803.19.4.244

Breiman, L., \& Spector, P. (1992). Submodel Selection and Evaluation in Regression. The X-Random Case. International Statistical Review / Revue Internationale De Statistique, 60(3), 291. https://doi.org/10.2307/1403680

Burle, B., Roger, C., Allain, S., Vidal, F., \& Hasbroucq, T. (2008). Error negativity does not reflect conflict: A reappraisal of conflict monitoring and anterior cingulate cortex activity. Journal of Cognitive Neuroscience, 20(9), 1637-1655. https://doi.org/10.1162/jocn.2008.20110

Bush, G., Luu, P., \& Posner, M. I. (2000). Cognitive and emotional influences in anterior cingulate cortex. Trends in Cognitive Sciences, 4(6), 215-222. https://doi.org/10.1016/S1364-6613(00)01483-2

Carbonnell, L., \& Falkenstein, M. (2006). Does the error negativity reflect the degree of response conflict? Brain Research, 1095(1), 124-130. https://doi.org/10.1016/j.brainres.2006.04.004

Carter, C. S., Braver, T. S., Barch, D. M., Botvinick, M. M., Noll, D., \& Cohen, J. D. (1998). Anterior Cingulate Cortex, Error Detection, and the Online Monitoring of Performance. Science, 280(5364), 747-749. https://doi.org/10.1126/science.280.5364.747

Carter, C. S., \& van Veen, V. (2007). Anterior cingulate cortex and conflict detection: An update of theory and data. Cognitive, Affective, \& Behavioral Neuroscience, 7(4), 367379. https://doi.org/10.3758/CABN.7.4.367

Chiu, P. H., \& Deldin, P. J. (2007). Neural Evidence for Enhanced Error Detection in Major Depressive Disorder. American Journal of Psychiatry, 164(4), 608-616. https://doi.org/10.1176/ajp.2007.164.4.608 
Claridge, G. S., Donald, J. R., \& Birchall, P. M. (1981). Drug tolerance and personality: Some implications for Eysenck's theory. Personality and Individual Differences, 2(2), 153-166. https://doi.org/10.1016/0191-8869(81)90011-8

Cloninger, C. R. (1987). A systematic method for clinical description and classification of personality variants. A proposal. Archives of General Psychiatry, 44(6), 573-588.

Cloninger, C. R., Przybeck, T. R., Svrakic, D. M., \& Wetzel, R. D. (1994). The Temperament and Character Inventory (TCI): A guide to its development and use. St. Louis, MO: Center for Psychobiolgy of Personality, Washington University.

Coles, M. G. H., Scheffers, M. K., \& Holroyd, C. B. (2001). Why is there an ERN/Ne on correct trials? Response representations, stimulus-related components, and the theory of $\begin{array}{llll}\text { error-processing. } & \text { Biological } & \text { Psychology, } & \text { 56(3), }\end{array}$ https://doi.org/10.1016/S0301-0511(01)00076-X

Costa, P. T., \& McCrae, R. (1992). NEO-PI-R and NEO-FFI professional manual. Odessa, FL: Psychological Assessment Resources.

Cureton, E. E. (1956). Rank-biserial correlation. Psychometrika, 21(3), 287-290.

De Bruijn, E. R. A., Miedl, S. F., \& Bekkering, H. (2011). How a co-actor's task affects monitoring of own errors: evidence from a social event-related potential study. Experimental Brain Research, 211(3), 397. https://doi.org/10.1007/s00221-011-2615-1

Dehaene, S., Posner, M. I., \& Tucker, D. M. (1994). Localization of a Neural System for Error Detection and Compensation. Psychological Science, 5(5), 303-305. https://doi.org/10.1111/j.1467-9280.1994.tb00630.x

Dikman, Z. V., \& Allen, J. J. B. (2000). Error monitoring during reward and avoidance learning in high- and low-socialized individuals. Psychophysiology, 37(1), 43-54.

Ditraglia, G. M., \& Polich, J. [J.] (1991). P300 and introverted/extraverted personality types. Psychophysiology, 28(2), 177-184.

Duda, R. O., Hart, P. E., \& Stork, D. G. (2001). Pattern classification (2nd ed.). New York: Wiley.

Efron, B. (1983). Estimating the Error Rate of a Prediction Rule: Improvement on CrossValidation. Journal of the American Statistical Association, 78, 316-331.

Efron, B., \& Tibshirani, R. J. (1993). An Introduction to the Bootstrap. London: Chapman \& Hall.

Endrass, T., Klawohn, J., Gruetzmann, R., Ischebeck, M., \& Kathmann, N. (2012). Response-related negativities following correct and incorrect responses: Evidence from a temporospatial principal component analysis. Psychophysiology, 49(6), 733-743. https://doi.org/10.1111/j.1469-8986.2012.01365.x

Endrass, T., Klawohn, J., Schuster, F., \& Kathmann, N. (2008). Overactive performance monitoring in obsessive-compulsive disorder: Erp evidence from correct and erroneous reactions. Neuropsychologia, 46(7), 1877-1887. https://doi.org/10.1016/j.neuropsychologia.2007.12.001 
Endrass, T., Schuermann, B., Kaufmann, C., Spielberg, R., Kniesche, R., \& Kathmann, N. (2010). Performance monitoring and error significance in patients with obsessivecompulsive disorder. Biological Psychology, 84(2), 257-263. https://doi.org/10.1016/j.biopsycho.2010.02.002

Eriksen, B. A., \& Eriksen, C. W. (1974). Effects of noise letters upon the identification of a target letter in a nonsearch task. Perception \& Psychophysics, 16(1), 143-149. https://doi.org/10.3758/BF03203267

Ethridge, P., \& Weinberg, A. (2018). Psychometric properties of neural responses to monetary and social rewards across development. International Journal of Psychophysiology : Official Journal of the International Organization of Psychophysiology, 132(Pt B), 311-322. https://doi.org/10.1016/j.ijpsycho.2018.01.011

Eysenck, H. J. (1967). The biological basis of personality. Springfield, IL: Charles Thomas.

Eysenck, H. J. (1990). Biological dimensions of personality. In L. A. Pervin (Ed.), Handbook of personality: Theory and Research (pp. 244-276). New York: The Guildford Press.

Falkenstein, M., Hohnsbein, J., Hoormann, J., \& Blanke, L. (1990). Effects of errors in choice reaction tasks on the ERP under focused and divided attention. In C. Brunia, A. Gaillard, \& A. Kok (Eds.), Psychophysiological brain research. Tilburg: Tilburg University Press.

Falkenstein, M., Hohnsbein, J., Hoormann, J., \& Blanke, L. (1991). Effects of crossmodal divided attention on late ERP components. II. Error processing in choice reaction tasks. Electroencephalography and Clinical Neurophysiology, 78(6), 447-455.

Falkenstein, M., Hoormann, J., Christ, S., \& Hohnsbein, J. (2000). ERP components on reaction errors and their functional significance: a tutorial. Biological Psychology, 51(23), 87-107. https://doi.org/10.1016/S0301-0511(99)00031-9

Fiehler, K., Ullsperger, M., \& Cramon, D. Y. von (2005). Electrophysiological correlates of error correction. Psychophysiology, 42(1), 72-82. https://doi.org/10.1111/j.14698986.2005.00265.x

Fischer, A. G., Klein, T. A., \& Ullsperger, M. (2017). Comparing the error-related negativity across groups: The impact of error- and trial-number differences. Psychophysiology, 54(7), 998-1009. https://doi.org/10.1111/psyp.12863

Ford, J. M. (1999). Schizophrenia: The broken P300 and beyond. Psychophysiology, 36(6), $667-682$.

Foti, D., Kotov, R., \& Hajcak, G. (2013). Psychometric considerations in using errorrelated brain activity as a biomarker in psychotic disorders. Journal of Abnormal Psychology, 122(3), 655. https://doi.org/10.1037/a0033666

Friedman, J. H. (1989). Regularized discriminant analysis. Journal of the American Statistical Association, 84, 165-175.

Fruehwirt, W., Zhang, P., Gerstgrasser, M., Grossegger, D., Schmidt, R., Benke, T., ... Dorffner, G. (2017). Bayesian Gaussian Process Classification from Event-Related 
Brain Potentials in Alzheimer's Disease. In A. ten Teije, C. Popow, J. H. Holmes, \& L. Sacchi (Eds.), Artificial intelligence in medicine: 16th Conference on Artificial Intelligence in Medicine, AIME 2017, Vienna, Austria, June 21-24, 2017 : Proceedings (Vol. 10259, pp. 65-75). Cham: Springer. https://doi.org/10.1007/978-3-319-59758-4_7

Ganushchak, L. Y., \& Schiller, N. O. (2008). Motivation and semantic context affect brain error-monitoring activity: An event-related brain potentials study. NeuroImage, 39(1), 395-405. https://doi.org/10.1016/j.neuroimage.2007.09.001

Geen, R. G. (1984). Preferred stimulation levels in introverts and extroverts: Effects on arousal and performance. Journal of Personality and Social Psychology, 46(6), 13031312. https://doi.org/10.1037/0022-3514.46.6.1303

Gehring, W. J., Coles, M. G. H., Meyer, D. E., \& Donchin, E. (1990). The error-related negativity: an event-related brain potential accompanying errors. Psychophysiology, $27(4), 34$.

Gehring, W. J., Goss, B., Coles, M. G. H., Meyer, D. E., \& Donchin, E. (1993). A Neural System for Error Detection and Compensation. Psychological Science, 4(6), 385-390. https://doi.org/10.1111/j.1467-9280.1993.tb00586.x

Gehring, W. J., Himle, J., \& Nisenson, L. G. (2000). Action-monitoring dysfunction in obsessive-compulsive disorder. Psychological Science, 11(1), 1-6. https://doi.org/10.1111/1467-9280.00206

Gehring, W. J., \& Knight, R. T. (2000). Prefrontal-cingulate interactions in action monitoring. Nature Neuroscience, 3(5), 516-520. https://doi.org/10.1038/74899

Goutte, C. (1997). Note on Free Lunches and Cross-Validation. Neural Computation, 9(6), 1245-1249. https://doi.org/10.1162/neco.1997.9.6.1245

Gray, J. A. (1972). The psychology of fear and stress. New York: McGraw-Hill.

Gray, J. A. (1990). Brain systems that mediate both emotion and cognition. Motivation and Emotion, 4, 269-288.

Green, M. D., \& Swets, J. A. (1966). Signal detection theory and psychophysics. Huntington, NY: Krieger.

Guo, Y. (2010). Simultaneous variable selection and class fusion for high-dimensional linear discriminant analysis. Biostatistics (Oxford, England), 11(4), 599-608. https://doi.org/10.1093/biostatistics/kxq023

Guo, Y., Hastie, T., \& Tibshirani, R. J. (2007). Regularized linear discriminant analysis and its application in microarrays. Biostatistics (Oxford, England), 8(1), 86-100. https://doi.org/10.1093/biostatistics/kxj035

Hajcak, G., \& Foti, D. (2008). Errors are aversive: Defensive motivation and the errorrelated negativity. Psychological Science, 19(2), 103-108. https://doi.org/10.1111/j.1467-9280.2008.02053.x

Hajcak, G., McDonald, N., \& Simons, R. F. (2004). Error-related psychophysiology and negative affect. Brain and Cognition, 56(2), 189-197. https://doi.org/10.1016/j.bandc.2003.11.001 
Hajcak, G., Meyer, A., \& Kotov, R. (2017). Psychometrics and the neuroscience of individual differences: Internal consistency limits between-subjects effects. Journal of Abnormal Psychology, 126(6), 823-834. https://doi.org/10.1037/abn0000274

Hajcak, G., Moser, J. S., Yeung, N., \& Simons, R. F. (2005). On the ERN and the significance of errors. Psychophysiology, 42(2), 151-160. https://doi.org/10.1111/j.14698986.2005.00270.x

Hajcak, G., Nieuwenhuis, S., Ridderinkhof, K. R.， \& Simons, R. F. (2005). Errorpreceding brain activity: Robustness, temporal dynamics, and boundary conditions. Biological Psychology, 70(2), 67-78. https://doi.org/10.1016/j.biopsycho.2004.12.001

Hajcak, G., \& Simons, R. F. (2002). Error-related brain activity in obsessive-compulsive undergraduates. Psychiatry Research, 110(1), 63-72. https://doi.org/10.1016/S01651781(02)00034-3

Harrel, F. E. (2015). Regression modeling strategies with applications to linear models, logistic and ordinal regression, and survival analysis. New York, NY: Springer.

Hill, K. E., Samuel, D. B., \& Foti, D. (2016). Contextualizing individual differences in error monitoring: Links with impulsivity, negative affect, and conscientiousness. Psychophysiology, 53(8), 1143-1153. https://doi.org/10.1111/psyp.12671

Holroyd, C. B., \& Coles, M. G. H. (2002). The neural basis of human error processing: Reinforcement learning, dopamine, and the error-related negativity. Psychological Review, 109(4), 679-709. https://doi.org/10.1037/0033-295X.109.4.679

Holroyd, C. B., Dien, J., \& Coles, M. G. H. (1998). Error-related scalp potentials elicited by hand and foot movements: evidence for an output-independent error-processing system in humans. Neuroscience Letters, 242(2), 65-68. https://doi.org/10.1016/S03043940(98)00035-4

Holroyd, C. B., \& Yeung, N. (2003). Alcohol and error processing. Trends in Neurosciences, 26(8), 402-404. https://doi.org/10.1016/S0166-2236(03)00175-9

Holroyd, C. B., Yeung, N., Coles, M. G. H., \& Cohen, J. D. (2005). A mechanism for error detection in speeded response time tasks. Journal of Experimental Psychology. General, 134(2), 163-191. https://doi.org/10.1037/0096-3445.134.2.163

Horan, W. P., Foti, D., Hajcak, G., Wynn, J. K., \& Green, M. F. (2012). Impaired neural response to internal but not external feedback in schizophrenia. Psychological Medicine, 42(8), 1637-1647. https://doi.org/10.1017/S0033291711002819

Hosseinifard, B., Moradi, M. H., \& Rostami, R. (2013). Classifying depression patients and normal subjects using machine learning techniques and nonlinear features from EEG signal. Computer Methods and Programs in Biomedicine, 109(3), 339-345. https://doi.org/10.1016/j.cmpb.2012.10.008

Hossiep, R., Paschen, M., \& Mühlhaus, O. (2003). Bochumer Inventar zur berufsbezogenen Persönlichkeitsbeurteilung (BIP). Göttingen: Hogrefe.

Jasper, H. (1958). Report of the committee on methods of clinical examination in electroencephalography. Electroencephalography and Clinical Neurophysiology, 10, 370. 
Johannes, S., Wieringa, B. M., Nager, W., Rada, D., Dengler, R., Emrich, H. M., . . . Dietrich, D. E. (2001). Discrepant target detection and action monitoring in obsessivecompulsive disorder. Psychiatry Research: Neuroimaging, 108(2), 101-110. https://doi.org/10.1016/S0925-4927(01)00117-2

Johnson, D. L., Wiebe, J. S., Gold, S. M., Anderson, N. C., Hichwa, R. D., Watkins, G. L., \& Boles-Ponto, L. L. (1999). Biological bases of extraversion: A positron emission tomographical study. American Journal of Psychiatry, 156, 252-257.

Joyce, C. A., Gorodnitsky, I. F., \& Kutas, M. (2004). Automatic removal of eye movement and blink artifacts from EEG data using blind component separation. Psychophysiology, 41(2), 313-325. https://doi.org/10.1111/j.1469-8986.2003.00141.x

Jusyte, A., Pfister, R., Mayer, S. V., Schwarz, K. A., Wirth, R., Kunde, W., \& Schönenberg, M. (2017). Smooth criminal: Convicted rule-breakers show reduced cognitive conflict during deliberate rule violations. Psychological Research, 81(5), 939-946. https://doi.org/10.1007/s00426-016-0798-6

Kałamała, P., Drożdżowicz, A., Szewczyk, J., Marzecová, A., \& Wodniecka, Z. (2018). Task strategy may contribute to performance differences between monolinguals and bilinguals in cognitive control tasks: ERP evidence. Journal of Neurolinguistics, 46, 7892. https://doi.org/10.1016/j.jneuroling.2017.12.013

Kerns, J. G., Cohen, J. D., MacDonald, A. W., Cho, R. Y., Stenger, V. A., \& Carter, C. S. (2004). Anterior cingulate conflict monitoring and adjustments in control. Science (New York, N.Y.), 303(5660), 1023-1026. https://doi.org/10.1126/science.1089910

Kierkels, J. J. M., van Boxtel, G. J. M., \& Vogten, L. L. M. (2006). A model-based objective evaluation of eye movement correction in EEG recordings. IEEE Transactions on Bio-Medical Engineering, 53(2), 246-253. https://doi.org/10.1109/TBME.2005.862533

Kim, E. Y., Iwaki, N., Uno, H., \& Fujita, T. (2005). Error-related negativity in children: Effect of an observer. Developmental Neuropsychology, 28(3), 871-883. https://doi.org/10.1207/s15326942dn2803_7

Knyazev, G. G., Slobodskaya, H. R., \& Wilson, G. D. (2002). Psychophysiological correlates of behavioural inhibition and activation. Personality and Individual Differences, 33(4), 647-660. https://doi.org/10.1016/S0191-8869(01)00180-5

Kohavi, R. (1995). A study of cross-validation and bootstrap for accuracy estimation and model selection. Proceedings of the Fourteenth International Joint Conference on Artificial Intelligence, 2(12), 1137-1143.

Kompatsiari, K., Candrian, G., \& Mueller, A. (2016). Test-retest reliability of ERP components: A short-term replication of a visual Go/NoGo task in ADHD subjects. Neuroscience Letters, 617, 166-172. https://doi.org/10.1016/j.neulet.2016.02.012

Kuznetsova, A., Brockhoff, P. B., \& Christensen, R. H. B. (2017). 1merTest Package: Tests in Linear Mixed Effects Models. Journal of Statistical Software, 82(13). https://doi.org/10.18637/jss.v082.i13

Liu, W.-h., Wang, L.-z., Shang, H.-r., Shen, Y., Li, Z., Cheung, E. F. C., \& Chan, R. C. K. (2014). The influence of anhedonia on feedback negativity in major depressive disorder. 
Neuropsychologia,

https://doi.org/10.1016/j.neuropsychologia.2013.11.023

Lotte, F., Congedo, M., Lécuyer, A., Lamarche, F., \& Arnaldi, B. (2007). A review of classification algorithms for EEG-based brain-computer interfaces. Journal of Neural Engineering, 4(2), R1-R13. https://doi.org/10.1088/1741-2560/4/2/R01

Luck, S. J. (2014). An introduction to the event-related potential technique (2nd ed.). Cambridge: The MIT Press.

Luu, P., Collins, P., \& Tucker, D. M. (2000). Mood, personality, and self-monitoring: Negative affect and emotionality in relation to frontal lobe mechanisms of error monitoring. Journal of Experimental Psychology: General, 129(1), 43-60. https://doi.org/10.1037/0096-3445.129.1.43

Luu, P., Flaisch, T., \& Tucker, D. M. (2000). Medial Frontal Cortex in Action Monitoring. The Journal of Neuroscience, 20(1), 464-469. https://doi.org/10.1523/JNEUROSCI.2001-00464.2000

Luu, P., Tucker, D. M., Derryberry, D., Reed, M., \& Poulsen, C. (2003). Electrophysiological responses to errors and feedback in the process of action regulation. Psychological Science, 14(1), 47-53. https://doi.org/10.1111/1467-9280.01417

Luu, P., Tucker, D. M., \& Makeig, S. (2004). Frontal midline theta and the error-related negativity: Neurophysiological mechanisms of action regulation. Clinical Neurophysiology, 115(8), 1821-1835. https://doi.org/10.1016/j.clinph.2004.03.031

MacDonald, A. W., Cohen, J. D., Stenger, V. A., \& Carter, C. S. (2000). Dissociating the Role of the Dorsolateral Prefrontal and Anterior Cingulate Cortex in Cognitive Control. Science, 288(5472), 1835-1838. https://doi.org/10.1126/science.288.5472.1835

Martens, H. A., \& Dardenne, P. (1998). Validation and verification of regression in small data sets. Chemometrics and Intelligent Laboratory Systems, 44(1-2), 99-121. https://doi.org/10.1016/S0169-7439(98)00167-1

Masaki, H., Falkenstein, M., Stürmer, B., Pinkpank, T., \& Sommer, W. (2007). Does the error negativity reflect response conflict strength? Evidence from a Simon task. Psychophysiology, 44(4), 579-585. https://doi.org/10.1111/j.1469-8986.2007.00522.x

Matthews, G., \& Gilliland, K. (1999). The personality theories of H.J. Eysenck and J.A. Gray: a comparative review. Personality and Individual Differences, 26(4), 583-626. https://doi.org/10.1016/S0191-8869(98)00158-5

McCrae, R., \& Costa, P. T. (1990). Personality in adulthood. New York: Guilford.

McLoughlin, G., Makeig, S., \& Tsuang, M. T. (2014). In search of biomarkers in psychiatry: Eeg-based measures of brain function. American Journal of Medical Genetics. Part B, Neuropsychiatric Genetics, 165B(2), 111-121. https://doi.org/10.1002/ajmg.b.32208

Meckler, C., Allain, S., Carbonnell, L., Hasbroucq, T., Burle, B., \& Vidal, F. (2011). Executive control and response expectancy: A Laplacian ERP study. Psychophysiology, 48(3), 303-311. https://doi.org/10.1111/j.1469-8986.2010.01077.x 
Meyer, A., Lerner, M. D., Reyes, A. D. L., Laird, R. D., \& Hajcak, G. (2017). Considering ERP difference scores as individual difference measures: Issues with subtraction and alternative approaches. Psychophysiology, 54(1), 114-122. https://doi.org/10.1111/psyp.12664

Michie, D., Spiegelhalter, D., \& Taylor, C. (1994). Machine learning, neural and statistical classification. Upper Saddle River, NJ: Ellis Horwood.

Miltner, W. H.R., Lemke, U., Weiss, T., Holroyd, C., Scheffers, M. K., \& Coles, M. G. H. (2003). Implementation of error-processing in the human anterior cingulate cortex: a source analysis of the magnetic equivalent of the error-related negativity. Biological Psychology, 64(1-2), 157-166. https://doi.org/10.1016/S0301-0511(03)00107-8

Moser, J. S., Moran, T. P., Schroder, H. S., Donnellan, M. B., \& Yeung, N. (2013). On the relationship between anxiety and error monitoring: A meta-analysis and conceptual framework. Frontiers in Human Neuroscience, 7, 466. https://doi.org/10.3389/fnhum.2013.00466

Mueller, A., Candrian, G., Grane, V. A., Kropotov, J. D., Ponomarev, V. A., \& Baschera, G.-M. (2011). Discriminating between ADHD adults and controls using independent ERP components and a support vector machine: A validation study. Nonlinear Biomedical Physics, 5, 5. https://doi.org/10.1186/1753-4631-5-5

Mueller, A., Candrian, G., Kropotov, J. D., Ponomarev, V. A., \& Baschera, G.-M. (2010). Classification of ADHD patients on the basis of independent ERP components using a machine learning system. Nonlinear Biomedical Physics, 4 Suppl 1, S1. https://doi.org/10.1186/1753-4631-4-S1-S1

Olvet, D. M., \& Hajcak, G. (2011). The error-related negativity relates to sadness following mood induction among individuals with high neuroticism. Social Cognitive and Affective Neuroscience, 7(3), 289-295. https://doi.org/10.1093/scan/nsr007

Ostendorf, F., \& Angleitner, A. (2004). NEO-Persönlichkeitsinventar nach Costa und McCrae: NEO-PI-R ; Manual. Göttingen: Hogrefe.

Overbeek, T. J.M., Nieuwenhuis, S., \& Ridderinkhof, K. R. (2005). Dissociable Components of Error Processing. Journal of Psychophysiology, 19(4), 319-329. https://doi.org/10.1027/0269-8803.19.4.319

Pailing, P. E., \& Segalowitz, S. J. (2004). The error-related negativity as a state and trait measure: Motivation, personality, and ERPs in response to errors. Psychophysiology, 4l(1), 84-95. https://doi.org/10.1111/1469-8986.00124

Pedregosa, F., Varoquaux, G., Gramfort, A., Michel, V., Thirion, B., Grisel, O., .. . Duchesnay, É. (2011). Scikit-learn: Machine Learning in Python. Journal of Machine Learning Research, 12(Oct), 2825-2830. Retrieved from http://www.jmlr.org/papers/volume12/pedregosa11a/pedregosa1 1a.pdf

Pfister, R. (2013). Breaking the rules: Cognitive conflict during deliberate rule violations. Berlin: Logos. 
Pfister, R., Wirth, R., Schwarz, K. A., Foerster, A., Steinhauser, M., \& Kunde, W. (2016). The electrophysiological signature of deliberate rule violations. Psychophysiology, 53(12), 1870-1877. https://doi.org/10.1111/psyp.12771

Pfister, R., Wirth, R., Schwarz, K. A., Steinhauser, M., \& Kunde, W. (2016). Burdens of non-conformity: Motor execution reveals cognitive conflict during deliberate rule violations. Cognition, 147, 93-99. https://doi.org/10.1016/j.cognition.2015.11.009

Pizzagalli, D. A., Peccoralo, L. A., Davidson, R. J., \& Cohen, J. D. (2006). Resting anterior cingulate activity and abnormal responses to errors in subjects with elevated depressive symptoms: A 128-channel EEG study. Human Brain Mapping, 27(3), 185-201. https://doi.org/10.1002/hbm.20172

Pohar, M. (2004). Comparison of logistic regression and linear discriminant analysis: a simulation study. Metodoloski zvezki, 1(1), 143.

Polich, J. [John], \& Martin, S. (1992). P300, cognitive capability, and personality: A correlational study of university undergraduates. Personality and Individual Differences, 13(5), 533-543. https://doi.org/10.1016/0191-8869(92)90194-T

Ridderinkhof, K. R., Nieuwenhuis, S., \& Bashore, T. R. (2003). Errors are foreshadowed in brain potentials associated with action monitoring in cingulate cortex in humans. Neuroscience Letters, 348(1), 1-4. https://doi.org/10.1016/S0304-3940(03)00566-4

Ridderinkhof, K. R., Ullsperger, M., Crone, E. A., \& Nieuwenhuis, S. (2004). The role of the medial frontal cortex in cognitive control. Science (New York, N.Y.), 306(5695), 443-447. https://doi.org/10.1126/science.1100301

Riesel, A., Endrass, T., Kaufmann, C., \& Kathmann, N. (2011). Overactive error-related brain activity as a candidate endophenotype for obsessive-compulsive disorder: Evidence from unaffected first-degree relatives. The American Journal of Psychiatry, 168(3), 317-324. https://doi.org/10.1176/appi.ajp.2010.10030416

Riesel, A., Weinberg, A., Endrass, T., Kathmann, N., \& Hajcak, G. (2012). Punishment has a lasting impact on error-related brain activity. Psychophysiology, 49(2), 239-247. https://doi.org/10.1111/j.1469-8986.2011.01298.x

Rodríguez, J. D., Pérez, A., \& Lozano, J. A. (2010). Sensitivity analysis of kappa-fold cross validation in prediction error estimation. IEEE Transactions on Pattern Analysis and Machine Intelligence, 32(3), 569-575. https://doi.org/10.1109/TPAMI.2009.187

Roger, C., Bénar, C. G., Vidal, F., Hasbroucq, T., \& Burle, B. (2010). Rostral Cingulate Zone and correct response monitoring: Ica and source localization evidences for the unicity of correct- and error-negativities. NeuroImage, 51(1), 391-403. https://doi.org/10.1016/j.neuroimage.2010.02.005

Ruch, W. (1992). Pavlov's types of nervous system, Eysenck's typology and the Hippocrates-Galen temperaments: An empirical examination of the asserted correspondence of three temperament typologies. Personality and Individual Differences, 13(12), 12591271. https://doi.org/10.1016/0191-8869(92)90168-O

Ruchsow, M., Grön, G., Reuter, K., Spitzer, M., Hermle, L., \& Kiefer, M. (2005). ErrorRelated Brain Activity in Patients with Obsessive- Compulsive Disorder and in Healthy 
Controls. Journal of Psychophysiology, 19(4), 298-304. https://doi.org/10.1027/02698803.19.4.298

Rüsseler, J., Kuhlicke, D., \& Münte, T. F. (2003). Human error monitoring during implicit and explicit learning of a sensorimotor sequence. Neuroscience Research, 47(2), 233240. https://doi.org/10.1016/S0168-0102(03)00212-8

Scheffers, M. K., Coles, M. G. H., Bernstein, P., Gehring, W. J., \& Donchin, E. (1996). Event-related brain potentials and error-related processing: An analysis of incorrect responses to go and no-go stimuli. Psychophysiology, 33(1), 42-53. https://doi.org/10.1111/j.1469-8986.1996.tb02107.x

Simons, R. F. (2010). The way of our errors: Theme and variations. Psychophysiology, 47(1), 1-14. https://doi.org/10.1111/j.1469-8986.2009.00929.x

Stahl, D., Pickles, A., Elsabbagh, M., \& Johnson, M. H. (2012). Novel machine learning methods for ERP analysis: A validation from research on infants at risk for autism. Developmental Neuropsychology, 37(3), 274-298. https://doi.org/10.1080/87565641.2011.650808

Steinhauser, M., \& Yeung, N. (2010). Decision processes in human performance monitoring. The Journal of Neuroscience, 30(46), 15643-15653. https://doi.org/10.1523/JNEUROSCI.1899-10.2010

Stelmack, R. M. (1990). Biological Bases of Extraversion Psychophysiological Evidence. Journal of Personality, 58(1), 293-311. https://doi.org/10.1111/j.14676494.1990.tb00917.x

Stemmer, B., Witzke, W., \& Schönle, P. W. (2001). Losing the error related negativity in the EEG of human subjects: an indicator for willed action. Neuroscience Letters, 308(1), 60-62. https://doi.org/10.1016/S0304-3940(01)01974-7

Stevens, J. P. (2009). Applied multivariate statistics for the social sciences (5th ed.). New York, NY: Routledge Academic.

Strelau, J., Angleitner, A., Bantelmann, J., \& Ruch, W. (1990). The Strelau Temperament Inventory-revised (STI-R): Theoretical considerations and scale development. European Journal of Personality, 4(3), 209-235. https://doi.org/10.1002/per.2410040304

Student (1908). The probable error of a mean. Biometrika, 6, 1-25.

Suchan, B., Jokisch, D., Skotara, N., \& Daum, I. (2007). Evaluation-related frontocentral negativity evoked by correct responses and errors. Behavioural Brain Research, 183(2), 206-212. https://doi.org/10.1016/j.bbr.2007.06.013

Swick, D., \& Turken, A. U. (2002). Dissociation between conflict detection and error monitoring in the human anterior cingulate cortex. Proceedings of the National Academy of Sciences of the United States of America, 99(25), 16354-16359. https://doi.org/10.1073/pnas.252521499

Taylor, S. F., Stern, E. R., \& Gehring, W. J. (2007). Neural systems for error monitoring: Recent findings and theoretical perspectives. The Neuroscientist : A Review Journal Bringing Neurobiology, Neurology and Psychiatry, 13(2), 160-172. https://doi.org/10.1177/1073858406298184 
Tenev, A., Markovska-Simoska, S., Kocarev, L., Pop-Jordanov, J., Müller, A., \& Candrian, G. (2014). Machine learning approach for classification of ADHD adults. International Journal of Psychophysiology, 93(1), 162-166. https://doi.org/10.1016/j.ijpsycho.2013.01.008

Trujillo, L. T., \& Allen, J. J. B. (2007). Theta EEG dynamics of the error-related negativity. Clinical Neurophysiology, 118(3), 645-668. https://doi.org/10.1016/j.clinph.2006.11.009

Turken, A. U., \& Swick, D. (2008). The effect of orbitofrontal lesions on the error-related negativity. Neuroscience Letters, 441(1), 7-10. https://doi.org/10.1016/j.neulet.2008.05.115

Ullsperger, M., \& Cramon, D. Y. von (2006). The role of intact frontostriatal circuits in error processing. Journal of Cognitive Neuroscience, 18(4), 651-664. https://doi.org/10.1162/jocn.2006.18.4.651

Van Veen, V., \& Carter, C. S. (2006). Conflict and Cognitive Control in the Brain. Current Directions in Psychological Science, 15(5), 237-240. https://doi.org/10.1111/j.14678721.2006.00443.x

Vidal, F., Burle, B., Bonnet, M., Grapperon, J., \& Hasbroucq, T. (2003). Error negativity on correct trials: a reexamination of available data. Biological Psychology, 64(3), 265282. https://doi.org/10.1016/S0301-0511(03)00097-8

Vidal, F., Hasbroucq, T., Grapperon, J., \& Bonnet, M. (2000). Is the 'error negativity' specific to errors? Biological Psychology, 51(2-3), 109-128. https://doi.org/10.1016/S0301-0511(99)00032-0

Vocat, R., Pourtois, G., \& Vuilleumier, P. (2008). Unavoidable errors: A spatio-temporal analysis of time-course and neural sources of evoked potentials associated with error processing in a speeded task. Neuropsychologia, 46(10), 2545-2555. https://doi.org/10.1016/j.neuropsychologia.2008.04.006

Weinberg, A., Olvet, D. M., \& Hajcak, G. (2010). Increased error-related brain activity in generalized anxiety disorder. Biological Psychology, 85(3), 472-480. https://doi.org/10.1016/j.biopsycho.2010.09.011

Weinberg, A., Riesel, A., \& Hajcak, G. (2012). Integrating multiple perspectives on errorrelated brain activity: the ERN as a neural indicator of trait defensive reactivity. Motivation and Emotion, 36(1), 84-100.

Williams, C. K., \& Rasmussen, C. E. (2006). Gaussian processes for machine learning. Cambridge, MA: MIT press.

Wirth, R., Pfister, R., Foerster, A., Huestegge, L., \& Kunde, W. (2016). Pushing the rules: Effects and aftereffects of deliberate rule violations. Psychological Research, 80(5), 838-852. https://doi.org/10.1007/s00426-015-0690-9

Yeung, N., Botvinick, M. M., \& Cohen, J. D. (2004). The Neural Basis of Error Detection: Conflict Monitoring and the Error-Related Negativity. Psychological Review, 111(4), 931-959. https://doi.org/10.1037/0033-295X.111.4.931 
Yeung, N., \& Nieuwenhuis, S. (2009). Dissociating response conflict and error likelihood in anterior cingulate cortex. The Journal of Neuroscience, 29(46), 14506-14510. https://doi.org/10.1523/JNEUROSCI.3615-09.2009

Yordanova, J., Falkenstein, M., Hohnsbein, J., \& Kolev, V. (2004). Parallel systems of error processing in the brain. NeuroImage, 22(2), 590-602. https://doi.org/10.1016/j.neuroimage.2004.01.040

Zhong, M., Lotte, F., Girolami, M., \& Lécuyer, A. (2008). Classifying EEG for brain computer interfaces using Gaussian processes. Pattern Recognition Letters, 29(3), 354-359. https://doi.org/10.1016/j.patrec.2007.10.009

Zuber, V., \& Strimmer, K. (2009). Gene ranking and biomarker discovery under correlation. Bioinformatics (Oxford, England), 25(20), 2700-2707. https://doi.org/10.1093/bioinformatics/btp460 